\title{
Complex Manifolds
}

\section{Research Article}

\section{Michel Méo*}

\section{A dual of the Chow transformation}

https://doi.org/10.1515/coma-2018-0011

Received March 8, 2018; accepted September 11, 2018.

Abstract: We define a dual of the Chow transformation of currents on the complex projective space. This transformation factorizes a left inverse of the Chow transformation and its composition with the Chow transformation is a right inverse of a linear differential operator. In such a way we complete the general scheme of integral geometry for the Chow transformation. On another hand we prove the existence of a well defined closed positive conormal current associated to every closed positive current on the projective space. This is a consequence of the existence of a dual current, defined on the dual projective space. This allows us to extend to the case of a closed positive current the known inversion formula for the conormal of the Chow divisor of an effective algebraic cycle.

Keywords: Complex projective space, Homogeneous space, Chow divisor, Cycle space, Radon transform, John differential equations, Closed positive current, Slicing, Dual subvariety

MSC: 14C05, 32C $30,32 \mathrm{~J} 25,53 \mathrm{C} 65,58 \mathrm{~A} 25$

\section{Introduction}

For $T$ a $(q, q)$-current on $\mathbb{P}_{N}$, we denote by $\mathcal{C}(T)$ the Chow transform of $T$, which is a $(1,1)$-current on the Grassmannian $\mathbb{G}_{q-1, N}$ of $(q-1)$-dimensional projective subspaces of $\mathbb{P}_{N}$. We define $\mathcal{C}(T)$ by integrating $T$ on the projective subspaces of $\mathbb{P}_{N}$ of dimension $q-1$ i.e. $\mathcal{C}(T)$ is obtained from $T$ by means of the double fibration

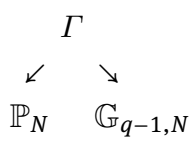

with $\Gamma \subset \mathbb{P}_{N} \times \mathbb{G}_{q-1, N}$ the incidence manifold. We prove the existence of a dual integral transform $\mathcal{C}^{*}$, defined for $(1,1)$-currents on $\mathbb{G}_{q-1, N}$, with values in

$$
\begin{array}{ll}
\left\{(q, q) \text {-currents on } \mathbb{P}_{N}\right\} & \text { if } 2 q \leq N+1, \\
\left\{(N+1-q, N+1-q) \text {-currents on } \mathbb{P}_{N}\right\} & \text { if } 2 q \geq N+1
\end{array}
$$

and satisfying the following property (see Theorem 2).

Theorem. There is a linear differential operator $\mathcal{P}$ of order $2 q-2$ with smooth coefficients, defined on

$$
\begin{array}{ll}
\left\{(q, q) \text {-currents on } \mathbb{P}_{N}\right\} & \text { if } 2 q \leq N+1, \\
\left\{(N+1-q, N+1-q) \text {-currents on } \mathbb{P}_{N}\right\} & \text { if } 2 q \geq N+1
\end{array}
$$

with values in $\left\{(q, q)\right.$-currents on $\left.\mathbb{P}_{N}\right\}$ such that $T=\left(\mathcal{P C} \mathcal{C}^{*}\right)(T)$ for all $(q, q)$-currents $T$ on $\mathbb{P}_{N}$. 
In other words, $\mathcal{C}$ is injective and a left inverse for $\mathcal{C}$ is $\mathcal{P} \mathcal{C}^{*}$. In this way we complete for the Chow transformation the general scheme of integral geometry (see [18]).

We know by [24] that the Chow transformation of currents defined on a complex projective manifold is injective and we recall here the proof by slicing of this property (see Proposition 2 and Lemma 8). An application to the problem of the approximation by algebraic cycles is also given (see subsection 6.1).

When we take for $T$ the integration current $[Z]$ associated to an algebraic cycle $Z$ in $\mathbb{P}_{N}$ of codimension $q$, the injectivity of $\mathcal{C}^{*} \mathcal{C}$ is a consequence of the following result (see Proposition 10).

Proposition. The singular support of the current $\left(\mathcal{C}^{*} \mathcal{C}\right)([Z])$ is $\operatorname{sing} \operatorname{supp}\left(\left(\mathcal{C}^{*} \mathcal{C}\right)([Z])\right)=\operatorname{supp} Z$.

The injectivity of $Z \rightarrow\left(\mathcal{C}^{*} \mathcal{C}\right)([Z])$ is also obtained by using the Schubert calculus in the Grassmannian.

When $T$ is closed positive on $\mathbb{P}_{N}$, by replacing $T$ by its conormal current $\operatorname{con}(T)$, we give a more explicit inversion formula.

In effect, $\operatorname{con}(T)$ is a well defined closed positive current on $T^{*} \mathbb{P}_{N}$ (see Proposition 9 and Theorem 4). Note that the existence of a tangent current to $T$ at a point is studied in [2, 3, 22].

By denoting by $\Phi: N^{*} \Gamma \rightarrow T^{*} \mathbb{P}_{N}$ and $\Psi: N^{*} \Gamma \rightarrow T^{*} \mathbb{G}_{q-1, N}$ the restrictions to the conormal $N^{*} \Gamma \subset$ $T^{*} \mathbb{P}_{N} \times T^{*} \mathbb{G}_{q-1, N}$ of the canonical projections, we can transform con $(T)$ by means of the double fibration

$$
\begin{array}{cc}
{ }^{N^{*}} \Gamma & \\
T^{*} \mathbb{P}_{N} & T^{*} \mathbb{G}_{q-1, N}
\end{array}
$$

obtained by considering the cotangent bundles. Then, on the conormal, the transposed map of $\Psi_{\star} \Phi^{*}$ is also a left inverse, in other words, we have the following result (see Theorem 4).

Theorem. For all closed positive $(q, q)$-currents $T$ on $\mathbb{P}_{N}$, we have $\operatorname{con}(T)=\Phi_{\star} \Psi^{*} \Psi_{\star} \Phi^{*}(\operatorname{con}(T))$.

By using the Schubert calculus, we also obtain the Skoda potential of $T$ from the Chow transform $\mathcal{C}(T)$ by means of a similar dual integral transform (see Theorem 5).

In the rest of the paper, we give new proofs of the characterization by PDE of closed $(1,1)$-currents $\Theta$ on $\mathbb{G}_{q-1, N}$ which are in the image of $\mathcal{C}$ i.e. can be written $\Theta=\mathcal{C}(T)$ with some $(q, q)$-current $T$ on $\mathbb{P}_{N}$ necessarily closed. We use homogeneous coordinates

$$
\tau:\left(\mathbb{C}^{N+1}\right)^{q} \rightarrow \mathbb{G}_{q-1, N}
$$

defined by $\tau\left(z^{0}, \ldots, z^{q-1}\right)=\operatorname{vect}\left(z^{0}, \ldots, z^{q-1}\right)$ and we write the inverse image $\tau^{\star} \Theta=\operatorname{dd}^{\mathrm{c}} U$ with some distribution $U\left(z^{0}, \ldots, z^{q-1}\right)$. We denote by $z_{m}^{l}$ the coordinates of $z^{l} \in \mathbb{C}^{N+1}$ and we consider the differential operator

$$
\partial_{I}=\operatorname{det}\left(\frac{\partial}{\partial z_{j}^{k}}\right) \underset{\substack{0 \leq k \leq q-1 \\ j \in I}}{ }
$$

for $I=\left(i_{1}, \ldots, i_{q}\right)$ with $0 \leq i_{1}<\ldots<i_{q} \leq N$.

Then $\Theta$ is a Chow transform if and only if $U$ satisfies the Gelfand-Gindikin-Graev equations

$$
0=\left(\frac{\partial^{2}}{\partial z_{m}^{l} \partial z_{m^{\prime}}^{l^{\prime}}}-\frac{\partial^{2}}{\partial z_{m^{\prime}}^{l} \partial z_{m}^{l^{\prime}}}\right) \partial_{I} \bar{\partial}_{J} U \text { and } 0=\left(\frac{\partial^{2}}{\partial \bar{z}_{m}^{l} \partial \bar{z}_{m^{\prime}}^{l^{\prime}}}-\frac{\partial^{2}}{\partial \bar{z}_{m^{\prime}}^{l} \partial \bar{z}_{m}^{l^{\prime}}}\right) \partial_{I} \bar{\partial}_{J} U
$$

for all $0 \leq l, l^{\prime} \leq q-1$, all $0 \leq m, m^{\prime} \leq N$ and all $|I|=|J|=q$.

We give a proof of this characterization by slicing (see subsection 2.2 and Proposition 4) and another proof by using the kinematic formula (see subsection 6.2). We also give a proof by using the point of view of homogeneous spaces (see section 4). Precisely we denote by $U(N+1)$ the unitary group of $\mathbb{C}^{N+1}$ and we write the double fibration in the form

$$
\begin{aligned}
& U(N+1) /(K \cap L) \\
& \begin{array}{cc}
\swarrow & \searrow \\
U(N+1) / K & U(N+1) / L
\end{array}
\end{aligned}
$$


where $K=U(1) \times U(N)$ and $L=U(q) \times U(N+1-q)$. In such a manner, we easily express the Chow transform of a smooth differential $(q-1, q-1)$-form on $\mathbb{P}_{N}$. By complexifying, we retrieve a distribution on the linear group $G L(N+1, \mathbb{C}$ ), which satisfies an equivalent system of PDE (see Theorem 3 ).

\section{Chow transformation and slicing}

We denote by $P(V)$ the projective space of lines in a complex vector space $V$ of dimension $N+1$ and $[x]$ the point of $P(V)$ associated to a nonzero vector $x$ in $V$. For $1 \leq q \leq N$, let $G(q, V)=\mathbb{G}_{q-1, N}$ be the Grassmannian of vector subspaces of $V$ of dimension $q$ and let $P(s)$ be the projective subspace of $P(V)$ associated to $s \in$ $G(q, V)$.

Let $\Gamma$ be the incidence manifold i.e. the submanifold in $P(V) \times G(q, V)$ of $([x], s)$ satisfying $s \ni x$. Then $\varphi: \Gamma \rightarrow P(V)$ and $\psi: \Gamma \rightarrow G(q, V)$ are the restrictions to $\Gamma$ of the canonical projections.

Given $T$ a current on $P(V)$ of bidimension $(p, p)$ with $p=N-q$, we set

$$
\mathcal{C}(T)=\psi_{*} \varphi^{*} T=\operatorname{pr}_{2 *}\left([\Gamma] \wedge \operatorname{pr}_{1}^{*} T\right)
$$

as being the Chow transform of $T$. Then $\mathcal{C}(T)$ is a current of bidegree $(1,1)$ on $G(q, V)$.

\subsection{Injectivity of $\mathcal{C}$}

We first recall the proof (see [24]) by slicing of the injectivity of the Chow transformation in the set of currents of bidegree $(q, q)$ on the projective space.

Proposition 1. The transformation $\mathcal{C}$ is injective in the set of currents of bidegree $(q, q)$ on $P(V)$.

Proof. This is a consequence of the injectivity of $\mathcal{C}$ in the set of $\mathcal{C}^{\infty}$ differential forms of bidegree $(q, q)$ on $P(V)$, by regularizing $T$ and $\mathcal{C}(T)$ by means of mollifier operators $J_{\varepsilon}$ on $P(V)$ and $\tilde{J}_{\varepsilon}$ on $G(q, V)$ such that

$$
\left(\tilde{J}_{\varepsilon} \circ \mathcal{C}\right)(T)=\mathcal{C}\left(J_{\varepsilon}(T)\right)
$$

and obtained from $\mathcal{C}^{\infty}$ approximations $\chi_{\varepsilon}$ of the Dirac mass at the origin of the unitary group $U(V)=U(N+1)$, for a Hermitian scalar product on $V$. We set

$$
J_{\varepsilon}(T)=\int_{g \in U(V)} \chi_{\varepsilon}(g) g^{*} T \mathrm{~d} \nu(g)
$$

with $\nu$ the Haar measure on $U(V)$, which is of total mass equal to 1 , and

$$
T_{n}=J_{\frac{1}{n}}(T) .
$$

This sequence of $\mathcal{C}^{\infty}$ differential forms on $P(V)$ weakly converges to $T$ and satisfies

$$
\mathcal{C}\left(T_{n}\right)=\int_{g \in U(V)} \chi_{\frac{1}{n}}(g) g^{*} \mathcal{C}(T) \mathrm{d} \nu(g)=\tilde{J}_{\frac{1}{n}}(\mathcal{C}(T))
$$

thus $\mathcal{C}(T)=0 \Rightarrow \mathcal{C}\left(T_{n}\right)=0$.

Now for the injectivity of $\mathcal{C}$ in the set of $\mathcal{C}^{\infty}$ differential forms of bidegree $(q, q)$ on $P(V)$, we use a slicing, to reduce to the case when the dimension of the differential form is 0 . For $T$ of $\operatorname{class} \mathcal{C}^{\infty}$, we have

$$
\mathcal{C}(T)_{\mid P\left(W^{*}\right)}=\mathcal{C}_{W}\left(T_{\mid P(W)}\right)
$$

with $P\left(W^{*}\right)=G(q, W)$ for all $W \in G(q+1, V)$. Then if $T_{\mid P(W)}=0$ for all $W$, then $T=0$. In effect, with $\pi: V \rightarrow P(V)$ the canonical map, we have

$$
\left(\pi^{*} T\right)_{x}\left(v_{1}, \ldots, v_{q}, \bar{v}_{1}, \ldots, \bar{v}_{q}\right)=\left(\pi^{*} T\right)_{\mid W}\left(x ; v_{1}, \ldots, v_{q}, \bar{v}_{1}, \ldots, \bar{v}_{q}\right)
$$




$$
=\left(\pi_{\mid W}\right)^{*}\left(T_{\mid P(W)}\right)\left(x ; v_{1}, \ldots, v_{q}, \bar{v}_{1}, \ldots, \bar{v}_{q}\right)
$$

when $W=\operatorname{vect}\left(x, v_{1}, \ldots, v_{q}\right)$, thus $\pi^{*} T=0$ since we can assume $T$ real.

In the case of the dimension 0 i.e. $q=N$, we have

$$
\mathcal{C}\left(g \omega^{N}\right) \wedge\left(\omega^{*}\right)^{N-1}=\mathcal{R}_{N-1}(g)\left(\omega^{*}\right)^{N}
$$

with $\omega$ resp. $\omega^{*}$ the Fubini-Study form on $P(V)$ resp. $P\left(V^{*}\right)$ and with $\mathcal{R}_{N-1}$ the projective hyperplane Radon transformation, defined by

$$
\mathcal{R}_{N-1}(g)(s)=\int_{P(s)} g \omega^{N-1}
$$

for $g \in \mathcal{C}^{\infty}(P(V))$ and for $s \in P\left(V^{*}\right)=G(N, V)$.

The injectivity of $\mathcal{R}_{N-1}$ is proved in [20,21]. Precisely there is a polynomial $\mathcal{P}$ of degree $N-1$ whose coefficients depend only on $N$, such that for all $g \in \mathcal{C}^{\infty}(P(V))$, we have the relation

$$
g=\mathcal{P}(\Delta) \mathcal{R}_{N-1}^{*} \mathcal{R}_{N-1} g
$$

with $\Delta$ the Laplacian on $P(V)$ associated to $\omega$ and with $\mathcal{R}_{N-1}^{*}$ the dual Radon transformation.

Note that formula (2) is still valid when $T$ is a current, thanks to the following result.

Proposition 2. For $T$ a current of bidegree $(q, q)$ on $P(V)$,

(i) a restriction current $T_{\mid P(W)}$ can be defined on $P(W)$, for all $W \in G(q+1, V)$, from a strict transform of $T$,

(ii) there is a sequence of smooth differential forms $T_{n}$ of bidegree $(q, q)$ on $P(V)$, weakly converging to $T$, such that $T_{n \mid P(W)}$ weakly converges to a current which is equal to the restriction $T_{\mid P(W)}$.

Proof. Let $\mu: M \rightarrow P(V)$ be the blowing up of $P(V)$ with center $P(W)$ and let $H \subset M$ be the exceptional divisor. According to Poly (see [26]), there is a current $\mu^{*} T$ on $M$ such that $\mu_{*} \mu^{*} T=T$. Another inverse image by $\mu$ of $T$ is of the form $\mu^{*} T+i_{\star} r$ where $i: H \rightarrow M$ is the canonical injection and $r$ is a current on $H$ such that $\nu_{\star} r=0$, with $\nu: H \rightarrow P(W)$ the fibration obtained as restriction of $\mu$ to $H$.

To define a restriction $T_{\mid P(W)}$, we are reduced to define $\left(\mu^{*} T\right)_{\mid H}$ thanks to the formula

$$
T_{\mid P(W)}=\nu_{*}\left(\left(\mu^{*} T\right)_{\mid H} \wedge \gamma\right)
$$

with $\gamma$ a closed smooth differential $(N-q-1, N-q-1)$-form on $H$ such that $\nu_{*} \gamma=1$.

But when $\lambda=\lambda\left(X_{1}, \ldots, X_{N}\right)$ is any distribution in $\left(X_{1}, \ldots, X_{N}\right) \in \mathbb{C}^{N}$, a product

$$
\begin{aligned}
\lambda \delta\left(X_{1}\right) \frac{i}{2} \mathrm{~d} X_{1} \wedge \mathrm{d} \bar{X}_{1} & =-\frac{i}{2 \pi} \lambda \bar{\partial}\left(\frac{\mathrm{d} X_{1}}{X_{1}}\right) \\
& =-\frac{i}{2 \pi}\left\{\bar{\partial}\left(\lambda \frac{\mathrm{d} X_{1}}{X_{1}}\right)-(\bar{\partial} \lambda) \wedge \frac{\mathrm{d} X_{1}}{X_{1}}\right\}
\end{aligned}
$$

is defined, by the Hörmander-Lojasiewicz division theorem. With $S=\mu^{*} T$, an intersection $S \wedge[H]$ is therefore defined, by assuming that $X_{1}=0$ is a local holomorphic equation of $H$.

Let $\sigma \in H^{0}(M, \mathcal{O}(H))$ be such that $\sigma^{-1}(0)=H$. Since $\|\sigma\|(S \wedge[H])=0$, we can write $S \wedge[H]=i_{*}\left(S_{\mid H}\right)$ with $S_{\mid H}$ some current on $H$. In such a way, a restriction $T_{\mid P(W)}$ has been defined.

Then we write this current $T_{\mid P(W)}$ as a weak limit of smooth differential $(q, q)$-forms $R_{n}$ on $P(W)$. We can extend $R_{n}$ to $P(V)$ by $T_{n}$ smooth such that $T_{n}$ weakly converges to $T$. When $T$ is closed, we can take $R_{n}$ closed, but $T_{n}$ is not necessarily both closed and smooth.

When $T$ is closed positive, the existence of almost every slice $T_{\mid P(W)}$ follows from [1, 7, 27].

Formula (3) is the projective version of the inversion formula for the hyperplane Radon transform. Let us now recall the inversion formula in the affine case, which is stated in $[8,9]$.

Let $u \in \mathcal{C}^{\infty}(V-\{0\})$ be the function defined by

$$
g([x])=\|x\|^{2 N} u(x)
$$


when $x \in V-\{0\}$ and let $\mathcal{I}_{N-1} u \in \mathcal{C}^{\infty}\left(V^{*}-\{0\}\right)$ be the function defined by

$$
\left(\mathcal{R}_{N-1} g\right)([\xi])=\|\xi\|^{2}\left(\mathcal{I}_{N-1} u\right)(\xi)
$$

when $\xi \in V^{*}-\{0\}$. First with $[P(\operatorname{Ker} \xi)]$ the current of integration on the projective hyperplane of $\mathbb{P}_{N}$ associated to $\xi$, we have

$$
\left(\mathcal{R}_{N-1} g\right)([\xi])=\int_{\mathbb{P}_{N}} g \omega^{N-1} \wedge[P(\operatorname{Ker} \xi)]
$$

and in $V=\mathbb{C}^{N+1}$, we write

$$
\pi^{*}[P(\operatorname{Ker} \xi)]=[\langle x, \xi\rangle=0]=\frac{i}{2} \delta(\langle x, \xi\rangle) \mathrm{d}\langle x, \xi\rangle \wedge \mathrm{d} \overline{\langle x, \xi\rangle} .
$$

We choose $\left(e_{0}, \ldots, e_{N}\right)$ an orthonormal basis of $V$ and take $x=e_{0}+X_{1} e_{1}+\ldots+X_{N} e_{N}$ with $X=\left(X_{1}, \ldots, X_{N}\right) \in$ $\mathbb{C}^{N}$. We use that

$$
\begin{aligned}
& g\left(\left[1, X_{1}, \ldots, X_{N}\right]\right)\left(\frac{1}{2} \mathrm{dd}^{c} \log \left(1+\|X\|^{2}\right)\right)^{N-1} \wedge \frac{i}{2} \delta\left(\langle X, v\rangle+\xi_{0}\right) \mathrm{d}\langle X, v\rangle \wedge \mathrm{d} \overline{\langle X, v\rangle} \\
& =\frac{\pi}{N}\left(\|v\|^{2}+|\langle X, v\rangle|^{2}\right) \frac{g\left(\left[1, X_{1}, \ldots, X_{N}\right]\right)}{\left(1+\|X\|^{2}\right)^{N}} \delta\left(\langle X, v\rangle+\xi_{0}\right)\left(\frac{i}{2 \pi} \partial \bar{\partial}\left(\|X\|^{2}\right)\right)^{N} \\
& =\frac{\pi}{N}\left(\|v\|^{2}+\left|\xi_{0}\right|^{2}\right) \frac{g\left(\left[1, X_{1}, \ldots, X_{N}\right]\right)}{\left(1+\|X\|^{2}\right)^{N}} \delta\left(\langle X, v\rangle+\xi_{0}\right)\left(\frac{i}{2 \pi} \partial \bar{\partial}\left(\|X\|^{2}\right)\right)^{N}
\end{aligned}
$$

where $v=\left(\xi_{1}, \ldots, \xi_{N}\right)$ and $\langle X, v\rangle=\xi_{1} X_{1}+\ldots+\xi_{N} X_{N}$. We obtain

$$
\left(\mathcal{R}_{N-1} g\right)([\xi])=\frac{\pi}{N}\|\xi\|^{2} \int_{X \in \mathbb{C}^{N}} F(X) \delta\left(\langle X, v\rangle+\xi_{0}\right)\left(\frac{i}{2 \pi} \partial \bar{\partial}\left(\|X\|^{2}\right)\right)^{N}
$$

with $F(X)=F\left(X_{1}, \ldots, X_{N}\right)=u\left(1, X_{1}, \ldots, X_{N}\right)$ for $X \in \mathbb{C}^{N}$. The affine hyperplane Radon transform of $F$ is then defined as

$$
\hat{F}(v, s)=\int_{X \in \mathbb{C}^{N}} F(X) \delta(\langle X, v\rangle-s)\left(\frac{i}{2} \partial \bar{\partial}\left(\|X\|^{2}\right)\right)^{N}
$$

for $v \in \mathbb{C}^{N} \backslash\{0\}$ and $s \in \mathbb{C}$, in such a way that

$$
\left(\mathcal{I}_{N-1} u\right)\left(\xi_{0}, \xi_{1}, \ldots, \xi_{N}\right)=\frac{1}{N \pi^{N-1}} \hat{F}\left(v,-\xi_{0}\right) .
$$

The classical inversion theorem states that there is a constant $c_{N} \neq 0$ such that

$$
F(X)=c_{N} \int_{v \in K} \frac{\partial^{2(N-1)} \hat{F}}{\partial s^{N-1} \partial \bar{s}^{N-1}}(v,\langle X, v\rangle) \Phi(v)
$$

where the differential form $\Phi(v)=\Phi\left(\xi_{1}, \ldots, \xi_{N}\right)$ is given by

$$
\begin{array}{r}
\Phi(v)=\frac{i}{4 \pi} \sum_{1 \leq k \leq N}(-1)^{k-1}\left(\xi_{k} \mathrm{~d} \xi_{1} \wedge \ldots \wedge \widehat{\mathrm{d}}_{k} \wedge \ldots \wedge \mathrm{d} \xi_{N} \wedge \mathrm{d} \bar{\xi}_{1} \wedge \ldots \wedge \mathrm{d} \bar{\xi}_{N}\right. \\
\left.+(-1)^{N} \bar{\xi}_{k} \mathrm{~d} \xi_{1} \wedge \ldots \wedge \mathrm{d} \xi_{N} \wedge \mathrm{d} \bar{\xi}_{1} \wedge \ldots \wedge \widehat{\mathrm{d} \bar{\xi}_{k}} \wedge \ldots \wedge \mathrm{d} \bar{\xi}_{N}\right)
\end{array}
$$

and where $K=\mathbb{S}^{2 N-1}=\left\{v \in \mathbb{C}^{N},\|v\|=1\right\}$.

\subsection{Determination of $\operatorname{im} \mathcal{C} \cap$ ker d}

We obtain here, by slicing and by using the affine inversion formula, the Gelfand-Gindikin-Graev equations characterizing the images by the Chow transformation of closed $(q, q)$-currents on $\mathbb{P}_{N}$. 
Let $\Theta=\mathcal{C}(T)$ be a real smooth differential $(1,1)$-form on $G(q, V)$, so which is in im $\mathcal{C}$. Then $T$ is also real. A first condition is that for all $W \in G(q+1, V)$, the restriction $\Theta_{\mid P\left(W^{*}\right)}$ of $\Theta$ to $P\left(W^{*}\right)=G(q, W)$ is closed i.e.

$$
(\mathrm{d} \Theta)_{\mid P\left(W^{*}\right)}=0 .
$$

This follows from (2), since $T_{\mid P(W)}$ is of bidegree $(q, q)$ on $P(W)$, thus closed.

On the other hand, by setting $T_{\mid P(W)}=g \omega_{\mid P(W)}^{q}$ with $g \in \mathcal{C}^{\infty}(P(W))$, we have

$$
\Theta_{\mid P\left(W^{*}\right)} \wedge\left(\omega_{W}^{*}\right)^{q-1}=\mathcal{C}_{W}\left(T_{\mid P(W)}\right) \wedge\left(\omega_{W}^{*}\right)^{q-1}=\mathcal{R}_{q-1}(g)\left(\omega_{W}^{*}\right)^{q}
$$

where $\omega_{W}^{*}$ is the Fubini-Study form on $P\left(W^{*}\right)$ and with the projective $(q-1)$-dimensional Radon transform given by

$$
\mathcal{R}_{q-1}(g)(s)=\int_{P(s)} g \omega^{q-1}
$$

for $s \in P\left(W^{*}\right)=G(q, W)$. The inverse transform $\mathcal{C}_{W}^{-1}\left(\Theta_{\mid P\left(W^{*}\right)}\right)=g \omega_{\mid P(W)}^{q}$ can be calculated by applying formula (4) since $s \in P\left(W^{*}\right)$ is a hyperplane in $W$.

We fix $[x] \in P(V)$ and we take $W=\operatorname{vect}\left(x, v_{1}, \ldots, v_{q}\right)$ for $v_{1}, \ldots, v_{q} \in V$. Then a second condition is that

$$
\begin{aligned}
& \mathcal{C}_{W}^{-1}\left(\Theta_{\mid P\left(W^{*}\right)}\right)\left([x] ; \mathrm{d} \pi_{x}\left(v_{1}\right), \ldots, \mathrm{d} \pi_{x}\left(v_{q}\right), \overline{\mathrm{d} \pi_{x}\left(v_{1}\right)}, \ldots, \overline{\mathrm{d} \pi_{x}\left(v_{q}\right)}\right) \\
= & T_{[x]}\left(\mathrm{d} \pi_{x}\left(v_{1}\right), \ldots, \mathrm{d} \pi_{x}\left(v_{q}\right), \overline{\mathrm{d} \pi_{x}\left(v_{1}\right)}, \ldots, \overline{\mathrm{d} \pi_{x}\left(v_{q}\right)}\right) \\
= & \left(\pi^{*} T\right)_{x}\left(v_{1}, \ldots, v_{q}, \bar{v}_{1}, \ldots, \bar{v}_{q}\right)
\end{aligned}
$$

is a Hermitian quadratic form of $v_{1} \wedge \ldots \wedge v_{q} \in \wedge^{q} \mathbb{C}^{N+1}=\wedge^{q} V$. We use the Plücker coordinates $v_{I}$ defined by

$$
v_{1} \wedge \ldots \wedge v_{q}=\sum_{|I|=q} v_{I} e_{I}
$$

where $e_{I}=e_{i_{1}} \wedge \ldots \wedge e_{i_{q}}$ for $I=\left(i_{1}, \ldots, i_{q}\right)$ with $0 \leq i_{1}<\ldots<i_{q} \leq N$. This second condition is therefore that

$$
\frac{\partial^{2}}{\partial v_{I} \partial \bar{v}_{J}}\left\{\mathcal{C}_{W}^{-1}\left(\Theta_{\mid P\left(W^{*}\right)}\right)\left([x] ; \mathrm{d} \pi_{x}\left(v_{1}\right), \ldots, \mathrm{d} \pi_{x}\left(v_{q}\right), \overline{\mathrm{d} \pi_{x}\left(v_{1}\right)}, \ldots, \overline{\mathrm{d} \pi_{x}\left(v_{q}\right)}\right)\right\}
$$

is independent of $v_{1} \wedge \ldots \wedge v_{q}$. Since $v_{n} \wedge v_{n^{\prime}}=\sum_{m<m^{\prime}}\left(v_{m}^{n} v_{m^{\prime}}^{n^{\prime}}-v_{m^{\prime}}^{n} v_{m}^{n^{\prime}}\right) e_{m} \wedge e_{m^{\prime}}$, we use coordinates $w$ of the type

$$
w=w_{m, m^{\prime}}^{n, n^{\prime}}=v_{m}^{n} v_{m^{\prime}}^{n^{\prime}}-v_{m^{\prime}}^{n} v_{m}^{n^{\prime}}
$$

for $1 \leq n, n^{\prime} \leq q$ and $0 \leq m, m^{\prime} \leq N$, where the $v_{m}^{n}$ are the coordinates of $v_{n}$ in the basis $e_{0}, \ldots, e_{N}$. The above condition becomes

$$
\begin{aligned}
0 & =\frac{\partial}{\partial w} \frac{\partial^{2}}{\partial v_{I} \partial \bar{v}_{J}}\left\{\mathcal{C}_{W}^{-1}\left(\Theta_{\mid P\left(W^{*}\right)}\right)\left([x] ; \mathrm{d} \pi_{x}\left(v_{1}\right), \ldots, \mathrm{d} \pi_{x}\left(v_{q}\right), \overline{\mathrm{d} \pi_{x}\left(v_{1}\right)}, \ldots, \overline{\mathrm{d} \pi_{x}\left(v_{q}\right)}\right)\right\} \\
& =\frac{\partial}{\partial \bar{W}} \frac{\partial^{2}}{\partial v_{I} \partial \bar{v}_{J}}\left\{\mathcal{C}_{W}^{-1}\left(\Theta_{\mid P\left(W^{*}\right)}\right)\left([x] ; \mathrm{d} \pi_{x}\left(v_{1}\right), \ldots, \mathrm{d} \pi_{x}\left(v_{q}\right), \overline{\mathrm{d} \pi_{x}\left(v_{1}\right)}, \ldots, \overline{\mathrm{d} \pi_{x}\left(v_{q}\right)}\right)\right\}
\end{aligned}
$$

the second equality being then automatic, since $\Theta$ is real. We use the parametrization $\lambda$ of $W$ given by

$$
\lambda\left(t_{0}, t_{1}, \ldots, t_{q}\right)=t_{0} x+\sum_{1 \leq j \leq q} t_{j} v_{j} \in W
$$

for $\left(t_{0}, t_{1}, \ldots, t_{q}\right) \in \mathbb{C}^{q+1}$. This gives rise to an isomorphism induced by $\lambda$, defined by

$$
\begin{aligned}
& \mathbb{P}_{q}^{*}=G\left(q, \mathbb{C}^{q+1}\right) \stackrel{\lambda}{\rightarrow} G(q, W)=P\left(W^{*}\right) \\
& \operatorname{vect}\left(t^{0}, \ldots, t^{q-1}\right) \rightarrow \operatorname{vect}\left(\sum_{0 \leq j \leq q} t_{j}^{k} v_{j}\right)_{0 \leq k \leq q-1}
\end{aligned}
$$

where $v_{0}=x$. With $\mathcal{C}_{\mathbb{C}^{q+1}}$ the Chow transform on $\mathbb{P}_{q}=P\left(\mathbb{C}^{q+1}\right)$, we have

$$
\lambda^{*}\left(\mathcal{C}_{W}^{-1}\left(\Theta_{\mid P\left(W^{*}\right)}\right)\right)=\mathcal{C}_{\mathbb{C}^{+1}}^{-1}\left(\lambda^{*}\left(\Theta_{\mid P\left(W^{*}\right)}\right)\right)
$$


and with $b_{0}, \ldots, b_{q}$ the canonical basis of $\mathbb{C}^{q+1}$, we have

$$
\begin{aligned}
& \mathcal{C}_{W}^{-1}\left(\Theta_{\mid P\left(W^{*}\right)}\right)\left([x] ; \mathrm{d} \pi_{x}\left(v_{1}\right), \ldots, \mathrm{d} \pi_{x}\left(v_{q}\right), \overline{\mathrm{d} \pi_{x}\left(v_{1}\right)}, \ldots, \overline{\mathrm{d} \pi_{x}\left(v_{q}\right)}\right) \\
& =\mathcal{C}_{\mathbb{C}^{q+1}}^{-1}\left(\lambda^{*}\left(\Theta_{\mid P\left(W^{*}\right)}\right)\right)\left(\left[b_{0}\right] ; b_{0}^{-1} \otimes \tilde{b}_{1}, \ldots, b_{0}^{-1} \otimes \tilde{b}_{q}, \overline{b_{0}^{-1} \otimes \tilde{b}_{1}}, \ldots, \overline{b_{0}^{-1} \otimes \tilde{b}_{q}}\right)
\end{aligned}
$$

where $\tilde{b}_{k}=\left(b_{k} \bmod \mathbb{C} b_{0}\right) \in \mathbb{C}^{q+1} / \mathbb{C} b_{0}$. Therefore the second condition is

$$
0=\mathcal{C}_{\mathbb{C}^{q+1}}^{-1}\left(\frac{\partial}{\partial w} \frac{\partial^{2}}{\partial v_{I} \partial \bar{v}_{J}} \lambda^{*}\left(\Theta_{\mid P\left(W^{*}\right)}\right)\right)\left(\left[b_{0}\right] ; b_{0}^{-1} \otimes \tilde{b}_{1}, \ldots, b_{0}^{-1} \otimes \tilde{b}_{q}, \overline{b_{0}^{-1} \otimes \tilde{b}_{1}}, \ldots, \overline{b_{0}^{-1} \otimes \tilde{b}_{q}}\right) .
$$

In effect, $\Theta_{\mid P\left(W^{*}\right)}$ is closed and we can write the closed smooth differential (1,1)-form

$$
\frac{\partial}{\partial w} \frac{\partial^{2}}{\partial v_{I} \partial \bar{v}_{J}} \lambda^{*}\left(\Theta_{\mid P\left(W^{*}\right)}\right)=\mathcal{C}_{\mathbb{C}^{q+1}}\left(f \omega_{\mathbb{C}^{q+1}}^{q}\right)
$$

with $f \in \mathcal{C}^{\infty}\left(\mathbb{P}_{q}\right)$ and with $\omega_{\mathbb{C}^{q+1}}$ the Fubini-Study form on $\mathbb{P}_{q}$. Then equation (6) reduces to

$$
\begin{aligned}
0 & =\left(f \omega_{\mathbb{C}^{q+1}}^{q}\right)\left(\left[b_{0}\right] ; b_{0}^{-1} \otimes \tilde{b}_{1}, \ldots, b_{0}^{-1} \otimes \tilde{b}_{q}, \overline{b_{0}^{-1} \otimes \tilde{b}_{1}}, \ldots, \overline{b_{0}^{-1} \otimes \tilde{b}_{q}}\right) \\
\Leftrightarrow 0 & =f\left(\left[b_{0}\right]\right) .
\end{aligned}
$$

Assume now $\Theta$ closed in $G(q, V)$ i.e. $\mathrm{d} \Theta=0$, instead of (5). Denote by $\tau: V^{q} \rightarrow G(q, V)$ the map which associates to $z=\left(z^{0}, \ldots, z^{q-1}\right)$ in $V^{q}$ the vector subspace $\tau(z)=\operatorname{vect}\left(z^{0}, \ldots, z^{q-1}\right) \in G(q, V)$. The inverse image by $\tau$ of $\Theta$ is then written as

$$
\tau^{*} \Theta=\operatorname{dd}^{\mathrm{c}} U
$$

with $U(z)=U\left(z^{0}, \ldots, z^{q-1}\right)$ some function on $V^{q}$. Since

$$
\lambda^{*}\left(\Theta_{\mid P\left(W^{*}\right)}\right)=\operatorname{dd}^{\mathrm{c}} \lambda^{*}\left(U_{\mid W^{q}}\right)=\operatorname{dd}^{\mathrm{c}}\left(U\left(\sum_{0 \leq j \leq q} t_{j}^{0} v_{j}, \ldots, \sum_{0 \leq j \leq q} t_{j}^{q-1} v_{j}\right)\right),
$$

the function $f$, which depends on $v_{1}, \ldots, v_{q}$, satisfies the relation

$$
\operatorname{dd}^{\mathrm{c}}\left\{\frac{\partial}{\partial w} \frac{\partial^{2}}{\partial v_{I} \partial \bar{v}_{J}}\left(U\left(\sum_{0 \leq j \leq q} t_{j}^{0} v_{j}, \ldots, \sum_{0 \leq j \leq q} t_{j}^{q-1} v_{j}\right)\right)\right\} \wedge\left(\omega_{\mathbb{C}^{q+1}}^{*}\right)^{q-1}=\mathcal{R}_{q-1, \mathbb{C}^{q+1}}(f)\left(\omega_{\mathbb{C}^{q+1}}^{*}\right)^{q}
$$

where $\omega_{\mathbb{C}^{q+1}}^{*}$ is the Fubini-Study form on $\mathbb{P}_{q}^{*}=G\left(q, \mathbb{C}^{q+1}\right)$ and with $\mathcal{R}_{q-1, \mathbb{C}^{q+1}}$ the hyperplane Radon transform on $\mathbb{P}_{q}$.

But by using the coordinates $v_{m}^{n}$ for $1 \leq n \leq q$ and $0 \leq m \leq N$ and by expressing the differential operators

$$
\frac{\partial}{\partial w}=\frac{\partial}{\partial w_{m, m^{\prime}}^{n, n^{\prime}}}=\frac{\partial^{2}}{\partial v_{m}^{n} \partial v_{m^{\prime}}^{n^{\prime}}}-\frac{\partial^{2}}{\partial v_{m^{\prime}}^{n} \partial v_{m}^{n^{\prime}}}
$$

and

$$
\frac{\partial}{\partial v_{I}}=\operatorname{det}\left(\frac{\partial}{\partial v_{j}^{k}}\right) \underset{\substack{1 \leq k \leq q \\ j \in I}}{ }
$$

first we have the relation

$$
\begin{array}{r}
\frac{\partial}{\partial w} \frac{\partial^{2}}{\partial v_{I} \partial \bar{v}_{J}}\left(U\left(t_{0}^{0} x+\sum_{1 \leq j \leq q} t_{j}^{0} v_{j}, \ldots, t_{0}^{q-1} x+\sum_{1 \leq j \leq q} t_{j}^{q-1} v_{j}\right)\right)=\left|\operatorname{det}\left(t_{j}^{k}\right)_{\substack{0 \leq k \leq q-1 \\
1 \leq j \leq q}}\right|^{2} \\
\cdot \sum_{0 \leq l, l^{\prime} \leq q-1} t_{n}^{l} t_{n^{\prime}}^{l^{\prime}}\left(\left(\frac{\partial^{2}}{\partial z_{m}^{l} \partial z_{m^{\prime}}^{l^{\prime}}}-\frac{\partial^{2}}{\partial z_{m^{\prime}}^{l} \partial z_{m}^{l^{\prime}}}\right) \partial_{I} \bar{\partial}_{J} U\right)\left(t_{0}^{0} x+\sum_{1 \leq j \leq q} t_{j}^{0} v_{j}, \ldots, t_{0}^{q-1} x+\sum_{1 \leq j \leq q} t_{j}^{q-1} v_{j}\right)
\end{array}
$$

where

$$
\partial_{I}=\operatorname{det}\left(\frac{\partial}{\partial z_{j}^{k}}\right) \underset{\substack{0 \leq k \leq q-1 \\ j \in I}}{ } .
$$


Then by calculating $f\left(\left[b_{0}\right]\right)$ by means of the formulas (4) and (7), we conclude that the condition (6) is equivalent to the Gelfand-Gindikin-Graev equations

$$
0=\left(\frac{\partial^{2}}{\partial z_{m}^{l} \partial z_{m^{\prime}}^{l^{\prime}}}-\frac{\partial^{2}}{\partial z_{m^{\prime}}^{l} \partial z_{m}^{l^{\prime}}}\right) \partial_{I} \bar{\partial}_{J} U
$$

for all $0 \leq l, l^{\prime} \leq q-1$, all $0 \leq m, m^{\prime} \leq N$ and all $|I|=|J|=q$. Note that since $\Theta$ is assumed to be real, we also have the conjugate equations

$$
0=\left(\frac{\partial^{2}}{\partial \bar{z}_{m}^{l} \partial \bar{z}_{m^{\prime}}^{l^{\prime}}}-\frac{\partial^{2}}{\partial \bar{z}_{m^{\prime}}^{l} \partial \bar{z}_{m}^{l^{\prime}}}\right) \partial_{I} \bar{\partial}_{J} U
$$

for all $0 \leq l, l^{\prime} \leq q-1$, all $0 \leq m, m^{\prime} \leq N$ and all $|I|=|J|=q$.

\subsection{Closed current of bidegree $(p+1, p+1)$ on $P\left(V^{*}\right)$ dual of a closed current of bidegree $(q, q)$ on $P(V)$}

First for $Z$ an algebraic cycle in $P(V)=\mathbb{P}_{N}$ of dimension $p=N-q$, we prove the existence of a closed dual $(p+1, p+1)$-current $[Z]^{*}$ on $P\left(V^{*}\right)$ by using the dual variety $Y=Z^{*} \subset P\left(V^{*}\right)$ of $Z$. Let $\Sigma=\mathcal{C}(Z)$ be the divisor in $G(q, V)$, which is the Chow divisor of $Z$. When $Z$ is irreducible,

$$
\Sigma=\{s \in G(q, V), P(s) \cap Z \neq \varnothing\} .
$$

We have an isomorphism $G(q, V) \simeq G\left(p+1, V^{*}\right)$ which associates to a vector subspace $s \subset V$ its orthogonal $s^{\perp} \subset V^{*}$. We now express the above condition with this dual point of view.

Since $Y=Z^{*} \subset P\left(V^{*}\right)$, we have $Z=Y^{*} \subset P(V)$ (see [10]). Let $I_{Y} \subset P\left(V^{*}\right) \times P(V)$ be the incidence variety associated to $Y$, defined as the Zariski closure of

$$
\left\{([\xi],[x]) \in Y_{\text {reg }} \times P(V),\left(\mathrm{d} \mu_{\xi}\right)^{-1}\left(T_{[\xi]} Y\right) \subset x^{\perp}\right\},
$$

by denoting by $\mu: V^{*} \backslash\{0\} \rightarrow P\left(V^{*}\right)$ the canonical map. The dimension of $I_{Y}$ is $N-1$ and its projection in $P(V)$ is equal to $Z$. Thus for $[x] \in Z$ generic, the Zariski closure of the fiber $\left\{[\xi] \in Y_{\text {reg }},\left(\mathrm{d} \mu_{\xi}\right)^{-1}\left(T_{[\xi]} Y\right) \subset x^{\perp}\right\} \subset$ $P\left(x^{\perp}\right)$ has dimension $N-1-\operatorname{dim} Z=q-1$ and so intersects $P\left(s^{\perp}\right)$ if $x \in s$. If $[\xi]$ is a point of intersection, then we have $\operatorname{dim}\left(\left(\mathrm{d} \mu_{\xi}\right)^{-1}\left(T_{[\xi]} Y\right)+s^{\perp}\right) \leq N$.

We write now $s=\left\{0=\xi_{0}=\ldots=\xi_{p}\right\}$ with $\xi_{0}, \ldots, \xi_{p} \in V^{*}$ and $H=P\left(x^{\perp}\right)$. Thus

$$
\begin{aligned}
& P(s) \cap Z \neq \varnothing \\
\Leftrightarrow & \text { there is } H \supset P\left(\operatorname{vect}\left(\xi_{0}, \ldots, \xi_{p}\right)\right) \text { s. t. } H \supset \mathbb{T}_{[\xi]} Y \text { for some }[\xi] \in Y \cap P\left(\operatorname{vect}\left(\xi_{0}, \ldots, \xi_{p}\right)\right) \\
\Leftrightarrow & \text { there is }[\xi] \in Y \cap P\left(\operatorname{vect}\left(\xi_{0}, \ldots, \xi_{p}\right)\right) \text { s. t. } \operatorname{dim}\left(\mathbb{T}_{[\xi]} Y \cap P\left(\operatorname{vect}\left(\xi_{0}, \ldots, \xi_{p}\right)\right)\right) \geq p+1-\operatorname{codim} Y
\end{aligned}
$$

by setting $\mathbb{T}_{[\xi]} Y=P\left(\left(\mathrm{~d} \mu_{\xi}\right)^{-1}\left(T_{[\xi]} Y\right)\right) \subset P\left(V^{*}\right)$.

Take $W^{\prime} \in G\left(p+2, V^{*}\right)$ such that $P\left(W^{\prime}\right)$ cuts $Y$ transversally in an algebraic variety of dimension $p+1-$ codim $Y$. Then $\Sigma \cap G\left(p+1, W^{\prime}\right)$ is the dual variety in $P\left(W^{\prime *}\right)=G\left(p+1, W^{\prime}\right)$ of $Y \cap P\left(W^{\prime}\right)$, in other words

$$
\Sigma_{\mid P\left(W^{* *}\right)}=\Sigma_{\mid G\left(p+1, W^{\prime}\right)}=\left(Y \cap P\left(W^{\prime}\right)\right)^{*} .
$$

In effect, for $\operatorname{vect}\left(\xi_{0}, \ldots, \xi_{p}\right) \subset W^{\prime}$, if there is $[\xi] \in Y \cap P\left(\operatorname{vect}\left(\xi_{0}, \ldots, \xi_{p}\right)\right)$ satisfying

$$
\mathbb{T}_{[\xi]} Y \cap P\left(W^{\prime}\right) \subset P\left(\operatorname{vect}\left(\xi_{0}, \ldots, \xi_{p}\right)\right),
$$

then

$$
\mathbb{T}_{[\xi]} Y \cap P\left(\operatorname{vect}\left(\xi_{0}, \ldots, \xi_{p}\right)\right)=\mathbb{T}_{[\xi]} Y \cap P\left(W^{\prime}\right)
$$

has dimension $\geq p+1-\operatorname{codim} Y$.

Now denote by $\mathcal{C}^{\prime}$ the Chow transformation of currents of bidegree $(p+1, p+1)$ on $P\left(V^{*}\right)$ and by $\mathcal{C}_{W^{\prime}}^{\prime}$ the Chow transformation of currents of bidegree $(p+1, p+1)$ on $P\left(W^{\prime}\right)$. 
As a closed current of bidegree $(1,1)$ on $P\left(W^{\prime *}\right)$, the current $[\Sigma]_{\mid P\left(W^{\prime *}\right)}$ can be written

$$
[\Sigma]_{\mid P\left(W^{\prime *}\right)}=\mathcal{C}_{W^{\prime}}^{\prime}\left(S_{W^{\prime}}\right)
$$

for some current $S_{W^{\prime}}$ of bidegree $(p+1, p+1)$ on $P\left(W^{\prime}\right)$ (see [25]). These currents satisfy the following property.

Proposition 3. (i) There is a closed $(p+1, p+1)$-current $[Z]^{*}$ on $P\left(V^{*}\right)$ satisfying $[Z]_{\mid P\left(W^{\prime}\right)}^{*}=S_{W^{\prime}}$ for every $W^{\prime} \in G\left(p+2, V^{*}\right)$. Thus $\mathcal{C}([Z])=\mathcal{C}^{\prime}\left([Z]^{*}\right)$.

(ii) For every closed $(q, q)$-current $T$ on $P(V)$, there is a closed $(p+1, p+1)$-current $T^{*}$ on $P\left(V^{*}\right)$ satisfying $\mathcal{C}(T)=\mathcal{C}^{\prime}\left(T^{*}\right)$ on $G(q, V)=G\left(p+1, V^{*}\right)$.

Proof. Every closed $(q, q)$-current $T$ on $P(V)$ can be written $T=\lim _{\nu} Z_{\nu}$ with $Z_{\nu}$ algebraic cycle with complex coefficients on $P(V)$. We set $T^{*}=\lim _{\nu}\left[Z_{\nu}\right]^{*}$ on $P\left(V^{*}\right)$.

Let us express the condition (5) for $\mathcal{C}^{\prime}$, by noting that

$$
\left\{s \in G(q, V), s^{\perp} \subset W^{\prime}\right\}=P\left(W^{\prime *}\right)=\left\{s \in G(q, V), s \supset W^{\prime \perp}\right\}=G\left(1, V / W^{\prime \perp}\right) .
$$

With $p_{W^{\prime}}: P(V) \rightarrow P\left(V / W^{\prime \perp}\right)$ the canonical projection, if $\Theta=\mathcal{C}(T)$ where $T$ is some $(q, q)$-current on $P(V)$, we have

$$
\Theta_{\mid P\left(W^{\prime *}\right)}=\mathcal{C}(T)_{\mid G\left(1, V / W^{\prime \perp}\right)}=p_{W^{\prime} *} T
$$

since $p_{W^{\prime} *} T$ is of bidegree $(1,1)$ on $P\left(V / W^{\prime \perp}\right)$ and the Chow transformation is the identity, when the bidegree is $(1,1)$. Therefore, in that case, the condition (5) reduces to $p_{W^{\prime} *} T$ closed, for every $W^{\prime} \in G\left(p+2, V^{*}\right)$.

Lastly we can extend the method by slicing of subsection 2.2 to the case when $\Theta$ is not necessarily closed. In effect, for $Q$ a function on $\wedge^{q} V=\wedge^{q} \mathbb{C}^{N+1}$, the condition

$$
Q\left(\lambda v_{1} \wedge \ldots \wedge v_{q}\right)=|\lambda|^{2} Q\left(v_{1} \wedge \ldots \wedge v_{q}\right)
$$

for every $\lambda \in \mathbb{C}$ and every $v_{1}, \ldots, v_{q} \in V$ amounts to

$$
\tilde{Q}\left(\lambda v_{n} \wedge v_{n^{\prime}}\right)=|\lambda|^{2} \tilde{Q}\left(v_{n} \wedge v_{n^{\prime}}\right)
$$

for all $1 \leq n, n^{\prime} \leq q$ with $\tilde{Q}\left(v_{n} \wedge v_{n^{\prime}}\right)=Q\left(v_{1} \wedge \ldots \wedge v_{q}\right)$ where the $v_{m}$ for $m \notin\left\{n, n^{\prime}\right\}$ are fixed. Therefore the elements in $\operatorname{im} \mathcal{C}$ must satisfy the following property.

Proposition 4. If a real smooth differential $(1,1)$-form $\Theta$ on $G(q, V)$ is the Chow transform of a real smooth differential $(q, q)$-form $T$ on $P(V)$, then

(i) the condition (5) is satisfied i.e. $\mathrm{d} \Theta_{\mid G(q, W)}=\mathrm{d} \Theta_{\mid P\left(W^{*}\right)}=0$ for all $W \in G(q+1, V)$,

(ii) the linear partial differential equation of order 6 in $\left(v_{1}, \ldots, v_{q}\right)$

$$
0=\frac{\partial}{\partial w_{m, m^{\prime}}^{n, n^{\prime}}} \frac{\partial^{2}}{\partial w_{i, i^{\prime}}^{n, n^{\prime}} \partial \bar{w}_{j, j^{\prime}}^{n, n^{\prime}}}\left\{\mathcal{C}_{W}^{-1}\left(\Theta_{\mid P\left(W^{*}\right)}\right)\left([x] ; \mathrm{d} \pi_{x}\left(v_{1}\right), \ldots, \mathrm{d} \pi_{x}\left(v_{q}\right), \overline{\mathrm{d} \pi_{x}\left(v_{1}\right)}, \ldots, \overline{\mathrm{d} \pi_{x}\left(v_{q}\right)}\right)\right\}
$$

is satisfied with $W=\operatorname{vect}\left(x, v_{1}, \ldots, v_{q}\right)$ for all $x, v_{1}, \ldots, v_{q} \in V$ and all $1 \leq n, n^{\prime} \leq q$ and all $0 \leq$ $m, m^{\prime}, i, i^{\prime}, j, j^{\prime} \leq N$.

\section{The dual of the Chow transformation}

Let $\Omega$ be the fundamental $(1,1)$-form of the Hermitian metric induced in $G(q, V)$, which satisfies $\Omega=$ $\mathrm{dd}^{\mathrm{C}} \log \left\|z^{1} \wedge \ldots \wedge z^{q}\right\|$ for $s=\operatorname{vect}\left(z^{1}, \ldots, z^{q}\right)$.

For $[x] \in P(V)$, the fiber $\varphi^{-1}([x])$ is identified with $G(q-1, V / \mathbb{C} x)$ and thus its dimension is $d=(q-$ 1) $(N+1-q)$. 
For $\Theta$ a $(1,1)$-current on $G(q, V)$, we set

$$
\mathcal{C}^{*}(\Theta)=\varphi_{*} \psi^{*}\left(\Omega^{d+q-1-j} \wedge \Theta\right)
$$

where $j=\max (0,2 q-N-1)$. In effect, we take $j \geq 0$ and we should have $d \leq d+q-j \leq \operatorname{dim} G(q, V)$ i.e. $2 q-N-1 \leq j \leq q$. Then $j \leq d+q-1$.

$\mathcal{C}^{*}(\Theta)$ is a current of bidegree $(q-j, q-j)$ on $P(V)$ and $\mathcal{C}^{*}$ will be a dual of the Chow transformation $\mathcal{C}$. Note that if $j=2 q-N-1$, then $\left(\begin{array}{c}N+1 \\ q-j\end{array}\right)=\left(\begin{array}{c}N+1 \\ q\end{array}\right)$ in such a way that $\operatorname{dim} \wedge^{q-j} V=\operatorname{dim} \wedge^{q} V$.

\subsection{Expression of $\mathcal{C}^{*} \mathcal{C}$ in coordinates}

When $u$ is a smooth differential $(q, q)$-form on $P(V)$, we now calculate

$$
\mathcal{C}^{*} \mathcal{C}(u)=\varphi_{*}\left(\psi^{*} \Omega^{d+q-1-j} \wedge \psi^{*} \psi_{*} \varphi^{*} u\right) .
$$

We use that the direct image by a submersion of a smooth differential form can be calculated by integration along the fibers. With $v_{1}, \ldots, v_{q-j} \in V$ and $\pi: V-\{0\} \rightarrow P(V)$ the canonical map, we obtain

$$
\pi^{*}\left(\mathcal{C}^{*} \mathcal{C}(u)\right)_{x}\left(v_{1}, \ldots, v_{q-j}, \bar{v}_{1}, \ldots, \bar{v}_{q-j}\right)=\int_{s \in \varphi^{-1}([x])}\left(\Omega^{d+q-1-j} \wedge \mathcal{C}(u)\right)_{s}\left(\sigma_{1}, \ldots, \sigma_{q-j}, \bar{\sigma}_{1}, \ldots, \bar{\sigma}_{q-j}, \ldots\right)
$$

where $\sigma_{k} \in T_{s} G(q, V)$ is such that $\left(\mathrm{d} \pi_{x}\left(v_{k}\right), \sigma_{k}\right) \in T_{([x], s)} \Gamma$. We now express the function $A(s)$ for $s \ni x$ such that

$$
\left(\Omega_{s}^{d+q-1-j} \wedge \mathcal{C}(u)_{s}\right)\left(\sigma_{1}, \ldots, \sigma_{q-j}, \bar{\sigma}_{1}, \ldots, \bar{\sigma}_{q-j}, \ldots\right)_{\mid T_{s} \varphi^{-1}([x])}=A(s) \Omega_{s \mid T_{s} \varphi^{-1}([x])}^{d} .
$$

Assume that $\left(e_{0}, \ldots, e_{N}\right)$ is an orthonormal basis of $V$ and that $[x]=\left[e_{0}\right]$ and $s=\operatorname{vect}\left(e_{l}\right)_{l<q}$. Coordinates of $G(q, V)$ are the $z_{l}^{k}$ for $k<q \leq l$, with the associated point of $G(q, V)$ equal to

$$
\operatorname{vect}\left(e_{k}+\sum_{q \leq l} z_{l}^{k} e_{l}\right)_{k<q}
$$

Then $\varphi^{-1}\left(\left[e_{0}\right]\right)$ is defined by $z_{l}^{0}=0$ for $q \leq l$. At this $s$, we can take

$$
\begin{aligned}
\sigma_{k} & =\frac{\mathrm{d}}{\mathrm{d} t} \operatorname{vect}\left(e_{0}+t v_{k}, e_{1}, \ldots, e_{q-1}\right)_{\mid t=0} \\
& =\frac{\mathrm{d}}{\mathrm{d} t} \operatorname{vect}\left(e_{0}+t v_{k, 0} e_{0}+t \sum_{q \leq l} v_{k, l} e_{l}, e_{1}, \ldots, e_{q-1}\right)_{\mid t=0} \\
& =\sum_{q \leq l} \frac{\mathrm{d}}{\mathrm{d} t}\left(\frac{t v_{k, l}}{1+t v_{k, 0}}\right)_{\mid t=0} \frac{\partial}{\partial z_{l}^{0}}=\sum_{q \leq l} v_{k, l} \frac{\partial}{\partial z_{l}^{0}} .
\end{aligned}
$$

At this $s$, we have $\Omega_{s}=\frac{i}{2 \pi} \sum_{k<q \leq l} \mathrm{~d} z_{l}^{k} \wedge \mathrm{d} \bar{z}_{l}^{k}$ and

$$
\Omega_{s \mid T_{s} \varphi^{-1}\left(\left[e_{0}\right]\right)}=\frac{i}{2 \pi} \sum_{1 \leq k<q \leq l} \mathrm{~d} z_{l}^{k} \wedge \mathrm{d} \bar{z}_{l}^{k} .
$$

So there exists a constant $K=(-1)^{(q-j)(q-j-1) / 2}\left(\frac{i}{2 \pi}\right)^{d} d !$ satisfying

$$
K A(s)=\left(\Omega_{s}^{d+q-1-j} \wedge \mathcal{C}(u)_{s}\right)\left(\sigma_{1}, \bar{\sigma}_{1}, \ldots, \sigma_{q-j}, \bar{\sigma}_{q-j}, \ldots, \frac{\partial}{\partial z_{l}^{k}}, \frac{\partial}{\partial \bar{z}_{l}^{k}}, \ldots\right)
$$

where $1 \leq k<q$ and $q \leq l$. Now recall (see [25]) the expression of $\mathcal{C}(u)$ with the homogeneous coordinates.

The inverse image of $u$ by the canonical map $\pi: V \rightarrow P(V)$ is written as

$$
\pi^{*} u=\sum_{|I|=|J|=q} u_{I, J} \mathrm{~d} x_{I} \wedge \mathrm{d} \bar{x}_{J}
$$


with functions $u_{I, J}$ satisfying in particular the property of homogeneity $u_{I, J}(\lambda x)=|\lambda|^{-2 q} u_{I, J}(x)$ for $\lambda \in \mathbb{C}^{*}$.

Denote by $\tau: V^{q} \rightarrow G(q, V)$ the map which associates to $z=\left(z^{0}, \ldots, z^{q-1}\right)$ in the product the vector subspace $\tau(z)=\operatorname{vect}\left(z^{0}, \ldots, z^{q-1}\right)$. The inverse image by $\tau$ of the Chow transform $\mathcal{C}(u)$ is then written as

$$
\tau^{*} \mathcal{C}(u)=\sum_{\substack{0 \leq k, m \leq q-1 \\ 0 \leq n, l \leq N}} \mathcal{C}_{n l}^{k m}(u) \mathrm{d} z_{n}^{k} \wedge \mathrm{d} \bar{z}_{l}^{m} .
$$

The coefficients $\mathcal{C}_{n l}^{\mathrm{km}}(u)$ can be expressed by means of the $u_{I, J}$ in the following way :

$$
\mathcal{C}_{n l}^{k m}(u)(z)=(-1)^{k+m} \sum_{|I|=|J|=q-1} \tilde{u}_{n I, l J}(z) z_{I}^{\hat{k}} \bar{z}_{J}^{\hat{m}}
$$

with $z_{I}^{\hat{k}}=\operatorname{det}\left(z_{i_{\alpha}}^{k^{\prime}}\right){\substack{1 \leq \alpha \leq q-1 \\ 0 \leq k^{\prime} \leq q-1, k^{\prime} \neq k}}_{\substack{1 \\ \text { and }}}$ and with the transform

$$
\tilde{u}_{n I, l J}(z)=\int_{t \in \mathbb{S}^{2 q-1}} u_{n I, l J}(t \cdot z) \Phi(t)
$$

where $t \cdot z=t_{1} z^{0}+\ldots+t_{q} z^{q-1}$ and

$$
\begin{aligned}
\Phi(t)=\frac{i}{4 \pi} \sum_{1 \leq k \leq q}(-1)^{k-1}\left(t_{k} \mathrm{~d} t_{1} \wedge \ldots \wedge \widehat{\mathrm{d} t_{k}} \wedge \ldots \wedge\right. & \mathrm{d} t_{q} \wedge \mathrm{d} \bar{t}_{1} \wedge \ldots \wedge \mathrm{d} \bar{t}_{q} \\
& \left.+(-1)^{q} \bar{t}_{k} \mathrm{~d} t_{1} \wedge \ldots \wedge \mathrm{d} t_{q} \wedge \mathrm{d} \bar{t}_{1} \wedge \ldots \wedge \widehat{\mathrm{d}}_{k} \wedge \ldots \wedge \mathrm{d} \bar{t}_{q}\right) .
\end{aligned}
$$

Set $f_{n I, l J}([x])=\|x\|^{2 q} u_{n I, l J}(x)$ and let $\mathcal{R}_{q-1}\left(f_{n I, l J}\right)$ be the Radon transform of $f_{n I, l J}$ obtained by integration of that function in the projective subspaces of $P(V)$ of dimension $q-1$. Also

$$
\mathcal{R}_{q-1}\left(f_{n I, l J}\right)_{\tau(z)}=\alpha_{q} \tilde{u}_{n I, l J}(z) \Delta(z)
$$

with $\alpha_{q}=\frac{(q-1) ! i^{q^{2}-1}}{(2 \pi) q^{-1}}$ and $\Delta(z)$ the Gram determinant of $z$.

But since we use the coordinates $z_{l}^{k}$ for $k<q \leq l$, we can write

$$
\mathcal{C}(u)=\sum_{\substack{0 \leq k, m \leq q-1 \\ q \leq n, l \leq N}} \mathcal{C}_{n l}^{k m}(u) \mathrm{d} z_{n}^{k} \wedge \mathrm{d} \bar{z}_{l}^{m} .
$$

Moreover at $s=\operatorname{vect}\left(e_{0}, e_{1}, \ldots, e_{q-1}\right)$ we have $z_{I}^{\hat{k}}=0$ except for $I=\{0, \ldots, k-1, k+1, \ldots, q-1\}$ and in the same way $\bar{z}_{J}^{\hat{m}}=0$ except for $J=\{0, \ldots, m-1, m+1, \ldots, q-1\}$. Thus at that $z$ we have

$$
\mathcal{C}_{n l}^{k m}(u)(z)=(-1)^{k+m} \tilde{u}_{n \hat{k}, l \hat{m}}(z)=\tilde{u}_{\{0, \ldots, k-1, n, k+1, \ldots, q-1\},\{0, \ldots, m-1, l, m+1, \ldots, q-1\}}(z)
$$

and therefore

$$
\begin{aligned}
& \Omega_{s}^{d+q-1-j} \wedge \mathcal{C}(u)_{s} \\
= & \left(\frac{i}{2 \pi} \sum_{\substack{0 \leq k \leq q-1 \\
q \leq l \leq N}} \mathrm{~d} z_{l}^{k} \wedge \mathrm{d} \bar{z}_{l}^{k}\right)^{d+q-1-j} \wedge \sum_{\substack{0 \leq k, m \leq q-1 \\
q \leq n, l \leq N}}(-1)^{k+m} \tilde{u}_{n \hat{k}, l \hat{m}}(z) \mathrm{d} z_{n}^{k} \wedge \mathrm{d} \bar{z}_{l}^{m} \\
= & \left(\frac{i}{2 \pi}\right)^{d+q-1-j} \sum \mathrm{d} z_{l_{1}}^{k_{1}} \wedge \mathrm{d} \bar{z}_{l_{1}}^{k_{1}} \wedge \ldots \wedge \mathrm{d} z_{l_{d+q-1-j}}^{k_{d+q-1-j}} \wedge \mathrm{d} \bar{z}_{l_{d+q-1-j}}^{k_{d+q-1-j}} \wedge(-1)^{k+m} \tilde{u}_{n \hat{k}, l \hat{m}}(z) \mathrm{d} z_{n}^{k} \wedge \mathrm{d} \bar{z}_{l}^{m}
\end{aligned}
$$

where $0 \leq k_{1}, \ldots, k_{d+q-1-j}, k, m \leq q-1$ and $q \leq l_{1}, \ldots, l_{d+q-1-j}, n, l \leq N$.

On the other hand, with $1 \leq k \leq q-1$ and $q \leq l \leq N$, we have

$$
\begin{gathered}
\sigma_{1} \wedge \bar{\sigma}_{1} \wedge \ldots \wedge \sigma_{q-j} \wedge \bar{\sigma}_{q-j} \wedge \ldots \wedge \frac{\partial}{\partial z_{l}^{k}} \wedge \frac{\partial}{\partial \bar{z}_{l}^{k}} \wedge \ldots \\
=\left(\sum_{q \leq i_{1}} v_{1, i_{1}} \frac{\partial}{\partial z_{i_{1}}^{0}}\right) \wedge\left(\sum_{q \leq n_{1}} \bar{v}_{1, n_{1}} \frac{\partial}{\partial \bar{z}_{n_{1}}^{0}}\right) \wedge \ldots \wedge\left(\sum_{q \leq i_{1}} v_{q-j, i_{1}} \frac{\partial}{\partial z_{i_{1}}^{0}}\right) \wedge\left(\sum_{q \leq n_{1}} \bar{v}_{q-j, n_{1}} \frac{\partial}{\partial \bar{z}_{n_{1}}^{0}}\right) \wedge \ldots
\end{gathered}
$$




$$
\begin{array}{r}
\wedge \frac{\partial}{\partial z_{l}^{k}} \wedge \frac{\partial}{\partial \bar{z}_{l}^{k}} \wedge \ldots \\
=\sum v_{1, i_{1}} \bar{v}_{1, n_{1}} \ldots v_{q-j, i_{q-j}} \bar{v}_{q-j, n_{q-j}} \frac{\partial}{\partial z_{i_{1}}^{0}} \wedge \frac{\partial}{\partial \bar{z}_{n_{1}}^{0}} \wedge \ldots \wedge \frac{\partial}{\partial z_{i_{q-j}}^{0}} \wedge \frac{\partial}{\partial \bar{z}_{n_{q-j}}^{0}} \wedge \frac{\partial}{\partial z_{l_{1}^{\prime}}^{k_{1}^{\prime}}} \wedge \frac{\partial}{\partial \bar{z}_{l_{1}^{\prime}}^{k_{1}^{\prime}}} \wedge \ldots \\
\wedge \frac{\partial}{\partial z_{l_{d}^{\prime}}^{k_{d}^{\prime}}} \wedge \frac{\partial}{\partial \bar{z}_{l_{d}^{\prime}}^{k_{d}^{\prime}}}
\end{array}
$$

where $q \leq i_{1}, \ldots, i_{q-j} \leq N$ and $q \leq n_{1}, \ldots, n_{q-j} \leq N$ and where the vectors $\frac{\partial}{\partial z_{l_{1}^{\prime}}^{k_{1}^{\prime}}}, \ldots, \frac{\partial}{\partial z_{l_{d}^{\prime}}^{k^{\prime}}}$ with $1 \leq k_{1}^{\prime}, \ldots, k_{d}^{\prime} \leq$ $q-1$ and $q \leq l_{1}^{\prime}, \ldots, l_{d}^{\prime} \leq N$ constitute a basis of $T_{s} \varphi^{-1}\left(\left[e_{0}\right]\right)$

As a consequence, when we calculate $A(s)$ by means of the formula (13), we must choose the indices such that the following equalities between sets are satisfied

$$
\left\{\frac{\partial}{\partial z_{i_{1}}^{0}}, \ldots, \frac{\partial}{\partial z_{i_{q-j}}^{0}}, \frac{\partial}{\partial z_{l_{1}^{\prime}}^{k_{1}^{\prime}}}, \ldots, \frac{\partial}{\partial z_{l_{d}^{\prime}}^{k_{d}^{\prime}}}\right\}=\left\{\frac{\partial}{\partial z_{l_{1}}^{k_{1}}}, \ldots, \frac{\partial}{\partial z_{l_{d+q-1-j}}^{k_{d+q-1-j}}}, \frac{\partial}{\partial z_{n}^{k}}\right\}
$$

and

$$
\left\{\frac{\partial}{\partial \bar{z}_{n_{1}}^{0}}, \ldots, \frac{\partial}{\partial \bar{z}_{n_{q-j}}^{0}}, \frac{\partial}{\partial \bar{z}_{l_{1}^{\prime}}^{k_{1}^{\prime}}}, \ldots, \frac{\partial}{\partial \bar{z}_{l_{d}^{\prime}}^{k_{d}^{\prime}}}\right\}=\left\{\frac{\partial}{\partial \bar{z}_{l_{1}}^{k_{1}}}, \ldots, \frac{\partial}{\partial \bar{z}_{l_{d+q-1-j}}^{k_{d+1-1-j}}}, \frac{\partial}{\partial \bar{z}_{l}^{m}}\right\}
$$

The indices in the lists $z_{i_{1}}^{0}, \ldots, z_{i_{q-j}}^{0}, z_{l_{1}^{\prime}}^{k_{1}^{\prime}}, \ldots, z_{l_{d}^{\prime}}^{k_{d}^{\prime}}$ and $\bar{z}_{n_{1}}^{0}, \ldots, \bar{z}_{n_{q-j}}^{0}, \bar{z}_{l_{1}^{\prime}}^{k_{1}^{\prime}}, \ldots, \bar{z}_{l_{d}^{\prime}}^{k_{d}^{\prime}}$ should differ by at most one element. A first possibility is

$$
z_{i_{1}}^{0}, \ldots, z_{i_{\alpha-1}}^{0}, z_{i_{\alpha+1}}^{0}, \ldots, z_{i_{q-j}}^{0}, z_{l_{1}^{\prime}}^{k_{1}^{\prime}}, \ldots, z_{l_{d}^{\prime}}^{k_{d}^{\prime}} \text { and } \bar{z}_{i_{1}}^{0}, \ldots, \bar{z}_{i_{\alpha-1}}^{0}, \bar{z}_{i_{\alpha+1}}^{0}, \ldots, \bar{z}_{i_{q-j}}^{0}, \bar{z}_{l_{1}^{\prime}}^{k_{1}^{\prime}}, \ldots, \bar{z}_{l_{d}^{\prime}}^{k_{d}^{\prime}} .
$$

We obtain a first term $K_{1} A_{1}(s)$ with $K_{1}=\left(\frac{i}{2 \pi}\right)^{d+q-1-j}(d+q-1-j)$ ! and with

$$
\begin{array}{r}
A_{1}(s)=\sum_{\alpha, \beta} \sum_{I} \sum_{\sigma, \tau} \sum_{i_{\alpha}, n_{\beta}} \varepsilon(\sigma) \varepsilon(\tau)(-1)^{\alpha+\beta} v_{1, i_{\sigma(1)}} \ldots v_{\alpha-1, i_{\sigma(\alpha-1)}} v_{\alpha, i_{\alpha}} v_{\alpha+1, i_{\sigma(\alpha+1)}} \ldots v_{q-j, i_{\sigma(q-j)}} \\
\cdot \bar{v}_{1, i_{\tau(1)}^{\prime}} \ldots \bar{v}_{\beta-1, i_{\tau(\beta-1)}^{\prime}} \bar{v}_{\beta, n_{\beta}} \bar{v}_{\beta+1, i_{\tau(\beta+1)}^{\prime}} \ldots \bar{v}_{q-j, i_{\tau(q-j)}^{\prime}}(-1)^{0+0} \tilde{u}_{i_{\alpha} \hat{0}, n_{\beta} \hat{0}}(z)
\end{array}
$$

where $1 \leq \alpha, \beta \leq q-j$ and $I=\left(i_{1}, \ldots, i_{\alpha-1}, i_{\alpha+1}, \ldots, i_{q-j}\right)=\left(i_{1}^{\prime}, \ldots, i_{\beta-1}^{\prime}, i_{\beta+1}^{\prime}, \ldots, i_{q-j}^{\prime}\right)$ is a strictly increasing sequence of $q-j-1$ integers between $q$ and $N$ and where $\sigma, \tau$ are permutations of $\{1, \ldots, q-j\}$ such that $\sigma(\alpha)=\alpha$ et $\tau(\beta)=\beta$.

The second possibility is for some $\delta \in\{1, \ldots, d\}$

$$
z_{i_{1}}^{0}, \ldots, z_{i_{q-j}}^{0}, z_{l_{1}^{\prime}}^{k_{1}^{\prime}}, \ldots, z_{l_{\delta-1}^{\prime}}^{k_{\delta-1}^{\prime}}, z_{l_{\delta+1}^{\prime}}^{k_{\delta+1}^{\prime}}, \ldots, z_{l_{d}^{\prime}}^{k_{d}^{\prime}} \text { and } \bar{z}_{i_{1}}^{0}, \ldots, \bar{z}_{i_{q-j}}^{0}, \bar{z}_{l_{1}^{\prime}}^{k_{1}^{\prime}}, \ldots, \bar{z}_{l_{\delta-1}^{\prime}}^{k_{\delta-1}^{\prime}}, \bar{z}_{l_{\delta+1}^{\prime}}^{k_{\delta+1}^{\prime}}, \ldots, \bar{z}_{l_{d}^{\prime}}^{k_{d}^{\prime}} .
$$

We obtain a second term $K_{2} A_{2}(s)$ with $K_{2}=\left(\frac{i}{2 \pi}\right)^{d+q-1-j}(d+q-1-j) !=K_{1}$ and with

$$
A_{2}(s)=\sum_{I} \sum_{\sigma, \tau} \varepsilon(\sigma) \varepsilon(\tau) v_{1, i_{\sigma(1)}} \ldots v_{q-j, i_{\sigma(q-j)}} \bar{v}_{1, i_{\tau(1)}} \ldots \bar{v}_{q-j, i_{\tau(q-j)}} \sum_{\substack{1 \leq k^{\prime} \leq q-1 \\ q \leq l^{\prime} \leq N}}(-1)^{k^{\prime}+k^{\prime}} \tilde{u}_{l^{\prime} \widehat{k^{\prime}, l^{\prime} \bar{k}^{\prime}}}(z)
$$

where $I=\left(i_{1}, \ldots, i_{q-j}\right)$ with $q \leq i_{1}<\ldots<i_{q-j} \leq N$ and $\sigma, \tau$ are permutations of $\{1, \ldots, q-j\}$.

Denote by $p_{s^{\perp}}: \mathbb{C}^{N+1} \rightarrow \mathbb{C}^{N+1}$ the orthogonal projection onto $s^{\perp}=\operatorname{vect}\left(e_{q}, \ldots, e_{N}\right)$, then

$$
p_{S^{\perp}}^{*}\left(\frac{i}{2 \pi} \partial \bar{\partial}\left(\|x\|^{2}\right)\right)^{q-j-1}=C_{1} \sum_{|I|=q-j-1} \mathrm{~d} x_{I} \wedge \mathrm{d} \bar{x}_{I}
$$

where $I=\left(i_{1}, \ldots, i_{q-j-1}\right)$ with $q \leq i_{1}<\ldots<i_{q-j-1} \leq N$ and $C_{1}=\frac{i^{(q-j-1)^{2}}}{(2 \pi)^{q-j-1}}(q-j-1) !$ is a constant. We obtain by formula (14)

$$
C_{1} A_{1}(s)=\sum_{\alpha, \beta} p_{s^{\perp}}^{*}\left(\frac{i}{2 \pi} \partial \bar{\partial}\left(\|x\|^{2}\right)\right)_{e_{0}}^{q-j-1}\left(v_{1}, \ldots, v_{\alpha-1}, v_{\alpha+1}, \ldots, v_{q-j}, \bar{v}_{1}, \ldots, \bar{v}_{\beta-1}, \bar{v}_{\beta+1}, \ldots, \bar{v}_{q-j}\right)
$$




$$
\cdot(-1)^{\alpha+\beta} \int_{t \in \mathbb{S}^{2 q-1}} \sum_{i_{\alpha}, n_{\beta}}\left(\pi^{*} u\right)_{t \cdot z}\left(e_{i_{\alpha}}, e_{1}, \ldots, e_{q-1}, \bar{e}_{n_{\beta}}, \bar{e}_{1}, \ldots, \bar{e}_{q-1}\right) v_{\alpha, i_{\alpha}} \bar{v}_{\beta, n_{\beta}} \Phi(t) .
$$

In other words $C_{1} A_{1}(s)$ is the value at $\left(v_{1}, \ldots, v_{q-j}, \bar{v}_{1}, \ldots, \bar{v}_{q-j}\right)$ of

$$
(-1)^{q-j-1} p_{s^{\perp}}^{*}\left(\frac{i}{2 \pi} \partial \bar{\partial}\left(\|x\|^{2}\right)\right)_{x}^{q-j-1} \wedge \int_{t \in \mathbb{S} 2 q-1}\left(\pi^{*} u\right)_{t \cdot z}\left(., z^{1}, \ldots, z^{q-1}, ., \bar{z}^{1}, \ldots, \bar{z}^{q-1}\right) \Phi(t) .
$$

Note that

$$
\left(\pi^{*} u\right)_{t \cdot z}\left(e_{0}, e_{1}, \ldots, e_{q-1}, \bar{e}_{n_{\beta}}, \bar{e}_{1}, \ldots, \bar{e}_{q-1}\right)=0=\left(\pi^{*} u\right)_{t \cdot z}\left(e_{i_{\alpha}}, e_{1}, \ldots, e_{q-1}, \bar{e}_{0}, \bar{e}_{1}, \ldots, \bar{e}_{q-1}\right)
$$

since $\mathrm{d} \pi_{t \cdot z}\left(e_{0}\right), \ldots, \mathrm{d} \pi_{t \cdot z}\left(e_{q-1}\right)$ are linearly dependent, thanks to $t_{1} \mathrm{~d} \pi_{t \cdot z}\left(e_{0}\right)+\ldots+t_{q} \mathrm{~d} \pi_{t \cdot z}\left(e_{q-1}\right)=$ $\mathrm{d} \pi_{t \cdot z}\left(t_{1} e_{0}+\ldots+t_{q} e_{q-1}\right)=\mathrm{d} \pi_{t \cdot z}(t \cdot z)=0$.

On the other hand, for the second term, we write

$$
p_{s^{\perp}}^{*}\left(\frac{i}{2 \pi} \partial \bar{\partial}\left(\|x\|^{2}\right)\right)^{q-j}=C_{2} \sum_{|I|=q-j} \mathrm{~d} x_{I} \wedge \mathrm{d} \bar{x}_{I}
$$

where $I=\left(i_{1}, \ldots, i_{q-j}\right)$ with $q \leq i_{1}<\ldots<i_{q-j} \leq N$ and $C_{2}=\frac{i^{(q-j)^{2}}}{(2 \pi)^{q-j}}(q-j) !$ is a constant. We obtain by (14)

$$
\begin{array}{r}
C_{2} A_{2}(s)=p_{s^{\perp}}^{*}\left(\frac{i}{2 \pi} \partial \bar{\partial}\left(\|x\|^{2}\right)\right)_{e_{0}}^{q-j}\left(v_{1}, \ldots, v_{q-j}, \bar{v}_{1}, \ldots, \bar{v}_{q-j}\right) \int_{\substack { t \in \mathbb{S}^{2} q-1 \\
\begin{subarray}{c}{1 \leq k^{\prime} \leq q-1 \\
0 \leq l^{\prime} \leq N{ t \in \mathbb { S } ^ { 2 } q - 1 \\
\begin{subarray} { c } { 1 \leq k ^ { \prime } \leq q - 1 \\
0 \leq l ^ { \prime } \leq N } }\end{subarray}}\left\{\sum_{k^{\prime}}\right. \\
\left.\left(\pi^{*} u\right)_{t \cdot z}\left(e_{0}, \ldots, e_{k^{\prime}-1}, e_{l^{\prime}}, e_{k^{\prime}+1}, \ldots, e_{q-1}, \bar{e}_{0}, \ldots, \bar{e}_{k^{\prime}-1}, \bar{e}_{l^{\prime}}, \bar{e}_{k^{\prime}+1}, \ldots, \bar{e}_{q-1}\right)\right\} \Phi(t) .
\end{array}
$$

In other words $C_{2} A_{2}(s)$ is the value at $\left(v_{1}, \ldots, v_{q-j}, \bar{v}_{1}, \ldots, \bar{v}_{q-j}\right)$ of

$$
\begin{array}{r}
p_{s^{\perp}}^{*}\left(\frac{i}{2 \pi} \partial \bar{\partial}\left(\|x\|^{2}\right)\right)_{x}^{q-j} \int_{t \in \mathbb{S}^{2 q-1}} \operatorname{tr}\left\{\sum _ { 1 \leq k ^ { \prime } \leq q - 1 } ( \pi ^ { * } u ) _ { t \cdot z } \left(z^{0}, \ldots, z^{k^{\prime}-1}, ., z^{k^{\prime}+1},\right.\right. \\
\left.\left.\ldots, z^{q-1}, \bar{z}^{0}, \ldots, \bar{z}^{k^{\prime}-1}, ., \bar{z}^{k^{\prime}+1}, \ldots, \bar{z}^{q-1}\right)\right\} \Phi(t)
\end{array}
$$

where we denote by tr the trace with respect to $\frac{i}{2} \partial \bar{\partial}\left(\|x\|^{2}\right)$ of the differential $(1,1)$-form in $\{$.$\} .$

Now we write $p_{s^{\perp}}^{*}\left(\frac{i}{2 \pi} \partial \bar{\partial}\left(\|x\|^{2}\right)\right)=\frac{i}{2 \pi} \partial \bar{\partial}\left(\left\|p_{s^{\perp}}(x)\right\|^{2}\right)=\frac{i}{2 \pi} \partial \bar{\partial}\left(d(x, s)^{2}\right)$ with $d(x, s)$ the distance of $x$ to the vector subspace $s$ of $\mathbb{C}^{N+1}$. We have

$$
d(x, s)^{2}=\frac{\Delta\left(x, z^{0}, \ldots, z^{q-1}\right)}{\Delta\left(z^{0}, \ldots, z^{q-1}\right)}=\sum_{0 \leq i, i^{\prime} \leq N} x_{i} \bar{x}_{i^{\prime}} M_{i i^{\prime}}
$$

where $\Delta\left(z^{0}, \ldots, z^{q-1}\right)$ is the Gram determinant of $z^{0}, \ldots, z^{q-1}$ and where $\left(M_{i i^{\prime}}\right)_{i, i^{\prime}}$ is a Hermitian matrix depending only on $s=\operatorname{vect}\left(z^{0}, \ldots, z^{q-1}\right)$. Then

$$
\begin{aligned}
& p_{s^{\perp}}^{*}\left(\frac{i}{2 \pi} \partial \bar{\partial}\left(\|x\|^{2}\right)\right)^{q-j-1} \\
= & \left(\frac{i}{2 \pi}\right)^{q-j-1} \sum M_{i_{1} i_{1}^{\prime}} \ldots M_{i_{q-j-1} i_{q-j-1}^{\prime}} \mathrm{d} x_{i_{1}} \wedge \mathrm{d} \bar{x}_{i_{1}^{\prime}} \wedge \cdots \wedge \mathrm{d} x_{i_{q-j-1}} \wedge \mathrm{d} \bar{x}_{i_{q-j-1}^{\prime}} \\
= & C_{1} \sum_{|I|=\left|I^{\prime}\right|=q-j-1} \operatorname{det}\left(M_{i i^{\prime}}\right)_{i \in I, i^{\prime} \in I^{\prime}} \mathrm{d} x_{I} \wedge \mathrm{d} \bar{x}_{I^{\prime}}
\end{aligned}
$$

while

$$
\begin{aligned}
& \int_{t \in \mathbb{S}^{2 q-1}}\left(\pi^{*} u\right)_{t \cdot z}\left(., z^{1}, \ldots, z^{q-1}, ., \bar{z}^{1}, \ldots, \bar{z}^{q-1}\right) \Phi(t) \\
= & \sum_{n, l}\left(\int_{t \in \mathbb{S}^{2 q-1}}\left\{\sum_{|J|=\left|J^{\prime}\right|=q-1}\left(\pi^{*} u\right)_{t \cdot z}\left(e_{n}, e_{j_{1}}, \ldots, e_{j_{q-1}}, \bar{e}_{l}, \bar{e}_{j_{1}^{\prime}}, \ldots, \bar{e}_{j_{q-1}^{\prime}}\right) z_{J}^{0} \bar{z}_{J^{\prime}}^{\hat{0}}\right\} \Phi(t)\right) \mathrm{d} x_{n} \wedge \mathrm{d} \bar{x}_{l} \\
= & \sum_{n, l}\left((-1)^{0+0} \sum_{|J|=\left|J^{\prime}\right|=q-1} \tilde{u}_{n J, l J^{\prime}}(z) z_{J}^{\hat{0}} \bar{z}_{J^{\prime}}^{\hat{0}}\right) \mathrm{d} x_{n} \wedge \mathrm{d} \bar{x}_{l}
\end{aligned}
$$


$=\sum_{n, l} \mathcal{C}_{n l}^{00}(u)(z) \mathrm{d} x_{n} \wedge \mathrm{d} \bar{x}_{l}$.

With $C_{1}^{\prime}=1$, we conclude that $C_{1}^{\prime} A_{1}(s)$ is the value at $\left(v_{1}, \ldots, v_{q-j}, \bar{v}_{1}, \ldots, \bar{v}_{q-j}\right)$ of

$$
\begin{aligned}
& (-1)^{q-j-1} \sum_{|I|=\left|I^{\prime}\right|=q-j-1} \operatorname{det}\left(M_{i i^{\prime}}\right)_{i \in I, i^{\prime} \in I^{\prime}} \mathrm{d} x_{I} \wedge \mathrm{d} \bar{x}_{I^{\prime}} \wedge \sum_{n, l} \mathcal{C}_{n l}^{00}(u)(z) \mathrm{d} x_{n} \wedge \mathrm{d} \bar{x}_{l} \\
& =\sum_{|I|=\left|I^{\prime}\right|=q-j}\left\{\sum_{1 \leq \alpha, \beta \leq q-j}(-1)^{\alpha+\beta} \operatorname{det}\left(M_{i i^{\prime}}\right)_{\substack{i \in L_{i}, i \neq i \\
i^{\prime} \in I^{\prime}, i^{\prime} i_{\beta}^{\prime}}} \mathcal{C}_{i_{\alpha} i_{\beta}^{\prime}}^{00}(u)(z)\right\} \mathrm{d} x_{I} \wedge \mathrm{d} \bar{x}_{I^{\prime}}
\end{aligned}
$$

where $I=\left(i_{1}, \ldots, i_{q-j}\right)$ with $0 \leq i_{1}<\ldots<i_{q-j} \leq N$ and where $I^{\prime}=\left(i_{1}^{\prime}, \ldots, i_{q-j}^{\prime}\right)$ with $0 \leq i_{1}^{\prime}<\ldots<i_{q-j}^{\prime} \leq N$.

For the second term we have

$$
\begin{aligned}
p_{s^{\perp}}^{*}\left(\frac{i}{2 \pi} \partial \bar{\partial}\left(\|x\|^{2}\right)\right)^{q-j} & =\left(\frac{i}{2 \pi}\right)^{q-j} \sum M_{i_{1} i_{1}^{\prime}} \ldots M_{i_{q-j} i_{q-j}^{\prime}} \mathrm{d} x_{i_{1}} \wedge \mathrm{d} \bar{x}_{i_{1}^{\prime}} \wedge \cdots \wedge \mathrm{d} x_{i_{q-j}} \wedge \mathrm{d} \bar{x}_{i_{q-j}^{\prime}} \\
& =C_{2} \sum_{|I|=\left|I^{\prime}\right|=q-j} \operatorname{det}\left(M_{i i^{\prime}}\right)_{i \in I, i^{\prime} \in I^{\prime}} \mathrm{d} x_{I} \wedge \mathrm{d} \bar{x}_{I^{\prime}}
\end{aligned}
$$

while

$$
\begin{array}{r}
\int_{t \in \mathbb{S}^{2 q-1}} \operatorname{tr}\left\{\sum_{1 \leq k^{\prime} \leq q-1}\left(\pi^{*} u\right)_{t \cdot z}\left(z^{0}, \ldots, z^{k^{\prime}-1}, ., z^{k^{\prime}+1}, \ldots, z^{q-1}, \bar{z}^{0}, \ldots, \bar{z}^{k^{\prime}-1}, ., \bar{z}^{k^{\prime}+1}, \ldots, \bar{z}^{q-1}\right)\right\} \Phi(t) \\
=\int_{t \in \mathbb{S}^{2 q-1}}\left\{\sum _ { 1 \leq k ^ { \prime } \leq q - 1 } \sum _ { 0 \leq l \leq N } ( - 1 ) ^ { k ^ { \prime } + k ^ { \prime } } \sum _ { | J | = | J ^ { \prime } | = q - 1 } ( \pi ^ { * } u ) _ { t \cdot z } \left(e_{j_{0}}, \ldots, e_{j_{k^{\prime}-1}}, e_{l}, e_{j_{k^{\prime}+1}}, \ldots, e_{j_{q-1}}, \bar{e}_{j_{0}^{\prime}}, \ldots,\right.\right. \\
\left.\left.\bar{e}_{j_{k^{\prime}-1}^{\prime}}, \bar{e}_{l}, \bar{e}_{j_{k^{\prime}+1}}, \ldots, \bar{e}_{j_{q-1}^{\prime}}\right) z_{J}^{\hat{k}^{\prime}} \bar{z}_{J^{\prime}}^{\hat{k}^{\prime}}\right\} \Phi(t)
\end{array}
$$

is equal to

$$
\sum_{1 \leq k^{\prime} \leq q-1} \sum_{0 \leq l \leq N}(-1)^{k^{\prime}+k^{\prime}} \sum_{|J|=\left|J^{\prime}\right|=q-1} \tilde{u}_{l J, l J^{\prime}}(z) z_{J}^{\hat{k}_{J}^{\prime}} \bar{z}_{J^{\prime}}^{\hat{k}^{\prime}}=\sum_{1 \leq k^{\prime} \leq q-1} \sum_{0 \leq l \leq N} \mathcal{C}_{l l}^{k^{\prime} k^{\prime}}(u)(z) .
$$

With $C_{2}^{\prime}=1$, we conclude that $C_{2}^{\prime} A_{2}(s)$ is the value at $\left(v_{1}, \ldots, v_{q-j}, \bar{v}_{1}, \ldots, \bar{v}_{q-j}\right)$ of

$$
\left(\sum_{|I|=\left|I^{\prime}\right|=q-j} \operatorname{det}\left(M_{i i^{\prime}}\right)_{i \in I, i^{\prime} \in I^{\prime}} \mathrm{d} x_{I} \wedge \mathrm{d} \bar{x}_{I^{\prime}}\right) \sum_{\substack{1 \leq k \leq q-1 \\ 0 \leq l \leq N}} \mathcal{C}_{l l}^{k k}(u)(z) .
$$

As a result of all that, since $K_{1}=K_{2}$, we obtain the following expression

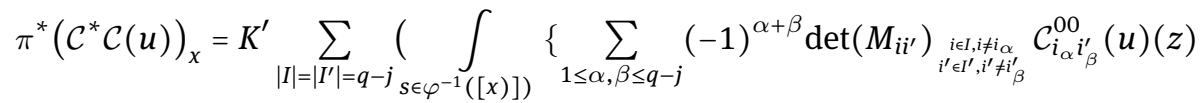

$$
\begin{aligned}
& \left.\left.+\operatorname{det}\left(M_{i i^{\prime}}\right)_{i \in I, i^{\prime} \in I^{\prime}} \sum_{\substack{1 \leq k \leq q-1 \\
0 \leq 1 \leq N}} \mathcal{C}_{l l}^{k k}(u)(z)\right\} \Omega_{s}^{d}\right) \mathrm{d} x_{I} \wedge \mathrm{d} \bar{x}_{I^{\prime}}
\end{aligned}
$$

where $\left.s \in \varphi^{-1}([x)]\right)$ is written $s=\operatorname{vect}\left(x, z^{1}, \ldots, z^{q-1}\right)$ and where $K^{\prime}=\frac{K_{1}}{K}=\frac{K_{2}}{K}$ is a constant.

Let $F_{I, I^{\prime}}$ be the smooth function on $G(q, V)$ defined by

$$
\begin{array}{r}
F_{I, I^{\prime}}(s)=F_{I, I^{\prime}}\left(\operatorname{vect}\left(x, z^{1}, \ldots, z^{q-1}\right)\right)=\sum_{1 \leq \alpha, \beta \leq q-j}(-1)^{\alpha+\beta} \operatorname{det}\left(M_{i i^{\prime}}\right)_{\substack{i \in I, i \neq i \alpha \\
i^{\prime} \in I^{\prime}, i^{\prime} \neq i_{\beta}^{\prime}}} \mathcal{C}_{i_{\alpha} i_{\beta}^{\prime}}^{00}(u)(z)+ \\
\operatorname{det}\left(M_{i i^{\prime}}\right)_{i \in I, i^{\prime} \in I^{\prime}} \sum_{\substack{1 \leq k \leq q-1 \\
0 \leq l \leq N}} \mathcal{C}_{l l}^{k k}(u)(z) .
\end{array}
$$

Then the expression in coordinates of $\mathcal{C}^{*} \mathcal{C}(u)$ is given by the following result.

Lemma 1. For all $x \in \mathbb{C}^{N+1}=V$ such that $x \neq 0$, we have

$$
\begin{aligned}
\pi^{*}\left(\mathcal{C}^{*} \mathcal{C}(u)\right)_{x} & =K^{\prime} \sum_{|I|=\left|I^{\prime}\right|=q-j} \mathrm{~d} x_{I} \wedge \mathrm{d} \bar{x}_{I^{\prime}} \int_{\left.s \in \varphi^{-1}([x)]\right)} F_{I, I^{\prime}} \Omega^{d} \\
& \left.=K^{\prime} \sum_{|I|=\left|I^{\prime}\right|=q-j} \mathrm{~d} x_{I} \wedge \mathrm{d} \bar{x}_{I^{\prime}}\left({ }^{t} \mathcal{R}_{q-1}\right)\left(F_{I, I^{\prime}}\right)([x)]\right) .
\end{aligned}
$$

where ${ }^{t} \mathcal{R}_{q-1}$ is the dual transform of the projective $(q-1)$-dimensional Radon transform $\mathcal{R}_{q-1}$. 


\subsection{Expression of the $F_{I, I^{\prime}}$ after a change of basis}

First we express the $F_{I, I^{\prime}}$ by means of the transforms $\tilde{u}_{K, K^{\prime}}$. Note that the relation

$$
\begin{aligned}
& \mathcal{C}_{n l}^{k m}(u)(z)=\mathcal{C}(u)_{s}\left(\frac{\mathrm{d}}{\mathrm{d} t} \operatorname{vect}\left(z^{0}, \ldots, z^{k-1}, z^{k}+t e_{n}, z^{k+1}, \ldots, z^{q-1}\right)_{\mid t=0},\right. \\
& \left.\overline{\frac{\mathrm{d}}{\mathrm{d} t} \operatorname{vect}\left(z^{0}, \ldots, z^{m-1}, z^{m}+t e_{l}, z^{m+1}, \ldots, z^{q-1}\right)_{\mid t=0}}\right)
\end{aligned}
$$

implies

$$
\mathcal{C}_{n l}^{k k}(u)(z)=\mathcal{C}_{n l}^{00}(u)\left(z^{k}, z^{1}, \ldots, z^{k-1}, z^{0}, z^{k+1}, \ldots, z^{q-1}\right) .
$$

On another hand, with $\left(a_{k, m}\right)_{1 \leq k, m \leq q-1} \in G L_{q-1}(\mathbb{C}) \subset G L_{q}(\mathbb{C})$, set

$$
g\left(z^{0}, z^{1}, \ldots, z^{q-1}\right)=\left(z^{0}, \sum_{1 \leq k \leq q-1} a_{k, 1} z^{k}, \ldots, \sum_{1 \leq k \leq q-1} a_{k, q-1} z^{k}\right) .
$$

Then we have $\tau(g(z))=\tau(z)$ thus

$$
g^{*} \sum_{\substack{0 \leq k, m \leq-1-1 \\ 0 \leq n, l \leq N}} \mathcal{C}_{n l}^{k m}(u) \mathrm{d} z_{n}^{k} \wedge \mathrm{d} \bar{z}_{l}^{m}=\sum_{\substack{0 \leq k, m \leq q-1 \\ 0 \leq n, l \leq N}} \mathcal{C}_{n l}^{k m}(u) \mathrm{d} z_{n}^{k} \wedge \mathrm{d} \bar{z}_{l}^{m} .
$$

We obtain

$$
\sum_{0 \leq n, l \leq N} \mathcal{C}_{n l}^{00}(u)(g(z)) \mathrm{d} z_{n}^{0} \wedge \mathrm{d} \bar{z}_{l}^{0}+\ldots=\sum_{0 \leq n, l \leq N} \mathcal{C}_{n l}^{00}(u)(z) \mathrm{d} z_{n}^{0} \wedge \mathrm{d} \bar{z}_{l}^{0}+\ldots
$$

thus the invariance relation $\mathcal{C}_{n l}^{00}(u)(g(z))=\mathcal{C}_{n l}^{00}(u)(z)$.

Now we can express the functions $F_{I, I^{\prime}}$ for $|I|=\left|I^{\prime}\right|=q-j$, in terms of the Radon transforms $\mathcal{R}_{q-1}\left(f_{K, K^{\prime}}\right)$ for $|K|=\left|K^{\prime}\right|=q$.

First

$$
\begin{aligned}
& \sum_{1 \leq \alpha, \beta \leq q-j}(-1)^{\alpha+\beta} \operatorname{det}\left(M_{i i^{\prime}}{\substack{i \in I, i, i i_{\alpha} \\
i^{\prime} \in I^{\prime}, i^{\prime} \neq i_{\beta}^{\prime}}}_{\substack{i_{\alpha} i_{\beta}^{\prime} \\
0}}^{00}(u)(z)\right.
\end{aligned}
$$

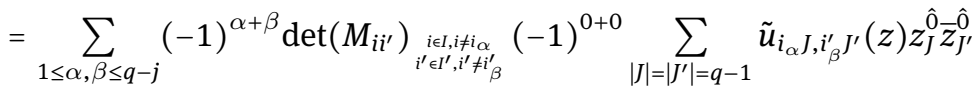

$$
\begin{aligned}
& =\sum_{|K|=\left|K^{\prime}\right|=q} \tilde{u}_{K, K^{\prime}}(z) \sum_{i_{\alpha} \in K, i_{\beta}^{\prime} \in K^{\prime}}(-1)^{\alpha+\beta}(-1)^{\alpha_{0}+\beta_{0}} \operatorname{det}\left(M_{i i^{\prime}}\right) \underset{\substack{i \in I^{\prime}, i \neq i_{i} \\
i^{\prime} \in I^{\prime}, i^{\prime} \neq i_{\beta}^{\prime}}}{\hat{0}} z_{K \backslash\left\{i_{\alpha}\right\}}^{\hat{0}} \bar{z}_{K^{\prime} \backslash\left\{i_{\beta}^{\prime}\right\}}^{\hat{0}}
\end{aligned}
$$

where $\alpha_{0}$ and $\beta_{0}$ are such that $i_{\alpha}=k_{\alpha_{0}}$ and $i_{\beta}^{\prime}=k_{\beta_{0}}^{\prime}$ when $K=\left(k_{1}, \ldots, k_{q}\right)$ with $0 \leq k_{1}<\ldots<k_{q} \leq N$ and $K^{\prime}=\left(k_{1}^{\prime}, \ldots, k_{q}^{\prime}\right)$ with $0 \leq k_{1}^{\prime}<\ldots<k_{q}^{\prime} \leq N$.

Then

$$
\begin{aligned}
& \operatorname{det}\left(M_{i i^{\prime}}\right)_{i \in I, i^{\prime} \in I^{\prime}} \sum_{\substack{1 \leq k \leq q-1 \\
0 \leq 1 \leq N}} \mathcal{C}_{l l}^{k k}(u)(z) \\
= & \sum_{0 \leq l \leq N} \sum_{|J|=\left|J^{\prime}\right|=q-1} \tilde{u}_{l J, l J^{\prime}}(z) \sum_{1 \leq k \leq q-1} \operatorname{det}\left(M_{i i^{\prime}}\right)_{i \in I, i^{\prime} \in I^{\prime}} z_{J}^{\hat{k}} \bar{z}_{J^{\prime}}^{\hat{k}} \\
= & \sum_{|K|=\left|K^{\prime}\right|=q} \tilde{u}_{K, K^{\prime}}(z) \sum_{l \in K \cap K^{\prime}}(-1)^{\alpha_{0}+\beta_{0}} \sum_{1 \leq k \leq q-1} \operatorname{det}\left(M_{i i^{\prime}}\right)_{i \in I, i^{\prime} \in I^{\prime}} z_{K \backslash\{l\}}^{\hat{k}^{k}} \bar{z}_{K^{\prime} \backslash\{l\}}^{\hat{k}}
\end{aligned}
$$

where $\alpha_{0}$ and $\beta_{0}$ are such that $l=k_{\alpha_{0}}=k_{\beta_{0}}^{\prime}$ when $K=\left(k_{1}, \ldots, k_{q}\right)$ with $0 \leq k_{1}<\ldots<k_{q} \leq N$ and $K^{\prime}=\left(k_{1}^{\prime}, \ldots, k_{q}^{\prime}\right)$ with $0 \leq k_{1}^{\prime}<\ldots<k_{q}^{\prime} \leq N$.

With all that together, we conclude that

$$
F_{I, I^{\prime}}(s)=\sum_{|K|=\left|K^{\prime}\right|=q} \mathcal{R}_{q-1}\left(f_{K, K^{\prime}}\right)(s) c_{K, K^{\prime}}^{I, I^{\prime}}(s)
$$

where the coefficients $c_{K, K^{\prime}}^{I, I^{\prime}}(s)$ are independent of the smooth differential $(q, q)$-form $u$. 
Set the matrix $C(s)=\left(c_{K, K^{\prime}}^{I, I^{\prime}}(s)\right)_{I I^{\prime}, K K^{\prime}}$. Note here that $\left(K, K^{\prime}\right)$ belongs to a set with $\left(\begin{array}{c}N+1 \\ q\end{array}\right)^{2}$ elements, while $\left(I, I^{\prime}\right)$ belongs to a set with $\left(\begin{array}{c}N+1 \\ q-j\end{array}\right)^{2}=\left(\begin{array}{c}N+1 \\ q\end{array}\right)^{2}$ in both cases $j=0$ or $j=2 q-N-1$.

When $g \in U(N+1)$ is an element of the unitary group of $V=\mathbb{C}^{N+1}$, we can express $C(g(s))$ in terms of $g$ and $C(s)$ by means of the formula

$$
C(g(s))={ }^{t} Q(g)^{-1} C(s)^{t} Q(g) \Leftrightarrow{ }^{t} Q(g) C(g(s))=C(s)^{t} Q(g)
$$

with the matrix $Q(g)=\left(\operatorname{det}\left(g_{k, l}\right)_{\substack{k \in K \\ l \in L}} \operatorname{det}\left(\bar{g}_{k^{\prime}, l^{\prime}}\right)_{\substack{k^{\prime} \in K^{\prime} \\ l^{\prime} \in I^{\prime}}}\right)_{K K^{\prime}, L L^{\prime}}$.

For the proof of this invariance property, let $\lambda_{K}$ be the Plücker coordinates of $\left(\lambda^{0}, \ldots, \lambda^{q-1}\right) \in\left(\mathbb{C}^{N+1}\right)^{q}$ defined by

$$
\lambda^{0} \wedge \ldots \wedge \lambda^{q-1}=\sum_{|K|=q} \lambda_{K} e_{K}
$$

This property is a consequence of the fact that the coefficients $c_{K, K^{\prime}}^{I, I^{\prime}}(s)$ can be calculated by means of the relation

$$
\begin{aligned}
& \alpha_{q} \Delta(z) \sum_{|I|=\left|I^{\prime}\right|=q-j|K|=\left|K^{\prime}\right|=q} \bar{\lambda}_{K} \lambda_{K^{\prime}} c_{K, K^{\prime}}^{I, I^{\prime}}(s) \mathrm{d} x_{I} \wedge \mathrm{d} \bar{x}_{I^{\prime}} \\
& =D_{1} p_{s^{\perp}}^{*}\left(i \partial \bar{\partial}\left(\|x\|^{2}\right)\right)^{q-j-1} \wedge \partial \bar{\partial}\left(\left|\left\langle x \wedge z^{1} \wedge \ldots \wedge z^{q-1} \mid \lambda^{0} \wedge \ldots \wedge \lambda^{q-1}\right\rangle\right|^{2}\right) \\
& +D_{2} p_{s^{\perp}}^{*}\left(i \partial \bar{\partial}\left(\|x\|^{2}\right)\right)^{q-j} \sum_{1 \leq k \leq q-1} \operatorname{trace}\left(\left|\left\langle x \wedge z^{0} \wedge \ldots \wedge \widehat{z^{k}} \wedge \ldots \wedge z^{q-1} \mid \lambda^{0} \wedge \ldots \wedge \lambda^{q-1}\right\rangle\right|^{2}\right)
\end{aligned}
$$

with $D_{1}=(-1)^{q-j-1}\left(i^{(q-j-1)^{2}}(q-j-1) !\right)^{-1}$ and $D_{2}=\left(i^{(q-j)^{2}}(q-j) !\right)^{-1}$.

Since the differential $(1,1)$-form $\Omega$ is invariant by $U(N+1)$, we have also $g^{*} \pi^{*} \mathcal{C}^{*} \mathcal{C}(u)=\pi^{*} \mathcal{C}^{*} \mathcal{C}\left(g^{*} u\right)$. But because ${ }^{t} \mathcal{R}_{q-1}$ is not injective (see [14]), we cannot deduce directly from the previous relation the invariance property for the matrix $C(s)$.

Note that $Q(g)$ is the matrix relatively to the basis $\left(e_{L} \wedge \bar{e}_{L^{\prime}}\right)_{L L^{\prime}}$ of $\wedge^{q} V \otimes \wedge^{q} \bar{V}$ of the unitary operator $\wedge^{q} g \otimes \wedge^{q} \bar{g}$, therefore $Q(g)$ is unitary.

For $|J|=\left|J^{\prime}\right|=q-j$, set

$$
G_{J, J^{\prime}}(g)=\sum_{|I|=\left|I^{\prime}\right|=q-j} Q_{I I^{\prime}, J J^{\prime}}(g) F_{I, I^{\prime}}(g(s))=\sum_{|L|=\left|L^{\prime}\right|=q} c_{L, L^{\prime}}^{J, J^{\prime}}(s) \varphi_{L, L^{\prime}}(g)
$$

thanks to (16), with

$$
\varphi_{L, L^{\prime}}(g)=\sum_{|K|=\left|K^{\prime}\right|=q} \mathcal{R}_{q-1}\left(f_{K, K^{\prime}}\right)(g(s)) Q_{K K^{\prime}, L L^{\prime}}(g) .
$$

Let the $h_{K, K^{\prime}}$ be smooth functions on $\mathbb{P}_{N}=P(V)$ and set

$$
\psi_{L, L^{\prime}}(g)=\sum_{|K|=\left|K^{\prime}\right|=q} \mathcal{R}_{q-1}\left(h_{K, K^{\prime}}\right)(g(s)) Q_{K K^{\prime}, L L^{\prime}}(g) .
$$

Since $Q(g)$ is unitary, we have

$$
\begin{aligned}
\sum_{|L|=\left|L^{\prime}\right|=q}\left(\psi_{L, L^{\prime}} \bar{G}_{L, L^{\prime}}\right)(g) & =\sum_{|I|=\left|I^{\prime}\right|=q-j|K|=\left|K^{\prime}\right|=q}\left(\mathcal{R}_{q-1}\left(h_{K, K^{\prime}}\right) \bar{F}_{I, I^{\prime}}\right)(g(s))\left(Q(g) Q^{*}(g)\right)_{K K^{\prime}, I^{\prime}} \\
& =\sum_{|I|=\left|I^{\prime}\right|=q-j}\left(\mathcal{R}_{q-1}\left(h_{I, I^{\prime}}\right) \bar{F}_{I, I^{\prime}}\right)(g(s)) .
\end{aligned}
$$

\subsection{Determination of ker $\mathcal{C}^{*} \mathcal{C}$}

First we determine the system of linear P.D.E. satisfied by the functions $\psi_{L, L^{\prime}}(g) \alpha_{q}^{-1} \Delta(g(z))^{-1}$ of $g$. With $s=\operatorname{vect}\left(z^{0}, \ldots, z^{q-1}\right)$, we have

$$
\varphi_{L, L^{\prime}}(g) \alpha_{q}^{-1} \Delta(g(z))^{-1}
$$




$$
\begin{aligned}
& =\sum_{|K|=\left|K^{\prime}\right|=q} \tilde{u}_{K, K^{\prime}}(g(z)) Q_{K K^{\prime}, L L^{\prime}}(g) \\
& =\int_{t \in \mathbb{S}^{2 q-1}}\left\langle\left(\pi^{*} u\right)_{t \cdot g(z)},\left(\bigwedge^{q} g \otimes \bigwedge^{q} \bar{g}\right)\left(e_{L} \wedge \bar{e}_{L^{\prime}}\right)\right\rangle \Phi(t) \\
& =\int_{t \in \mathbb{S}^{2 q-1}}\left\langle\left(\pi^{*}\left(g^{*} u\right)\right)_{t \cdot z}, e_{L} \wedge \bar{e}_{L^{\prime}}\right\rangle \Phi(t) .
\end{aligned}
$$

When $z=\left(z^{0}, \ldots, z^{q-1}\right)$ is fixed, the map

$$
g \rightarrow \int_{t \in \mathbb{S}^{2 q-1}}\left(\pi^{*}\left(g^{*} u\right)\right)_{t \cdot z} \Phi(t) \in \bigwedge^{q, q} V^{*}
$$

is an integral transform of $u$. By complexifying i.e. by taking $g \in G L(N+1, \mathbb{C})$, this map has the same expression in $g$ and satisfies a system of linear partial differential equations in $g$.

As a consequence, when the functions $h_{K, K^{\prime}}$ vary in $\mathcal{C}^{\infty}\left(\mathbb{P}_{N}\right)$, the functions $\psi_{L, L^{\prime}}$ vary in a vector space $\mathcal{E}$ of smooth functions in $g$, characterized by a system of linear partial differential equations, which does not depend on $\left(L, L^{\prime}\right)$.

Lemma 2. If $F_{I, I^{\prime}} \in \operatorname{ker}\left({ }^{t} \mathcal{R}_{q-1}\right)$ for all $|I|=\left|I^{\prime}\right|=q-j$, then $F_{I, I^{\prime}}=0$ for all $|I|=\left|I^{\prime}\right|=q-j$.

Proof. If $F_{I, I^{\prime}} \in \operatorname{ker}\left({ }^{t} \mathcal{R}_{q-1}\right)=\left(\operatorname{im} \mathcal{R}_{q-1}\right)^{\perp}$ for all $|I|=\left|I^{\prime}\right|=q-j$, then $G_{L, L^{\prime}}$ is orthogonal to each $\psi_{L, L^{\prime}}$ for all $|L|=\left|L^{\prime}\right|=q$, thus $G_{L, L^{\prime}} \in \mathcal{E}^{\perp}$. But since the $G_{J, J^{\prime}}$ decompose on the $\varphi_{L, L^{\prime}}$ with constant coefficients, we have $G_{J, J^{\prime}} \in \mathcal{E}$, therefore $G_{J, J^{\prime}}=0$. Since the matrix $Q(g)$ is invertible, this implies $F_{I, I^{\prime}}=0$ for all $|I|=\left|I^{\prime}\right|=q-j$.

In other words, $0=\mathcal{C}^{*} \mathcal{C}(u)$ implies

$$
0=\sum_{|I|=\left|I^{\prime}\right|=q-j} F_{I, I^{\prime}}(\tau(z)) \mathrm{d} x_{I} \wedge \mathrm{d} \bar{x}_{I^{\prime}}
$$

which is equivalent to

$$
\begin{array}{r}
0=(q-j) p_{s^{\perp}}^{*}\left(i \partial \bar{\partial}\left(\|x\|^{2}\right)\right)^{q-j-1} \wedge \sum_{0 \leq n, l \leq N} \mathcal{C}_{n l}^{00}(u)(z) i \mathrm{~d} x_{n} \wedge \mathrm{d} \bar{x}_{l} \\
+p_{s^{\perp}}^{*}\left(i \partial \bar{\partial}\left(\|x\|^{2}\right)\right)^{q-j} \sum_{\substack{1 \leq k \leq q-1 \\
0 \leq l \leq N}} \mathcal{C}_{l l}^{k k}(u)(z) .
\end{array}
$$

That is to say $0=p_{S^{\perp}}^{*}\left(i \partial \bar{\partial}\left(\|x\|^{2}\right)\right)^{q-j-1} \wedge \theta$ with the differential $(1,1)$-form $\theta$ in $V=\mathbb{C}^{N+1}$ defined by

$$
\theta=\sum_{0 \leq n, l \leq N}\left\{(q-j) \mathcal{C}_{n l}^{00}(u)(z)+M_{n l} \sum_{\substack{1 \leq k \leq q-1 \\ 0 \leq l^{\prime} \leq N}} \mathcal{C}_{l^{\prime} l^{\prime}}^{k k}(u)(z)\right\} i \mathrm{~d} x_{n} \wedge \mathrm{d} \bar{x}_{l} .
$$

We now prove that necessarily $u=0$.

Let $b_{q}, \ldots, b_{N}$ be an orthonormal basis of $s^{\perp}$. Denote by $b_{l}^{*} \in V^{*}$ the linear form defined by $b_{l}^{*}(x)=\left\langle x \mid b_{l}\right\rangle$. Since $d(x, s)^{2}=\sum_{q \leq l \leq N}\left|\left\langle x \mid b_{l}\right\rangle\right|^{2}$, we have

$$
\left(i \partial \bar{\partial}\left(d(x, s)^{2}\right)\right)^{q-j-1}=\left(i \sum_{q \leq l \leq N} b_{l}^{*} \wedge \bar{b}_{l}^{*}\right)^{q-j-1}=D \sum_{|I|=q-j-1} b_{I}^{*} \wedge \bar{b}_{I}^{*}
$$

where $I=\left(i_{1}, \ldots, i_{q-j-1}\right)$ with $q \leq i_{1}<\ldots<i_{q-j-1} \leq N$ and where $D=i^{(q-j-1)^{2}}(q-j-1)$ !.

Let $b_{0}, \ldots, b_{q-1}$ be an orthonormal basis of $s=\operatorname{vect}\left(z^{0}, \ldots, z^{q-1}\right)$. Writing $\theta=\sum_{0 \leq n, l \leq N} \check{\theta}_{n l} b_{n}^{*} \wedge \bar{b}_{l}^{*}$ in the basis $b_{l}^{*}$, we have

$$
0=\sum_{|I|=q-j-1} b_{I}^{*} \wedge \bar{b}_{I}^{*} \wedge \sum_{0 \leq n, l \leq N} \check{\theta}_{n l} b_{n}^{*} \wedge \bar{b}_{l}^{*}=(-1)^{q-j-1} \sum_{|I|=q-j-1} \sum_{n, l \notin I} \check{\theta}_{n l} b_{n I}^{*} \wedge \bar{b}_{l I}^{*}
$$

We set $J=\left\{n, i_{1}, \ldots, i_{q-j-1}\right\}$ and $J^{\prime}=\left\{l, i_{1}, \ldots, i_{q-j-1}\right\}$ in such a way that card $\left(J \cap J^{\prime}\right) \geq q-j-1$. Then $\operatorname{card}\left(J \cap J^{\prime}\right)=q-j-1 \Leftrightarrow n \neq l$ and $J=J^{\prime} \Leftrightarrow n=l$. 
In the first case $I=J \cap J^{\prime}$ and $\{n\}=J \backslash J \cap J^{\prime}$ while $\{l\}=J^{\prime} \backslash J \cap J^{\prime}$ : we obtain $\check{\theta}_{n l}=0=\theta\left(b_{n}, \bar{b}_{l}\right)$ if $n \neq l$. On another hand we have

$$
\sum_{|I|=q-j-1} \sum_{l \notin I} \check{\theta}_{l l} b_{l I}^{*} \wedge \bar{b}_{l I}^{*}=\sum_{|I|=q-j-1} \sum_{\substack{l \neq l \\ l<q}} \check{\theta}_{l l} b_{l I}^{*} \wedge \bar{b}_{l I}^{*}+\sum_{|J|=q-j}\left(\sum_{l \in J} \check{\theta}_{l l}\right) b_{J}^{*} \wedge \bar{b}_{J}^{*}
$$

where $J=\left(i_{1}, \ldots, i_{q-j}\right)$ with $q \leq i_{1}<\ldots<i_{q-j} \leq N$. In the second case, we obtain $\check{\theta}_{l l}=0$ if $0 \leq l \leq q-1$, while $\sum_{l \in J} \check{\theta}_{l l}=0$ for all such $J$. It remains $N-q+1$ unknown variables $\check{\theta}_{l l}$ satisfying $\left(\begin{array}{c}N-q+1 \\ q-j\end{array}\right)$ relations.

If $j=0 \Leftrightarrow q \leq N-q+1$, we have $\left(\begin{array}{c}N-q+1 \\ q-j\end{array}\right)=\left(\begin{array}{c}N-q+1 \\ q\end{array}\right) \geq\left(\begin{array}{c}N-q+1 \\ 1\end{array}\right)$ i.e. $\geq N-q+1$. The number of equations being bigger, we obtain $\theta=0$.

If $j=2 q-N-1 \Leftrightarrow q \geq N-q+1$, we have $\theta\left(b_{n}, \bar{b}_{l}\right)=0$ for all $(n, l)$ except for $n=l \geq q$, for which we only have $\sum_{q \leq l \leq N} \theta\left(b_{l}, \bar{b}_{l}\right)=0$.

Recall that

$$
\begin{aligned}
& \frac{1}{i} \theta\left(b_{n}, \bar{b}_{l}\right)=\int_{t \in \mathbb{S}^{2 q-1}}\left\{(q-j)\left(\pi^{*} u\right)_{t \cdot z}\left(b_{n}, z^{1}, \ldots, z^{q-1}, \bar{b}_{l}, \bar{z}^{1}, \ldots, \bar{z}^{q-1}\right)+{ }^{t} b_{n}\left(M_{i, i^{\prime}}\right) \bar{b}_{l}\right. \\
& \left.\cdot \sum_{\substack{1 \leq k \leq q-1 \\
0 \leq l^{\prime} \leq N}}\left(\pi^{*} u\right)_{t \cdot z}\left(e_{l^{\prime}}, z^{0}, \ldots, z^{k-1}, z^{k+1}, \ldots, z^{q-1}, \bar{e}_{l^{\prime}}, \bar{z}^{0}, \ldots, \bar{z}^{k-1}, \bar{z}^{k+1}, \ldots, \bar{z}^{q-1}\right)\right\} \Phi(t) .
\end{aligned}
$$

Thus we obtain the condition

$$
\begin{aligned}
& 0=(q-j)\left(\pi^{*} u\right)_{x}\left(b_{n}, z^{1}, \ldots, z^{q-1}, \bar{b}_{l}, \bar{z}^{1}, \ldots, \bar{z}^{q-1}\right) \\
& +{ }^{t} b_{n}\left(M_{i, i^{\prime}}\right) \bar{b}_{l} \sum_{\substack{1 \leq k \leq q-1 \\
0 \leq l^{\prime} \leq N}}\left(\pi^{*} u\right)_{x}\left(e_{l^{\prime}}, z^{0}, \ldots, z^{k-1}, z^{k+1}, \ldots, z^{q-1}, \bar{e}_{l^{\prime}}, \bar{z}^{0}, \ldots, \bar{z}^{k-1}, \bar{z}^{k+1}, \ldots, \bar{z}^{q-1}\right)
\end{aligned}
$$

where $x=t \cdot z=t_{1} z^{0}+\ldots+t_{q} z^{q-1}$, for every $t=\left(t_{1}, \ldots, t_{q}\right) \in \mathbb{S}^{2 q-1}$ and for every $z=\left(z^{0}, \ldots, z^{q-1}\right) \epsilon$ $\left(\mathbb{C}^{N+1}\right)^{q}$. We have denoted by ${ }^{t} b_{n}\left(M_{i, i^{\prime}}\right) \bar{b}_{l}=\left(\partial \bar{\partial}\left(d(x, s)^{2}\right)\right)\left(b_{n} \wedge \bar{b}_{l}\right)$ the value at $\left(b_{n}, b_{l}\right)$ of the Hermitian form associated to the matrix $\left(M_{i, i^{\prime}}\right)_{i i^{\prime}}$.

By taking $z^{0}=x$ and by using that the radial contractions of $\left(\pi^{*} u\right)_{x}$ are equal to 0 , it remains

$$
\left(\pi^{*} u\right)_{x}\left(b_{n}, z^{1}, \ldots, z^{q-1}, \bar{b}_{l}, \bar{z}^{1}, \ldots, \bar{z}^{q-1}\right)=0
$$

for all $n, l$, therefore $\left(\pi^{*} u\right)_{x}\left(e_{n}, z^{1}, \ldots, z^{q-1}, \bar{e}_{l}, \bar{z}^{1}, \ldots, \bar{z}^{q-1}\right)=0$ for all $x$ and all $z^{1}, \ldots, z^{q-1}$. We can assume the differential $(q, q)$-form $u$ real, then we obtain $\left(\pi^{*} u\right)_{x}=0$ i.e. $u=0$.

If $j=2 q-N-1$, the relation (17) is valid for $n \neq l$ and for $n=l<q$. Therefore we can write

$$
\left(\pi^{*} u\right)_{x}\left(., z^{1}, \ldots, z^{q-1}, ., \bar{z}^{1}, \ldots, \bar{z}^{q-1}\right)=\sum_{q \leq l \leq N} \lambda_{l}\left(x, z^{1}, \ldots, z^{q-1}\right) b_{l}^{*} \wedge \bar{b}_{l}^{*}
$$

with functions $\lambda_{l}\left(x, z^{1}, \ldots, z^{q-1}\right)$ of $x, z^{1}, \ldots, z^{q-1}$. But the family $\left(b_{q}, \ldots, b_{N}\right)$ is any orthonormal basis of $\operatorname{vect}\left(x, z^{1}, \ldots, z^{q-1}\right)^{\perp}$, thus the function $\lambda_{l}\left(x, z^{1}, \ldots, z^{q-1}\right)=\lambda\left(x, z^{1}, \ldots, z^{q-1}\right)$ does not depend on $l$. The relation (18) becomes

$$
\left(\pi^{*} u\right)_{x}\left(., z^{1}, \ldots, z^{q-1}, ., \bar{z}^{1}, \ldots, \bar{z}^{q-1}\right)=\lambda\left(x, z^{1}, \ldots, z^{q-1}\right) \partial \bar{\partial}\left(d(., s)^{2}\right),
$$

which gives $\theta\left(b_{l}, \bar{b}_{l}\right)$ independent of $q \leq l \leq N$. We obtain that $\theta\left(b_{l}, \bar{b}_{l}\right)=0$ thus $\theta=0$ and that the same conclusion is valid as for the case $j=0$ i.e. $u=0$.

\section{Theorem 1. The integral transformation}

$$
\mathcal{C}^{*} \mathcal{C}:\left\{\text { currents of bidegree }(q, q) \text { on } \mathbb{P}_{N}\right\} \rightarrow\left\{\text { currents of bidegree }(q-j, q-j) \text { on } \mathbb{P}_{N}\right\}
$$
is injective. 
Remark 1. If $u$ is a smooth differential $(q, q)$-form on $\mathbb{P}_{N}$ satisfying

$$
0=\sum_{|I|=|J|=q-1} \tilde{u}_{n I, l J}(z) z_{I}^{\hat{0}} \bar{z}_{J}^{\hat{0}} \Leftrightarrow 0=\int_{t \in \mathbb{S}^{2 q-1}}\left(\pi^{*} u\right)_{t \cdot z}\left(e_{n}, z^{1}, \ldots, z^{q-1}, \bar{e}_{l}, \bar{z}^{1}, \ldots, \bar{z}^{q-1}\right) \Phi(t)
$$

on $\left(\mathbb{C}^{N+1}\right)^{q}$ for all $0 \leq n, l \leq N$, then $u=0$.

In effect, for $0 \leq n, l \leq N$, we define a smooth differential $(q-1, q-1)$-form $\varphi=\varphi_{n, l}$ on $\mathbb{P}_{N}=P(V)$ by

$$
\varphi_{[x]}=\|x\|^{2} u_{[x]}\left(\mathrm{d} \pi_{x}\left(e_{n}\right), \ldots, \overline{\mathrm{d} \pi_{x}\left(e_{l}\right)}, \ldots\right)
$$

for all $x \in V \backslash\{0\}$. In other words, we have

$$
\left(\pi^{*} u\right)_{x}\left(e_{n}, z^{1}, \ldots, z^{q-1}, \bar{e}_{l}, \bar{z}^{1}, \ldots, \bar{z}^{q-1}\right)=\|x\|^{-2}\left(\pi^{*} \varphi\right)_{x}\left(z^{1}, \ldots, z^{q-1}, \bar{z}^{1}, \ldots, \bar{z}^{q-1}\right)
$$

with

$$
(-1)^{q-1} q^{2}\left(\pi^{*} u\right)_{x}=\|x\|^{-2} \sum_{0 \leq n, l \leq N}\left(\pi^{*} \varphi_{n, l}\right)_{x} \wedge \mathrm{d} x_{n} \wedge \mathrm{d} \bar{x}_{l} .
$$

Let us now express the Chow transform $\mathcal{C}(\varphi)$ i.e. let us calculate

$$
\int_{P(\tau(z))} \varphi
$$

with $\tau(z)=\operatorname{vect}\left(z^{0}, \ldots, z^{q-1}\right) \in G(q, V)$. To this end, we introduce the function $f \in \mathcal{C}^{\infty}(P(\tau(z)))$ such that

$$
\varphi_{\mid P(\tau(z))}=f \omega_{\mid P(\tau(z))}^{q-1} .
$$

For $x \in \tau(z) \backslash\{0\}$ and $0 \leq k \leq q-1$, we have $\mathrm{d} \pi_{x}\left(z^{k}\right) \in T_{[x]} P(\tau(z))$ and so

$$
\begin{array}{r}
\varphi_{[x]}\left(\mathrm{d} \pi_{x}\left(z^{1}\right), \ldots, \mathrm{d} \pi_{x}\left(z^{q-1}\right), \overline{\mathrm{d} \pi_{x}\left(z^{1}\right)}, \ldots, \overline{\mathrm{d} \pi_{x}\left(z^{q-1}\right)}\right) \\
=f([x]) \omega_{[x]}^{q-1}\left(\mathrm{~d} \pi_{x}\left(z^{1}\right), \ldots, \mathrm{d} \pi_{x}\left(z^{q-1}\right), \overline{\mathrm{d} \pi_{x}\left(z^{1}\right)}, \ldots, \overline{\mathrm{d} \pi_{x}\left(z^{q-1}\right)}\right) .
\end{array}
$$

With $C=\frac{i^{(q-1)^{2}}(q-1) !}{(2 \pi)^{q-1}}$, we use that

$$
\begin{aligned}
& \omega_{[x]}^{q-1}\left(\mathrm{~d} \pi_{x}\left(z^{1}\right), \ldots, \mathrm{d} \pi_{x}\left(z^{q-1}\right), \overline{\mathrm{d} \pi_{x}\left(z^{1}\right)}, \ldots, \overline{\mathrm{d} \pi_{x}\left(z^{q-1}\right)}\right) \\
= & C\|x\|^{-2 q+2}\left\|\left(z^{1}-\frac{\left\langle z^{1} \mid x\right\rangle}{\|x\|^{2}} x\right) \wedge \ldots \wedge\left(z^{q-1}-\frac{\left\langle z^{q-1} \mid x\right\rangle}{\|x\|^{2}} x\right)\right\|^{2} \\
= & C\|x\|^{-2 q}\left|\left\langle x \mid z^{0}\right\rangle\right|^{2}
\end{aligned}
$$

by assuming here that $\left(z^{0}, \ldots, z^{q-1}\right)$ is an orthonormal basis of $\tau(z)$. First we obtain

$$
\begin{aligned}
\int_{P(\tau(z))} \frac{\left|\left\langle x \mid z^{0}\right\rangle\right|^{2}}{\|x\|^{2}} \varphi & =\int_{P(\tau(z))} \frac{\left|\left\langle x \mid z^{0}\right\rangle\right|^{2}}{\|x\|^{2}} f \omega_{\mid P(\tau(z))}^{q-1} \\
& =\int_{t \in \mathbb{S}^{2 q-1}}\left(\left|\left\langle x \mid z^{0}\right\rangle\right|^{2}\|x\|^{-2 q-2} f([x])\right)_{\mid x=t \cdot z} \Phi(t)
\end{aligned}
$$

which is equal to

$$
\begin{aligned}
& C^{-1} \int_{t \in \mathbb{S}^{2 q-1}}\left\{\|x\|^{-2} \varphi_{[x]}\left(\mathrm{d} \pi_{x}\left(z^{1}\right), \ldots, \mathrm{d} \pi_{x}\left(z^{q-1}\right), \overline{\mathrm{d} \pi_{x}\left(z^{1}\right)}, \ldots, \overline{\mathrm{d} \pi_{x}\left(z^{q-1}\right)}\right)\right\}_{\mid x=t \cdot z} \Phi(t) \\
= & C^{-1} \int_{t \in \mathbb{S}^{2 q-1}}\left(\pi^{*} u\right)_{t \cdot z}\left(e_{n}, z^{1}, \ldots, z^{q-1}, \bar{e}_{l}, \bar{z}^{1}, \ldots, \bar{z}^{q-1}\right) \Phi(t)=0 .
\end{aligned}
$$

As a consequence, we have

$$
\int_{P(\tau(z))} \frac{\left|\left\langle x \mid z^{k}\right\rangle\right|^{2}}{\|x\|^{2}} \varphi=0
$$


for all $0 \leq k \leq q-1$, thus by summing $\int_{P(s)} \varphi=0$ for all $s \in G(q, V)$. This implies $\varphi \in \operatorname{im} \partial+\operatorname{im} \bar{\partial}$. But at the same time, we have

$$
\frac{x_{n^{\prime}} \bar{x}_{l^{\prime}}}{\|x\|^{2}} \varphi \in \operatorname{im} \partial+\operatorname{im} \bar{\partial}
$$

for all $0 \leq n^{\prime}, l^{\prime} \leq N$. As a conclusion, $\varphi_{n, l}=\varphi=0$ and $u=0$ as expected.

\subsection{Integral kernel on $\mathbb{P}_{N} \times \mathbb{P}_{N}$ of the transform $\mathcal{C} * \mathcal{C}$}

We now calculate the integral kernel $\mathcal{K}$ on $\mathbb{P}_{N} \times \mathbb{P}_{N}=P(V) \times P(V)$ of $\mathcal{C}^{*} \mathcal{C}$ i.e. calculate $\mathcal{K}$ such that

$$
\mathcal{C}^{*} \mathcal{C}(u)=\operatorname{pr}_{1 *}\left(\mathcal{K} \wedge \operatorname{pr}_{2}^{*} u\right)
$$

for every smooth differential $(q, q)$-form $u$ on $P(V)=\mathbb{P}_{N}$. To this end we use a base change (see [16])

$$
\begin{aligned}
& \Gamma^{\prime} \stackrel{p_{2}}{\rightarrow} \Gamma \\
& p_{1} \downarrow \downarrow \psi \\
& \Gamma \stackrel{\psi}{\rightarrow} G(q, V)
\end{aligned}
$$

where $\Gamma^{\prime}=\left\{(a, b) \in \Gamma^{2}, \psi(a)=\psi(b)\right\}$ and where $p_{1}: \Gamma^{\prime} \rightarrow \Gamma$ and $p_{2}: \Gamma^{\prime} \rightarrow \Gamma$ are the restrictions of the canonical projections.

Lemma 3. For $u$ a smooth differential $(q, q)$-form on $P(V)$, we have $\psi^{*} \psi_{*} \varphi^{*} u=p_{1 *} p_{2}^{*} \varphi^{*} u$.

Proof. For $([x], s) \in \Gamma$ and $(\eta, \sigma) \in T_{([x], s)} \Gamma \subset T_{[x]} P(V) \times T_{s} G(q, V)$, we have on the one hand

$$
\left(\psi^{*} \psi_{*} \varphi^{*} u\right)_{([x], s)}((\eta, \sigma),(\bar{\eta}, \bar{\sigma}))=\left(\psi_{*} \varphi^{*} u\right)_{s}(\sigma, \bar{\sigma})=\int_{\left[x^{\prime}\right] \in P(s)} u_{\left[x^{\prime}\right]}\left(\xi_{\left[x^{\prime}\right]}, \bar{\xi}_{\left[x^{\prime}\right]}, \ldots\right)
$$

where $\xi_{\left[x^{\prime}\right]} \in T_{\left[x^{\prime}\right]} P(V)$ is such that $\left(\xi_{\left[x^{\prime}\right]}, \sigma\right) \in T_{\left(\left[x^{\prime}\right], s\right)} \Gamma$ for every $\left[x^{\prime}\right] \in P(s)$. But

$$
\Gamma^{\prime}=\left\{\left([x],\left[x^{\prime}\right], s\right) \in P(V) \times P(V) \times G(q, V), \operatorname{vect}\left(x, x^{\prime}\right) \subset s\right\}
$$

and $\left(\eta, \eta^{\prime}, \sigma\right) \in T_{[x]} P(V) \times T_{\left[x^{\prime}\right]} P(V) \times T_{s} G(q, V)$ is in $T_{\left([x],\left[x^{\prime}\right], s\right)} \Gamma^{\prime}$ when $(\eta, \sigma) \in T_{([x], s)} \Gamma$ and $\left(\eta^{\prime}, \sigma\right) \in$ $T_{\left(\left[x^{\prime}\right], s\right)} \Gamma$.

On the other hand, we have

$$
\begin{aligned}
& \left(p_{1 *} p_{2}^{*} \varphi^{*} u\right)_{([x], s)}((\eta, \sigma),(\bar{\eta}, \bar{\sigma})) \\
= & \int_{\left[x^{\prime}\right] \in P(s)} u_{\left[x^{\prime}\right]}\left(\mathrm{d}\left(\varphi \circ p_{2}\right)_{\left([x],\left[x^{\prime}\right], s\right)}\left(\eta, \eta^{\prime}, \sigma\right), \overline{\mathrm{d}\left(\varphi \circ p_{2}\right)_{\left([x],\left[x^{\prime}\right], s\right)}\left(\eta, \eta^{\prime}, \sigma\right)}, \ldots\right) \\
= & \int_{\left[x^{\prime}\right] \in P(s)} u_{\left[x^{\prime}\right]}\left(\eta^{\prime}, \overline{\eta^{\prime}}, \ldots\right)
\end{aligned}
$$

where $\eta^{\prime} \in T_{\left[x^{\prime}\right]} P(V)$ is such that $\left(\eta, \eta^{\prime}, \sigma\right) \in T_{\left([x],\left[x^{\prime}\right], s\right)} \Gamma^{\prime}$ i.e. $\left(\eta^{\prime}, \sigma\right) \in T_{\left(\left[x^{\prime}\right], s\right)} \Gamma$.

Then we use a projection formula

$$
\begin{array}{r}
\psi^{*} \Omega^{d+q-1-j} \wedge \psi^{*} \psi_{*} \varphi^{*} u=\psi^{*} \Omega^{d+q-1-j} \wedge p_{1 *} p_{2}^{*} \varphi^{*} u \\
=p_{1 *}\left(p_{1}^{*} \psi^{*} \Omega^{d+q-1-j} \wedge p_{2}^{*} \varphi^{*} u\right)=p_{1 *}\left(\left(\psi \circ p_{1}\right)^{*} \Omega^{d+q-1-j} \wedge\left(\varphi \circ p_{2}\right)^{*} u\right)
\end{array}
$$

and, with

$$
q: \Gamma^{\prime} \rightarrow P(V) \times P(V)
$$

defined by $q\left([x],\left[x^{\prime}\right], s\right)=\left([x],\left[x^{\prime}\right]\right)$, the commutation formula $\varphi \circ p_{i}=\operatorname{pr}_{i} \circ q$. Thanks to (12), we conclude that

$$
\mathcal{C}^{*} \mathcal{C}(u)=\left(\varphi \circ p_{1}\right)_{*}\left(\left(\psi \circ p_{1}\right)^{*} \Omega^{d+q-1-j} \wedge\left(\varphi \circ p_{2}\right)^{*} u\right)
$$




$$
\begin{aligned}
& =\left(\operatorname{pr}_{1} \circ q\right)_{*}\left(\left(\psi \circ p_{1}\right)^{*} \Omega^{d+q-1-j} \wedge\left(\operatorname{pr}_{2} \circ q\right)^{*} u\right) \\
& =\operatorname{pr}_{1 *}\left\{q_{*}\left(\left(\psi \circ p_{1}\right)^{*} \Omega^{d+q-1-j}\right) \wedge \operatorname{pr}_{2}^{*} u\right\} .
\end{aligned}
$$

Proposition 5. The integral kernel $\mathcal{K}(.,$.$) of \mathcal{C}^{\star} \mathcal{C}$ is given by

$$
\mathcal{K}=q_{*}\left(\left(\psi \circ p_{1}\right)^{*} \Omega^{d+q-1-j}\right)
$$

and it is a differential form of bidegree $(N-j, N-j)$, which is smooth in $(P(V) \times P(V)) \backslash D_{P(V)}$, where $D_{P(V)}$ is the diagonal of $P(V) \times P(V)$.

Proof. The restriction $q: \Gamma^{\prime} \backslash q^{-1}\left(D_{P(V)}\right) \rightarrow(P(V) \times P(V)) \backslash D_{P(V)}$ is a submersion.

We now determine the order of singularity of $\mathcal{K}$ near the diagonal $D_{P(V)}$. Let $[x]$, $\left[x^{\prime}\right]$ be points in $P(V)$ and let $\left(\eta_{k}, \eta_{k}^{\prime}\right)$ be a vector in $T_{[x]} P(V) \times T_{\left[x^{\prime}\right]} P(V)$ for each $1 \leq k \leq N-j$. First we have

$$
\begin{gathered}
\mathcal{K}_{\left([x],\left[x^{\prime}\right]\right)}\left(\left(\eta_{1}, \eta_{1}^{\prime}\right), \ldots,\left(\eta_{N-j}, \eta_{N-j}^{\prime}\right),\left(\bar{\eta}_{1}, \bar{\eta}_{1}^{\prime}\right), \ldots,\left(\bar{\eta}_{N-j}, \bar{\eta}_{N-j}^{\prime}\right)\right) \\
=\int_{\text {s`vect }\left(x, x^{\prime}\right)}\left(\left(\psi \circ p_{1}\right)^{*} \Omega^{d+q-1-j}\right)_{\left([x],\left[x^{\prime}\right], s\right)}\left(\left(\eta_{1}, \eta_{1}^{\prime}, \sigma_{1}\right), \ldots,\left(\eta_{N-j}, \eta_{N-j}^{\prime}, \sigma_{N-j}\right),\right. \\
\left.\left(\bar{\eta}_{1}, \bar{\eta}_{1}^{\prime}, \bar{\sigma}_{1}\right), \ldots,\left(\bar{\eta}_{N-j}, \bar{\eta}_{N-j}^{\prime}, \bar{\sigma}_{N-j}\right), \ldots\right) \\
=\int_{s \supset \operatorname{vect}\left(x, x^{\prime}\right)} \Omega_{s}^{d+q-1-j}\left(\sigma_{1}, \ldots, \sigma_{N-j}, \bar{\sigma}_{1}, \ldots, \bar{\sigma}_{N-j}, \ldots\right)
\end{gathered}
$$

where $\sigma_{k} \in T_{s} G(q, V)$ is such that $\left(\eta_{k}, \eta_{k}^{\prime}, \sigma_{k}\right) \in T_{\left([x],\left[x^{\prime}\right], s\right)} \Gamma^{\prime}$ and where the fiber

$$
q^{-1}\left([x],\left[x^{\prime}\right]\right)=\left\{s \supset \operatorname{vect}\left(x, x^{\prime}\right)\right\}
$$

is identified with $G\left(q-2, V /\left(\mathbb{C} x \oplus \mathbb{C} x^{\prime}\right)\right)$, which is of dimension $(q-2)(N+1-q)$, when $[x] \neq\left[x^{\prime}\right]$.

With $T_{[x]} P(V)=\operatorname{Hom}(\mathbb{C} x, V / \mathbb{C} x)$ and $T_{s} G(q, V)=\operatorname{Hom}(s, V / s)$, the condition $(\eta, \sigma) \in T_{([x], s)} \Gamma$ reduces to

$$
\sigma(x)=-\eta(x) \bmod s
$$

when $x \in s$. Thus we have $\sigma_{k}(x)=-\eta_{k}(x) \bmod s$ and $\sigma_{k}\left(x^{\prime}\right)=-\eta_{k}^{\prime}\left(x^{\prime}\right) \bmod s$ when vect $\left(x, x^{\prime}\right) \subset s$. Set

$$
y=\frac{x^{\prime}}{\left\|x^{\prime}\right\|}-\frac{\left\langle x^{\prime} \mid x\right\rangle x}{\left\|x^{\prime}\right\| \cdot\|x\|^{2}}
$$

in such a way that $\|y\|^{2}=\frac{\left\|x \wedge x^{\prime}\right\|^{2}}{\|x\|^{2}\left\|x^{\prime}\right\|^{2}}=1-\frac{\left|\left\langle x^{\prime} \mid x\right\rangle\right|^{2}}{\|x\|^{2}\left\|x^{\prime}\right\|^{2}}$. Then we take the lifting $\sigma_{k}$ defined by

$$
\|y\| \sigma_{k}=-\|y\| \frac{\langle. \mid x\rangle}{\|x\|^{2}} \otimes\left(\eta_{k}(x) \bmod s\right)+\frac{\langle\cdot \mid y\rangle}{\|y\|} \otimes\left\{\left(-\frac{\eta_{k}^{\prime}\left(x^{\prime}\right)}{\left\|x^{\prime}\right\|}+\frac{\left\langle x^{\prime} \mid x\right\rangle}{\left\|x^{\prime}\right\| \cdot\|x\|} \frac{\eta_{k}(x)}{\|x\|}\right) \bmod s\right\} .
$$

In particular $\|y\| \sigma_{k}$ is bounded when $\|y\|$ converges to 0 . In formula (19), by developping

$$
\sigma_{1} \wedge \ldots \wedge \sigma_{N-j} \wedge \bar{\sigma}_{1} \wedge \ldots \wedge \bar{\sigma}_{N-j}
$$

we choose in $\sigma_{k}$ the first term $-\frac{\langle. \mid x\rangle}{\|x\|^{2}} \otimes\left(\eta_{k}(x) \bmod s\right)$ or the second. But $\operatorname{dim}(V / s)=N+1-q$, so we choose at most $2(N+1-q)$ times the second. As a consequence, when $\|y\| \rightarrow 0$, we see that

$$
\|y\|^{2(N+1-q)} \mathcal{K}_{\left([x],\left[x^{\prime}\right]\right)}\left(\left(\eta_{1}, \eta_{1}^{\prime}\right), \ldots,\left(\eta_{N-j}, \eta_{N-j}^{\prime}\right),\left(\bar{\eta}_{1}, \bar{\eta}_{1}^{\prime}\right), \ldots,\left(\bar{\eta}_{N-j}, \bar{\eta}_{N-j}^{\prime}\right)\right)
$$

is bounded.

Theorem 2. There is a linear differential operator with smooth coefficients

$\mathcal{P}:\left\{\right.$ currents of bidegree $(q-j, q-j)$ on $\left.\mathbb{P}_{N}\right\} \rightarrow\left\{\right.$ currents of bidegree $(q, q)$ on $\left.\mathbb{P}_{N}\right\}$ of order $\leq 2 q-2$ such that id $=\mathcal{P C}^{*} \mathcal{C}$. 
Proof. Let $Q$ be a homogeneous polynomial function on $\mathbb{R}^{m}$ of degree $b$. With $\partial=\left(\frac{\partial}{\partial x_{1}}, \ldots, \frac{\partial}{\partial x_{m}}\right)$, let $Q(\partial)$ be the linear differential operator with constant coefficients associated to $Q$. If $u$ is a smooth function with compact support on $\mathbb{R}^{m}$, a solution $v$ of $u=Q(\partial) v$ is given by $v(x)=\int_{z \in \mathbb{R}^{m}} K(x, z) u(z) \mathrm{d} z$ with an integral kernel $K(x, z)=E(x-z)$ where the distribution $E$ satisfies

$$
Q(\partial)(E)=\delta \Leftrightarrow(\mathcal{F} E)(\xi)=\frac{1}{Q(2 \pi i \xi)} \Leftrightarrow E=\overline{\mathcal{F}}\left(\frac{1}{Q(2 \pi i x)}\right) .
$$

Then we have

$$
E(\xi)=\int_{\mathbb{R}^{m}} \frac{1}{Q(2 \pi i x)} e^{2 \pi i\langle x \mid \xi\rangle} \mathrm{d} x=\int_{\mathbb{R}^{m}} \frac{1}{Q\left(\frac{2 \pi i x}{\|\xi\|}\right)} e^{2 \pi i\left\langle x \mid \frac{\xi}{\|\xi\|}\right\rangle} \frac{\mathrm{d} x}{\|\xi\|^{m}}=\frac{1}{\|\xi\|^{m-b}} E\left(\frac{\xi}{\|\xi\|}\right)
$$

thus the singularity of $K$ is of order $m-b$ along the diagonal of $\mathbb{R}^{m} \times \mathbb{R}^{m}$. So we have here $2 N-b=2(N+1-$ $q)$.

\subsection{Approximation of closed currents of bidegree $(1,1)$ on $G(q, V)$ by divisors}

By using an inversion formula similar to that of Theorem 2, we prove now the following approximation property for closed $(1,1)$-currents on $G(q, V)=\mathbb{G}_{q-1, N}$ (see also [6]).

Proposition 6. Every closed $(1,1)$-current $\Theta$ on $G(q, V)$ can be written

$$
\Theta=\int_{H \in \operatorname{Div} G(q, V)} \lambda(H)[H]
$$

where $\lambda$ is a distribution on the space $\operatorname{Div} G(q, V)$ of divisors of $G(q, V)$ and $H$ is an effective divisor of $G(q, V)$. Moreover $\lambda$ can be calculated in the following way :

$$
\lambda(H)=\int_{s \in G(q, V)} \mathcal{M}(K(s, H)) \wedge \Theta(s)
$$

where $\mathcal{M}$ is a linear differential operator with smooth coefficients in $H$ and where the integral kernel $K$ is such that $K(., H)$ weakly converges to $K\left(., H_{0}\right)$ in $G(q, V)$ when $H$ converges to $H_{0}$.

Proof. Let $d_{G}=q(N+1-q)$ be the dimension of $G(q, V)$ and let

$$
\mathcal{R}: \mathcal{C}_{d_{G}-1, d_{G}-1}^{\infty}(G(q, V)) \rightarrow \mathcal{C}^{\infty}(\operatorname{Div} G(q, V))
$$

be the Radon transform defined by

$$
(\mathcal{R} \varphi)(H)=\int_{H} \varphi
$$

when $\varphi \in \mathcal{C}_{d_{G}-1, d_{G}-1}^{\infty}(G(q, V))$ and $H$ is an effective divisor of $G(q, V)$.

Then $\mathcal{R} \varphi=0$ if and only if $\varphi=\partial u+\bar{\partial} v$ with $u \in \mathcal{C}_{d_{G}-2, d_{G}-1}^{\infty}(G(q, V))$ and $v \in \mathcal{C}_{d_{G}-1, d_{G}-2}^{\infty}(G(q, V))$.

We identify a current of maximum bidegree on an irreducible component of $\operatorname{Div} G(q, V)$ with a distribution and we consider the transposed map

$$
{ }^{t} \mathcal{R}:\{\text { distributions on } \operatorname{Div} G(q, V)\} \rightarrow\{(1,1) \text {-currents on } G(q, V)\} .
$$

Its image is

$$
\operatorname{im}\left({ }^{t} \mathcal{R}\right)=(\operatorname{ker} \mathcal{R})^{\perp}=(\operatorname{im} \partial)^{\perp} \cap(\operatorname{im} \bar{\partial})^{\perp}=\operatorname{ker} \partial \cap \operatorname{ker} \bar{\partial} .
$$

In other words, since $\partial \Theta=0$ and $\bar{\partial} \Theta=0$, we can write $\Theta=\left({ }^{t} \mathcal{R}\right)(\lambda)$ for some distribution $\lambda$ on $\operatorname{Div} G(q, V)$.

Moreover, since ${ }^{t} \mathcal{R}$ is an integral transform, by [18] and by analogy with Theorem 2, we have an inversion formula

$$
\lambda=\left(\mathcal{M I}^{t} \mathcal{R}\right)(\lambda) \bmod \operatorname{ker}\left({ }^{t} \mathcal{R}\right)
$$


where $\mathcal{M}$ is a linear differential operator with smooth coefficients in $H$ and where $\mathcal{I}$ is an integral transform. Thus we can express

$$
\mathcal{I}(\Theta)(H)=\int_{s \in G(q, V)} K(s, H) \wedge \Theta(s)
$$

with an integral kernel $K$ satisfying : $K(., H)$ weakly converges to $K\left(., H_{0}\right)$ in $G(q, V)$ when $H$ converges to $H_{0}$.

As a conclusion, we can take

$$
\lambda(H)=(\mathcal{M I})(\Theta)(H)=\int_{s \in G(q, V)} \mathcal{M}(K(s, H)) \wedge \Theta(s) .
$$

\section{Chow transformation for $\mathbb{P}_{N}$ and parabolic subgroups of $S L(N+1, \mathbb{C})$}

With the point of view of homogeneous spaces (see $[13,19])$, we obtain again the equations (8) and (9) characterizing the closed $(1,1)$-currents on $G(q, V)$ which are in the image of the Chow transformation.

So we write

$$
\mathbb{P}_{N}=G L(N+1, \mathbb{C}) / G L(1, N, \mathbb{C})=S L(N+1, \mathbb{C}) /(G L(1, N, \mathbb{C}) \cap S L(N+1, \mathbb{C}))
$$

where $G L(1, N, \mathbb{C})=\left\{g \in G L(N+1, \mathbb{C})\right.$ such that $g\left(e_{0}\right)$ proportional to $\left.e_{0}\right\}$.

Also we have

$$
\mathbb{P}_{N}=U(N+1) /(U(N+1) \cap G L(1, N, \mathbb{C}))=U(N+1) / K
$$

with

$$
K=U(N+1) \cap G L(1, N, \mathbb{C})=U(1) \times U(N) .
$$

We write an element $M$ in $g l(N+1, \mathbb{C})=\mathcal{M}_{N+1, N+1}(\mathbb{C})$ in the following manner

$$
M=\left(\begin{array}{ll}
A & C \\
D & B
\end{array}\right)
$$

where $A \in \operatorname{gl}(1, \mathbb{C}), B \in \operatorname{gl}(N, \mathbb{C}), C \in n_{-}=\mathcal{M}_{1, N}(\mathbb{C})$ and $D \in n_{+}=\mathcal{M}_{N, 1}(\mathbb{C})$.

We denote by $a$ the complex vector space of the $\left(\begin{array}{ll}A & 0 \\ 0 & B\end{array}\right)$ in such a way that $g l(N+1, \mathbb{C})=a \oplus n_{-} \oplus n_{+}$.

The Lie algebra of $U(N+1)$ is

$$
\left\{M \in g l(N+1, \mathbb{C}), M^{*}=-M \text { i.e. } M \text { anti-Hermitian }\right\} .
$$

All $M \in g l(N+1, \mathbb{C})$ can be written in an unique way $M=M_{1}+i M_{2}$ where $M_{1}$ and $M_{2}$ are anti-Hermitian, thus $g l(N+1, \mathbb{C})$ is the complexified Lie algebra of the Lie algebra of $U(N+1)$.

Moreover $M$ given by (20) is anti-Hermitian when $A^{*}=-A, B^{*}=-B$ and $C=-D^{*}$. Thus the Lie algebra of $U(N+1)$ is $a_{0} \oplus t_{0}$ where $a_{0}$ is the real vector space of the $\left(\begin{array}{ll}A & 0 \\ 0 & B\end{array}\right)$ with $A, B$ anti-Hermitian and where $t_{0}$ is the real vector space of the $\left(\begin{array}{cc}0 & -D^{*} \\ D & 0\end{array}\right)$.

All $\left(\begin{array}{ll}0 & C \\ D & 0\end{array}\right)$ can be written in an unique way $\left(\begin{array}{cc}0 & -D_{1}^{*} \\ D_{1} & 0\end{array}\right)+i\left(\begin{array}{cc}0 & -D_{2}^{*} \\ D_{2} & 0\end{array}\right)$, thus we have $t_{0} \otimes_{\mathbb{R}} \mathbb{C}=n_{+} \oplus n_{-}$. Note that $a_{0}$ is the Lie algebra of $K$ and $a_{0} \otimes \mathbb{R} \mathbb{C}=a$. We set

$$
p: U(N+1) \rightarrow \mathbb{P}_{N}
$$


the canonical map, which is given by $p(g)=\left[g\left(e_{0}\right)\right]$. By differentiating at $I$ we obtain a $\mathbb{R}$-linear map

$$
a_{0} \oplus t_{0}=T_{I} U(N+1) \rightarrow T_{\left[e_{0}\right]}\left(\mathbb{P}_{N}\right)_{\mathbb{R}}
$$

given by

$$
M \rightarrow \frac{\mathrm{d}}{\mathrm{d} t}\left[e^{t M}\left(e_{0}\right)\right]_{\mid t=0}=\frac{\mathrm{d}}{\mathrm{d} t}\left[e_{0}+t M\left(e_{0}\right)\right]_{\mid t=0}=\mathrm{d} p_{I}(M)
$$

where we take $t \in \mathbb{R}$. By complexifying we deduce a $\mathbb{C}$-linear map

$$
g l(N+1, \mathbb{C})=T_{I} U(N+1) \otimes_{\mathbb{R}} \mathbb{C} \rightarrow T_{\left[e_{0}\right]}\left(\mathbb{P}_{N}\right)_{\mathbb{R}} \otimes_{\mathbb{R}} \mathbb{C}=T_{\left[e_{0}\right]} \mathbb{P}_{N} \oplus \overline{T_{\left[e_{0}\right]} \mathbb{P}_{N}}
$$

given by

$$
M \rightarrow\left(\frac{\mathrm{d}}{\mathrm{d} t}\left[e_{0}+t M\left(e_{0}\right)\right]_{\mid t=0}, \overline{\frac{\mathrm{d}}{\mathrm{d} t}\left[e_{0}-t M^{*}\left(e_{0}\right)\right]_{\mid t=0}}\right)
$$

where we take $t \in \mathbb{C}$. Its kernel is $a$ and it gives the identifications

$$
n_{+} \simeq T_{\left[e_{0}\right]} \mathbb{P}_{N} \text { and } n_{-} \simeq \overline{T_{\left[e_{0}\right]} \mathbb{P}_{N}} .
$$

For $g \in U(N+1)$, we set

$$
\lambda_{g}:(U(N+1), I) \rightarrow(U(N+1), g)
$$

the translation map given by $f \rightarrow g f$. The induced $\mathbb{C}$-linear map

$$
T_{I} U(N+1) \otimes_{\mathbb{R}} \mathbb{C} \rightarrow T_{g} U(N+1) \otimes_{\mathbb{R}} \mathbb{C} \rightarrow T_{\left[g\left(e_{0}\right)\right]} \mathbb{P}_{N} \oplus \overline{T_{\left[g\left(e_{0}\right)\right]} \mathbb{P}_{N}}
$$

gives the identifications

$$
n_{+} \simeq T_{\left[g\left(e_{0}\right)\right]} \mathbb{P}_{N} \text { and } n_{-} \simeq \overline{T_{\left[g\left(e_{0}\right)\right]} \mathbb{P}_{N}} .
$$

Let $u$ be a smooth differential $(q-1, q-1)$-form on $\mathbb{P}_{N}$. Therefore, from

$$
u_{\left[g\left(e_{0}\right)\right]} \in \bigwedge^{q-1} T_{\left[g\left(e_{0}\right)\right]}^{*} \mathbb{P}_{N} \otimes \bigwedge^{q-1} \overline{T_{\left[g\left(e_{0}\right)\right]}^{*} \mathbb{P}_{N}}
$$

we deduce an element $\left(\left(p \circ \lambda_{g}\right)^{*} u\right)(I)=\tilde{u}(g) \in \wedge^{q-1} n_{+}^{*} \otimes \wedge^{q-1} n_{-}^{*}$.

For $k \in K$ and $M \in g l(N+1, \mathbb{C})$, we set

$$
\operatorname{ad}(k)(M)=\frac{\mathrm{d}}{\mathrm{d} t}\left(k e^{t M} k^{-1}\right)_{\mid t=0}=k M k^{-1}
$$

which satisfies $a d(k)\left(n_{+}\right)=n_{+}$and $a d(k)\left(n_{-}\right)=n_{-}$. Since $\left[k\left(e_{0}\right)\right]=\left[e_{0}\right]$, we have $p \circ \lambda_{g k}=p \circ \lambda_{g} \circ a d(k)$ and we can evaluate

$$
\tilde{u}(g k)=\left(\operatorname{ad}(k)^{*}\left(p \circ \lambda_{g}\right)^{*} u\right)(I)=\operatorname{ad}(k)^{*}\left\{\left(\left(p \circ \lambda_{g}\right)^{*} u\right)(I)\right\}=a d(k)^{*} \tilde{u}(g) .
$$

Proposition 7. A smooth differential ( $q-1, q-1)$-form u on $\mathbb{P}_{N}$ corresponds to a smooth function ũ on $U(N+1)$ with values in $\wedge^{q-1} n_{+}^{*} \otimes \wedge^{q-1} n_{-}^{*}$, which satisfies the property (22) of right invariance by $K$.

For $h \in U(N+1)$, let us remark that

$$
\widetilde{h^{*} u}(g)=\tilde{u}(h g)
$$

because $\left(\left(p \circ \lambda_{g}\right)^{*} h^{*} u\right)(I)=\left(\left(p \circ \lambda_{h g}\right)^{*} u\right)(I)$.

We now express in terms of $\tilde{u}$ the Chow transform $\mathcal{C}(u)$ of $u$, which is a function on $\mathbb{G}_{q-1, N}=G(q, V)$.

Every $s \in G(q, V)$ can be written $s=\operatorname{vect}\left(g\left(e_{0}\right), \ldots, g\left(e_{q-1}\right)\right)=g\left(\mathbb{C}^{q}\right)$ for some $g \in U(N+1)$ and $\operatorname{vect}\left(g^{\prime}\left(e_{0}\right), \ldots, g^{\prime}\left(e_{q-1}\right)\right)=\operatorname{vect}\left(g\left(e_{0}\right), \ldots, g\left(e_{q-1}\right)\right)$ if and only if

$$
g^{-1} g^{\prime} \in U\left(\operatorname{vect}\left(e_{0}, \ldots, e_{q-1}\right)\right) \times U\left(\operatorname{vect}\left(e_{0}, \ldots, e_{q-1}\right)^{\perp}\right)=U(q) \times U(N+1-q) .
$$

Therefore we have

$$
\mathbb{G}_{q-1, N}=U(N+1) /(U(q) \times U(N+1-q)) .
$$


But $\mathcal{C}(u)$ is obtained by integration of $u$ on the projective subspaces of $\mathbb{P}_{N}$ of dimension $q-1$ i.e.

$$
\mathcal{C}(u)\left(\operatorname{vect}\left(h\left(e_{0}\right), \ldots, h\left(e_{q-1}\right)\right)\right)=\int_{P\left(\operatorname{vect}\left(h\left(e_{0}\right), \ldots, h\left(e_{q-1}\right)\right)\right)} u=\int_{\mathbb{P}_{q-1}} h^{*} u
$$

with $P\left(\operatorname{vect}\left(e_{0}, \ldots, e_{q-1}\right)\right)=P\left(\mathbb{C}^{q}\right)$ equal to

$$
\mathbb{P}_{q-1}=(U(q) \times U(N+1-q)) /(U(1) \times U(q-1) \times U(N+1-q)) .
$$

Let $F \in \mathcal{C}^{\infty}\left(\mathbb{P}_{q-1}\right)$ be such that $\left(h^{*} u\right)_{\mid \mathbb{P}_{q-1}}=F \omega_{\mid \mathbb{P}_{q-1}}^{q-1}$ with $\omega$ the Fubini-Study form on $\mathbb{P}_{N}=P(V)$. A point in $\mathbb{P}_{q-1}$ can be written $\left[g\left(e_{0}\right)\right]$ for some $g \in U(q) \times U(N+1-q)$ and

$$
T_{\left[g\left(e_{0}\right)\right]} \mathbb{P}_{q-1}=\operatorname{Hom}\left(\mathbb{C g}\left(e_{0}\right), \mathbb{C}^{q} / \mathbb{C g}\left(e_{0}\right)\right) .
$$

Since $g\left(e_{0}\right), \ldots, g\left(e_{q-1}\right)$ is an orthonormal basis of $\mathbb{C}^{q}$, an orthonormal basis of $T_{\left[g\left(e_{0}\right)\right]} \mathbb{P}_{q-1}$ is given by the

$$
w_{k}=g\left(e_{0}\right)^{-1} \otimes\left(g\left(e_{k}\right) \bmod \mathbb{C g}\left(e_{0}\right)\right)=\frac{\mathrm{d}}{\mathrm{d} t}\left[g\left(e_{0}\right)+\operatorname{tg}\left(e_{k}\right)\right]_{\mid t=0}=\frac{\mathrm{d}}{\mathrm{d} t}\left[g\left(I+t M_{k}\right)\left(e_{0}\right)\right]_{\mid t=0}
$$

for $1 \leq k \leq q-1$ and with $M_{k}=e_{0}^{*} \otimes e_{k} \in n_{+}$and $M_{k}^{*}=e_{k}^{*} \otimes e_{0} \in n_{-}$. Thus

$$
\begin{aligned}
\widetilde{h^{*} u}(g)\left(M_{1}, \ldots, M_{q-1},-M_{1}^{*}, \ldots,-M_{q-1}^{*}\right) & =\left(h^{*} u\right)_{\left[g\left(e_{0}\right)\right]}\left(w_{1}, \ldots, w_{q-1}, \bar{w}_{1}, \ldots, \bar{w}_{q-1}\right) \\
& =F\left(\left[g\left(e_{0}\right)\right]\right) \omega_{\left[g\left(e_{0}\right)\right]}^{q-1}\left(w_{1}, \ldots, w_{q-1}, \bar{w}_{1}, \ldots, \bar{w}_{q-1}\right)
\end{aligned}
$$

which is equal to

$$
\begin{aligned}
& F\left(\left[g\left(e_{0}\right)\right]\right) \omega_{\left[e_{0}\right]}^{q-1}\left(\frac{\mathrm{d}}{\mathrm{d} t}\left[e_{0}+t e_{1}\right]_{\mid t=0}, \ldots, \frac{\mathrm{d}}{\mathrm{d} t}\left[e_{0}+t e_{q-1}\right]_{\mid t=0}, \overline{\frac{\mathrm{d}}{\mathrm{d} t}\left[e_{0}+t e_{1}\right]_{\mid t=0}}, \ldots, \overline{\frac{\mathrm{d}}{\mathrm{d} t}\left[e_{0}+t e_{q-1}\right]_{\mid t=0}}\right) \\
= & \frac{i^{(q-1)^{2}}}{(2 \pi)^{q-1}}(q-1) ! F\left(\left[g\left(e_{0}\right)\right]\right) .
\end{aligned}
$$

Lemma 4. The smooth function $F$ on $\mathbb{P}_{q-1}$ such that $\left(h^{*} u\right)_{\mathbb{P}_{q-1}}=F \omega_{\mid \mathbb{P}_{q-1}}^{q-1}$ is given by

$$
F\left(\left[g\left(e_{0}\right)\right]\right)=C^{-1} \tilde{u}(h g)\left(M_{1}, \ldots, M_{q-1}, M_{1}^{*}, \ldots, M_{q-1}^{*}\right)
$$

with $C=\frac{i^{q^{2}-1}}{(2 \pi)^{q-1}}(q-1)$ ! for every $g \in U(q) \times U(N+1-q)$.

As a consequence, if we write $s=h\left(\mathbb{C}^{q}\right)$ with $h \in U(N+1)$, we have

$$
\begin{aligned}
\mathcal{C}(u)(s) & =\int_{g \in U(q) \times U(N+1-q)} F\left(\left[g\left(e_{0}\right)\right]\right) \mathrm{d} \mu(g) \\
& =C^{-1} \int_{g \in U(q) \times U(N+1-q)} \tilde{u}(h g)\left(M_{1}, \ldots, M_{q-1}, M_{1}^{*}, \ldots, M_{q-1}^{*}\right) \mathrm{d} \mu(g)
\end{aligned}
$$

where $\mu$ is the left invariant measure on $U(q) \times U(N+1-q)$ with total mass equal to 1 .

Note that we also have

$$
\mathbb{G}_{q-1, N}=G L(N+1, \mathbb{C}) / G L(q, N+1-q, \mathbb{C})
$$

where $G L(q, N+1-q, \mathbb{C})=\left\{g \in G L(N+1, \mathbb{C})\right.$ such that $\left.g\left(\operatorname{vect}\left(e_{0}, \ldots, e_{q-1}\right)\right)=\operatorname{vect}\left(e_{0}, \ldots, e_{q-1}\right)\right\}$.

Moreover $p(g)=\left[g\left(e_{0}\right)\right]$ is still defined as a holomorphic map

$$
p: G L(N+1, \mathbb{C}) \rightarrow \mathbb{P}_{N}
$$

with $\mathrm{d} p_{g}$ the differential at $g$ obtained from

$$
M \rightarrow \frac{\mathrm{d}}{\mathrm{d} t}\left[g(I+t M)\left(e_{0}\right)\right]_{\mid t=0}
$$

and which still gives the identification $n_{+} \simeq T_{\left[g\left(e_{0}\right)\right]} \mathbb{P}_{N}$. Therefore the vectorial function $\tilde{u}(g)$ is still defined for $g \in G L(N+1, \mathbb{C})$. 
Lemma 5. For $s=h\left(\mathbb{C}^{q}\right)$ with $h \in G L(N+1, \mathbb{C})$, we have

$$
\mathcal{C}(u)(s)=C^{-1} \int_{g \in U(q) \times U(N+1-q)} \tilde{u}(h g)\left(M_{1}, \ldots, M_{q-1}, M_{1}^{*}, \ldots, M_{q-1}^{*}\right) \mathrm{d} \mu(g) .
$$

By writing $s=\operatorname{vect}\left(h\left(e_{0}\right), \ldots, h\left(e_{q-1}\right)\right)$, we denote by $\left(h_{i k}\right)_{0 \leq i \leq N}$ the coordinates of $h\left(e_{k}\right)$ with respect to the basis $e_{0}, \ldots, e_{N}$. For $I=\left(i_{0}, \ldots, i_{q-1}\right)$ with $0 \leq i_{0}<\ldots<i_{q-1} \leq N$, we consider the differential operator

$$
\operatorname{det}\left(\frac{\partial}{\partial h_{i k}}\right) \underset{\substack{i \in I \\ 0 \leq k \leq q-1}}{ }=\sum_{\sigma} \varepsilon(\sigma) \frac{\partial^{q}}{\partial h_{i_{\sigma_{0}}, 0} \ldots \partial h_{i_{\sigma_{q-1}}, q-1}}
$$

where $\sigma$ is each permutation of $\{0, \ldots, q-1\}$. We evaluate on the previous function $\mathcal{C}(u)$ of $h$ the differential operator

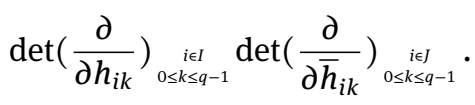

To this end we use that

$$
\frac{\partial^{q}}{\partial h_{i_{\sigma_{0}}, 0} \ldots \partial h_{i_{\sigma_{q-1}}, q-1}}(\tilde{u}(h g))=\sum_{0 \leq m_{0}, \ldots, m_{q-1} \leq N} \frac{\partial^{q} \tilde{u}}{\partial h_{i_{0}, m_{0}}^{\prime} \ldots \partial h_{i_{\sigma_{q-1}}, m_{q-1}}}(h g) g_{0, m_{0}} \ldots g_{q-1, m_{q-1}}
$$

which gives

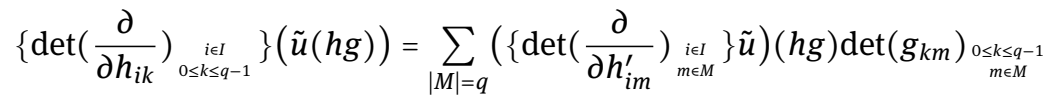

with $M=\left(m_{0}, \ldots, m_{q-1}\right)$ where $0 \leq m_{0}<\ldots<m_{q-1} \leq N$. Since $g \in U(q) \times U(N+1-q)$ we can take $0 \leq m_{k} \leq q-1$ thus necessarily $m_{k}=k$. So we conclude that

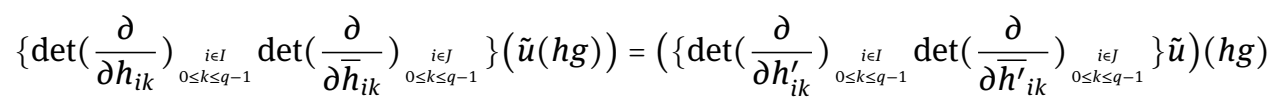

since

$$
\mid \operatorname{det}\left(g_{k m}\right) \substack{0 \leq k \leq q-1 \\ 0 \leq m \leq q-1}^{2}=1 .
$$

Lemma 6. The smooth function on $U(N+1)$

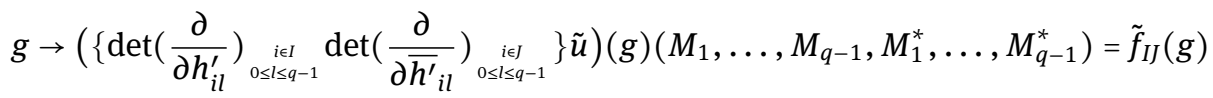

is invariant by the right action of $K=U(1) \times U(N)$ and thus induces a smooth function $f_{I J}$ on $\mathbb{P}_{N}$.

Proof. For all $g \in G L(N+1, \mathbb{C})$ and all $k \in K$, we rewrite formula (22) i.e.

$$
\tilde{u}(g k)=a d(k)^{*} \tilde{u}(g) .
$$

By applying the following differential operator

$$
\left.\operatorname{det}\left(\frac{\partial}{\partial g_{i l}}\right) \underset{\substack{i \in I \\ 0 \leq \leq \leq q-1}}{\operatorname{det}\left(\frac{\partial}{\partial \bar{g}_{i l}}\right)}\right)_{\substack{i \in I \\ 0 \leq \leq \leq q-1}}
$$

to both members, we obtain for all $k \in K$ the relation

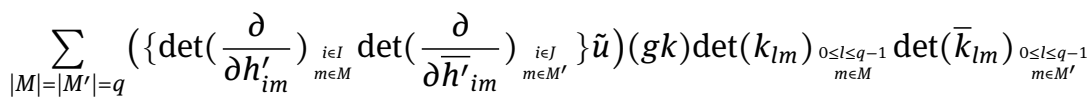

$$
\begin{aligned}
& \left.=\operatorname{ad}(k)^{*}\left(\left\{\operatorname{det}\left(\frac{\partial}{\partial h_{i l}^{\prime}}\right) \underset{\substack{i \in I \\
0 \leq \leq \leq q-1}}{\operatorname{det}\left(\frac{\partial}{\partial \bar{h}^{\prime}}\right)}\right) \underset{\substack{i l \\
0 \leq \leq \leq q-1}}{0}\right\} \tilde{u}\right)(g) .
\end{aligned}
$$

By evaluating on $M_{1} \wedge \ldots \wedge M_{q-1} \wedge M_{1}^{*} \wedge \ldots \wedge M_{q-1}^{*}$ and by identifying, it comes

$$
\left.\left(\left\{\operatorname{det}\left(\frac{\partial}{\partial h_{i l}^{\prime}}\right) \underset{\substack{i \in I \\ 0 \leq \leq \leq q-1}}{\operatorname{det}\left(\frac{\partial}{\partial \bar{h}^{\prime}}\right)}\right)_{\substack{i \in J \\ 0 \leq \leq \leq \leq-1}}\right\} \tilde{u}\right)(g k)\left(M_{1}, \ldots, M_{q-1}, M_{1}^{*}, \ldots, M_{q-1}^{*}\right) \mid \operatorname{det}\left(k_{l m}\right)_{\substack{0 \leq \leq \leq q-1 \\ 0 \leq m \leq q-1}}^{2}
$$




$$
\left.=\left(\left\{\operatorname{det}\left(\frac{\partial}{\partial h_{i l}^{\prime}}\right) \underset{\substack{i \in I \\ 0 \leq \leqslant \leq q-1}}{\operatorname{det}\left(\frac{\partial}{\partial \bar{h}^{\prime} i l}\right)}\right)_{\substack{i \in I \\ 0 \leq \leq \leq q-1}}\right\} \tilde{u}\right)(g)\left(M_{1}, \ldots, M_{q-1}, M_{1}^{*}, \ldots, M_{q-1}^{*}\right)\left|\operatorname{det}\left(k_{l m}\right)_{\substack{1 \leq l \leq q-1 \\ 1 \leq m \leq q-1}}\right|^{2}
$$

thanks to the development

$$
\left(e_{0}^{*} \otimes k\left(e_{1}\right)\right) \wedge \ldots \wedge\left(e_{0}^{*} \otimes k\left(e_{q-1}\right)\right)=\sum_{0 \leq m_{1}, \ldots, m_{q-1} \leq N} k_{m_{1}, 1} \ldots k_{m_{q-1}, q-1}\left(e_{0}^{*} \otimes e_{m_{1}}\right) \wedge \ldots \wedge\left(e_{0}^{*} \otimes e_{m_{q-1}}\right) .
$$

As a conclusion, for $h \in G L(N+1, \mathbb{C})$ we obtain

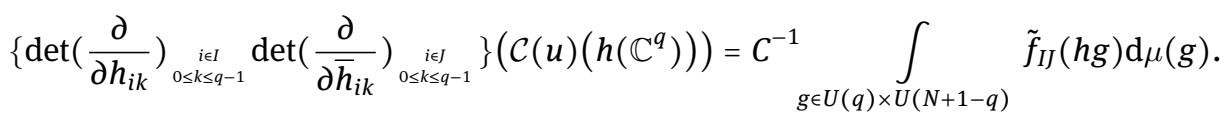

On the over hand, the projective $(q-1)$-dimensional Radon transform of $f_{I J}$ is given by

$$
\mathcal{R}_{q-1}\left(f_{I J}\right)(s)=\int_{P(s)} f_{I J} \omega^{q-1}=\int_{\mathbb{P}_{q-1}}\left(h^{*} f_{I J}\right) \omega^{q-1}=\int_{g \in U(q) \times U(N+1-q)} \tilde{f}_{I J}(h g) \mathrm{d} \mu(g)
$$

by writing $s=h\left(\mathbb{C}^{q}\right)$ with $h \in U(N+1)$ and by using (23).

To retrieve equations (8) and (9), it remains to evaluate on $\mathcal{R}_{q-1}\left(f_{I J}\right)$ the differential operator

$$
\frac{\partial^{2}}{\partial h_{i k} \partial h_{i^{\prime} k^{\prime}}}-\frac{\partial^{2}}{\partial h_{i^{\prime} k} \partial h_{i k^{\prime}}}
$$

with $0 \leq k, k^{\prime} \leq q-1$ and $0 \leq i, i^{\prime} \leq N$. To this end we use that

$$
\frac{\partial^{2}}{\partial h_{i k} \partial h_{i^{\prime} k^{\prime}}}\left(\tilde{f}_{I J}(h g)\right)=\sum_{0 \leq m, m^{\prime} \leq N}\left(\frac{\partial^{2} \tilde{f}_{I J}}{\partial h_{i m}^{\prime} \partial h_{i^{\prime} m^{\prime}}^{\prime}}\right)(h g) g_{k m} g_{k^{\prime} m^{\prime}}
$$

with by definition

$$
\left(\frac{\partial^{2} \tilde{f}_{I J}}{\partial h_{i m}^{\prime} \partial h_{i^{\prime} m^{\prime}}^{\prime}}\right)(g)=\frac{\partial^{2}}{\partial t \partial s}\left(\tilde{f}_{I J}\left(g+t e_{m}^{*} \otimes e_{i}+s e_{m^{\prime}}^{*} \otimes e_{i^{\prime}}\right)\right)_{\mid(t, s)=(0,0)} .
$$

But by Lemma 6 we can express

$$
\tilde{f}_{I J}\left(g+t e_{m}^{*} \otimes e_{i}+s e_{m^{\prime}}^{*} \otimes e_{i^{\prime}}\right)=f_{I J}\left(\left[g\left(e_{0}\right)+t \delta_{m, 0} e_{i}+s \delta_{m^{\prime}, 0} e_{i^{\prime}}\right]\right) .
$$

Necessarily $m=0=m^{\prime}$, so it is equal to $f_{I J}\left(\left[g\left(e_{0}\right)+t e_{i}+s e_{i^{\prime}}\right]\right)$ and the derivative of order 2 in $(0,0)$ is invariant by permuting $i$ and $i^{\prime}$.

Theorem 3. The Chow transform $U=\mathcal{C}(u)$ of a smooth differential $(q-1, q-1)$-form $u$ on $\mathbb{P}_{N}$ is a smooth function on $\mathbb{G}_{q-1, N}$. The smooth function $\tilde{U}(h)=U\left(h\left(\mathbb{C}^{q}\right)\right)$ for $h \in G L(N+1, \mathbb{C})$ satisfies the linear differential equations

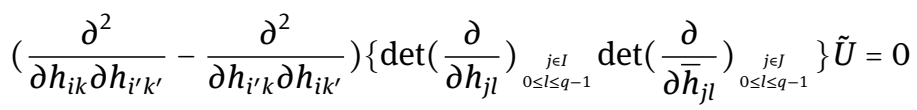

and

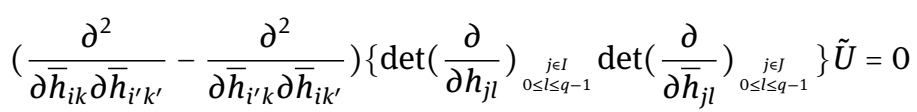

for all $|I|=|J|=q$, all $0 \leq k, k^{\prime} \leq q-1$ and all $0 \leq i, i^{\prime} \leq N$. 


\section{Chow transformation and geometry of the cotangent bundle}

\subsection{Chow transform of the conormal of a current}

Here we use the geometry of the cotangent bundle (see $[11,17])$ to study the injectivity of the Chow transformation.

For $([x], s) \in \Gamma$, the fiber of the conormal to $\Gamma$ in $P(V) \times G(q, V)$ at $([x], s)$ is

$$
N_{([x], s)}^{*} \Gamma=\left(\left(T_{[x]} P(V) \times T_{s} G(q, V)\right) / T_{([x], s)} \Gamma\right)^{*}
$$

i.e. the space of linear forms on $T_{[x]} P(V) \times T_{s} G(q, V)$ which are equal to 0 on $T_{([x], s)} \Gamma$. Therefore

$$
N^{*} \Gamma \hookrightarrow T^{*}(P(V) \times G(q, V))_{\mid \Gamma} \rightarrow T^{*} P(V) \times T^{*} G(q, V) .
$$

We still denote by $\pi: V \backslash\{0\} \rightarrow P(V)$ the natural map.

Lemma 7. Let $(\xi, u) \in T_{[x]}^{*} P(V) \times T_{s}^{*} G(q, V)$ be such that $\xi \neq 0$ and $u \neq 0$. There is $c \in \mathbb{C}^{*}$ such that $(\xi, c u) \in$ $N_{([x], s)}^{*} \Gamma$ if and only if

(i) $x \in S$,

(ii) $\mathrm{s} \subset \mathrm{d} \pi_{x}^{-1}(\operatorname{ker} \xi) \stackrel{\text { def }}{=} H \subset V$,

(iii) $\operatorname{ker} u=\{\sigma \in \operatorname{Hom}(s, V / s), \sigma(x) \in H / s\}$.

Proof. Let $E$ be the holomorphic vector bundle on $P(V) \times G(q, V)$ defined by

$$
E_{([x], s)}=\operatorname{Hom}(\mathbb{C} x, V / s)
$$

and let $h$ be the holomorphic section of $E$ equal at $([x], s)$ to the natural morphism $\mathbb{C} x \rightarrow V / s$. The set of zeroes of $h$ is $\Gamma$. With $\left(e_{0}, \ldots, e_{N-q}\right)$ a holomorphic local frame of $E$, we can write $h=\sum_{i} h_{i} e_{i}$. At a point $([x], s)$ of $\Gamma$, then $\sum_{i} \mathrm{~d} h_{i} \otimes e_{i}$ is a surjective morphism

$$
T_{([x], s)}(P(V) \times G(q, V)) \rightarrow E_{([x], s)}
$$

independent of the choice of the holomorphic local frame. The kernel of this morphism is $T_{([x], s)} \Gamma$, so the normal bundle to $\Gamma$ is isomorphic to $E_{\mid \Gamma}$ and

$$
N_{([x], s)}^{*} \Gamma=\operatorname{Hom}(V / s, \mathbb{C} x) .
$$

We consider $\xi \in \operatorname{Hom}(V / \mathbb{C} x, \mathbb{C} x)$ and $u \in \operatorname{Hom}(V / s, s)$. There is $c \in \mathbb{C}^{*}$ such that $(\xi, c u) \in N_{([x], s)}^{*} \Gamma$ if and only if there is $\zeta \in \operatorname{Hom}(V / s, \mathbb{C} x)$ satisfying

$$
\xi=\zeta \circ p \text { and } u \text { proportional to } i \circ \zeta
$$

with $p: V / \mathbb{C} x \rightarrow V / s$ and $i: \mathbb{C} x \rightarrow s$ the natural maps.

The existence of $\zeta$ such that $\xi=\zeta \circ p$ is equivalent to ker $p \subset \operatorname{ker} \xi$ i.e. $s \subset H$ and then we have $\zeta(y \bmod s)=$ $\xi(y \bmod \mathbb{C} x)$ for all $y \in V$. By writing $\zeta=x \otimes \lambda$ with $\lambda \in(V / s)^{*}$, we have for $\sigma \in \operatorname{Hom}(s, V / s)$ the equality $\langle i \circ \zeta, \sigma\rangle=\lambda(\sigma(x))$. In such a way, the hyperplane orthogonal to $i \circ \zeta$ is

$$
\text { ker } u=\{\sigma \in \operatorname{Hom}(s, V / s), \sigma(x) \in \operatorname{ker} \lambda\}=\{\sigma \in \operatorname{Hom}(s, V / s), \sigma(x) \in H / s\} .
$$

We denote by $\Phi$ respectively $\Psi$ the restriction to the conormal $N^{*} \Gamma$ of the canonical projection $T^{*} P(V) \times$ $T^{*} G(q, V) \rightarrow T^{*} P(V)$ respectively $T^{*} P(V) \times T^{*} G(q, V) \rightarrow T^{*} G(q, V)$.

Let $Z \subset P(V)$ be an irreducible algebraic subset of codimension $q$. Then

$$
N^{*} Z=\left(T P(V)_{\mid Z} / T Z\right)^{*} \hookrightarrow T^{*} P(V)_{\mid Z} \hookrightarrow T^{*} P(V) .
$$

We use the double fibration

to transform $N^{\star} Z$.

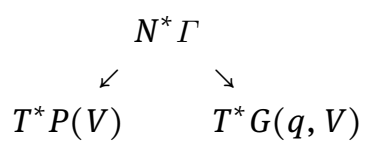


Proposition 8. For $Z \subset P(V)$ algebraic with pure codimension $q$, let $\Sigma=\mathcal{C}(Z)$ be the Chow divisor of $Z$. Then we have $\Psi_{\star} \Phi^{*}\left(N^{*} Z\right)=N^{*} \Sigma$ and conversely $\Phi_{*} \Psi^{*}\left(N^{*} \Sigma\right)=N^{*} Z$.

Proof. For the first equality, let $u \in T_{s}^{*} G(q, V)$ be such that $u \in \Psi_{*} \Phi^{*}\left(N^{\star} Z\right)$. There is $\xi \in N^{*} Z$ such that $(\xi, u) \in N^{*} \Gamma$, then $\xi \in T_{[x]}^{*} P(V)$ with $[x] \in Z$. By Lemma 7, $x$ belongs to $s$ so $P(s)$ cuts $Z$ and then $s \in \Sigma$. Since $\xi$ is equal to 0 on $T_{[x]} Z+T_{[x]} P(s)$ which is a hyperplane in $T_{[x]} P(V)$, we have $\operatorname{ker} \xi=T_{[x]} Z+T_{[x]} P(s)$ i.e. $H=\mathrm{d} \pi_{x}^{-1}(\operatorname{ker} \xi)=s+\mathrm{d} \pi_{x}^{-1}\left(T_{[x]} Z\right)$. By [25] we can assume $Z \cap P(s)=\{[x]\}$ and then

$$
T_{s} \Sigma=\left\{\sigma \in \operatorname{Hom}(s, V / s), \sigma(x) \in\left(s+T_{\chi} \pi^{-1}(Z)\right) / s\right\} .
$$

In such a way, by Lemma 7, we obtain ker $u=T_{s} \Sigma$, thus $u \in N^{*} \Sigma$.

For the second equality, let $\xi \in T_{[x]}^{*} P(V)$ be such that $\xi \in \Phi_{*} \Psi^{*}\left(N^{*} \Sigma\right)$. There is $u \in N^{*} \Sigma$ such that $(\xi, u) \in N^{*} \Gamma$. Then $u \in T_{s}^{*} G(q, V)$ with some $s \in \Sigma$. With $\{[x]\}=Z \cap P(s)$, we apply (26) to obtain

$$
\text { ker } u=\left\{\sigma \in \operatorname{Hom}(s, V / s), \sigma(x) \in\left(s+T_{x} \pi^{-1}(Z)\right) / s\right\} \text {. }
$$

Then by Lemma 7, we have ker $\xi \supset T_{[x]} Z$, thus $\xi \in N^{*} Z$.

We now extend the formula

$$
N^{*} Z=\Phi_{*} \Psi^{*} \Psi_{*} \Phi^{*}\left(N^{*} Z\right)
$$

resulting of Proposition 8, by replacing $Z$ by a closed positive current $T$ of bidegree $(q, q)$ on $P(V)$ and by writing as in Proposition 3

$$
T=\lim _{\nu} Z_{\nu} \text { and } T^{*}=\lim _{\nu} Z_{\nu}^{*}
$$

with $Z_{\nu}$ effective algebraic cycle of codimension $q$ in $P(V)$ and $Z_{\nu}^{*}$ the dual cycle of $Z_{\nu}$ in $P\left(V^{*}\right)$.

First we recall that there is a surjective morphism

$$
\alpha: T^{*} P(V) \backslash 0_{P(V)} \rightarrow\left\{([x],[\lambda]) \in P(V) \times P\left(V^{*}\right) \text { such that } \lambda(x)=0\right\}
$$

which associates $\alpha(\xi)=([x],[\lambda])$ to $\xi \in T_{[x]}^{*} P(V)=\operatorname{Hom}(V / \mathbb{C} x, \mathbb{C} x)$ equal to $\lambda \otimes x$ with $\lambda(x)=0$.

Then $\xi \in N_{[x]}^{*} Z$ if and only if ker $\lambda \supset \mathrm{d} \pi_{x}^{-1}\left(T_{[x]} Z\right)$ i.e. $\alpha(\xi)$ belongs to $W_{Z}$ with

$$
W_{Z}=\left\{([x],[\lambda]) \in P(V) \times P\left(V^{*}\right) \text { such that }[x] \in Z \text { and } P(\operatorname{ker} \lambda) \supset \mathbb{T}_{[x]} Z\right\}
$$

where $\mathbb{T}_{[x]} Z=P\left(\mathrm{~d} \pi_{x}^{-1}\left(T_{[x]} Z\right)\right) \subset P(V)$.

In other words $\left(N^{*} Z\right) \backslash 0_{Z}=\alpha^{-1}\left(W_{Z}\right)$ and $W_{Z}$ is an algebraic subvariety of $P(V) \times P\left(V^{*}\right)$ with $\operatorname{dim} W_{Z}=$ $N-1$. The image by $\operatorname{pr}_{2}$ of $W_{Z}$ is $\operatorname{pr}_{2}\left(W_{Z}\right)=Z^{*} \subset P\left(V^{*}\right)$, for which we expect that

$$
\operatorname{codim} Z^{*}=N+1-q \Leftrightarrow \operatorname{dim} Z^{*}=q-1=\operatorname{codim} Z-1
$$

by Proposition 3. We equip $P\left(V^{*}\right)$ with the dual metric $\omega^{*}$ and $P(V) \times P\left(V^{*}\right)$ with the induced metric.

Proposition 9. In the sense of currents, we have the following equalities

(i) $\left[N^{*} Z\right]=\alpha^{*}\left[W_{Z}\right]$,

(ii) $\left(\begin{array}{c}N-1 \\ q-1\end{array}\right) \operatorname{pr}_{2 *}\left(\frac{\left[W_{Z}\right]}{\operatorname{deg} W_{Z}} \wedge \operatorname{pr}_{1}^{*} \omega^{N-q}\right)=\frac{\left[Z^{*}\right]}{\operatorname{deg} Z^{*}}$ where $\operatorname{deg} W_{Z}=\left(\begin{array}{c}N-1 \\ q-1\end{array}\right) \operatorname{deg} Z$ is the degree of $W_{Z}$.

Proof. With $\beta$ the restriction of $\mathrm{pr}_{2}$ to $W_{Z}$, the following diagram

$$
\begin{array}{ccc}
W_{Z} \stackrel{i_{0}}{\hookrightarrow} & P(V) \times P\left(V^{*}\right) \\
\beta & \downarrow & \downarrow^{\mathrm{pr}_{2}} \\
Z^{*} \stackrel{j}{\hookrightarrow} & P\left(V^{*}\right)
\end{array}
$$

is commutative, where $i_{0}$ and $j$ are the natural injections. With these notations, we have

$$
\operatorname{pr}_{2 *}\left(\left[W_{Z}\right] \wedge \operatorname{pr}_{1}^{*} \omega^{N-q}\right)=\operatorname{pr}_{2 *} i_{0 *} i_{0}^{*} \operatorname{pr}_{1}^{*} \omega^{N-q}=j_{*} \beta_{*}\left(\left(\operatorname{pr}_{1}^{*} \omega^{N-q}\right){ }_{\mid W_{Z}}\right) .
$$


The fibers of $\beta$ are of dimension $N-q=\operatorname{dim} W_{Z}-\operatorname{dim} Z^{*}$ and the function

$$
\beta_{*}\left(\left(\operatorname{pr}_{1}^{*} \omega^{N-q}\right)_{\mid W_{Z}}\right)([\lambda])=\int_{\beta^{-1}([\lambda])} \omega^{N-q}
$$

is continuous with respect to $[\lambda] \in Z^{*}$. Since it is a positive integer, when $Z$ is irreducible, this function is a constant $M$. We obtain

$$
\operatorname{pr}_{2 *}\left(\left[W_{Z}\right] \wedge \operatorname{pr}_{1}^{*} \omega^{N-q}\right)=M\left[Z^{*}\right] .
$$

Then by testing on $\left(\omega^{*}\right)^{q-1}$ it comes

$$
M \operatorname{deg} Z^{*}=M \int_{Z^{*}}\left(\omega^{*}\right)^{q-1}=\int_{W_{Z}} \operatorname{pr}_{1}^{*} \omega^{N-q} \wedge \operatorname{pr}_{2}^{*}\left(\omega^{*}\right)^{q-1}
$$

and it remains to evaluate deg $W_{Z}$. By the binomial formula, for $0 \leq k \leq N-1$, we have to calculate

$$
\int_{W_{Z}} \operatorname{pr}_{1}^{*} \omega^{N-1-k} \wedge \operatorname{pr}_{2}^{*}\left(\omega^{*}\right)^{k}=\int_{P\left(V^{*}\right)} \operatorname{pr}_{2 *}\left(\left[W_{Z}\right] \wedge \operatorname{pr}_{1}^{*} \omega^{N-1-k}\right) \wedge\left(\omega^{*}\right)^{k} .
$$

But in a similar manner

$$
\operatorname{pr}_{2 *}\left(\left[W_{Z}\right] \wedge \operatorname{pr}_{1}^{*} \omega^{N-1-k}\right)=j_{*} \beta_{*}\left(\left(\operatorname{pr}_{1}^{*} \omega^{N-1-k}\right){ }_{\mid W_{Z}}\right)
$$

and $\beta_{*}\left(\left(\operatorname{pr}_{1}^{*} \omega^{N-1-k}\right)_{\mid W_{z}}\right)$ is of bidegree $\operatorname{dim} Z^{*}-k$, therefore $k \leq q-1$. On the other hand we have

$$
\int_{W_{Z}} \operatorname{pr}_{1}^{*} \omega^{N-1-k} \wedge \operatorname{pr}_{2}^{*}\left(\omega^{*}\right)^{k}=\int_{P(V)} \operatorname{pr}_{1 *}\left(\left[W_{Z}\right] \wedge \operatorname{pr}_{2}^{*}\left(\omega^{*}\right)^{k}\right) \wedge \omega^{N-1-k}
$$

and with $\gamma$ the restriction of $\mathrm{pr}_{1}$ to $W_{Z}$ we use the commutative diagram

$$
\begin{array}{ccc}
W_{Z} \stackrel{i_{0}}{\rightarrow} & P(V) \times P\left(V^{*}\right) \\
\gamma \downarrow & \text { prr }_{1} \\
Z \stackrel{j_{1}}{\rightarrow} & P(V)
\end{array}
$$

which allows to express

$$
\operatorname{pr}_{1 *}\left(\left[W_{Z}\right] \wedge \operatorname{pr}_{2}^{*}\left(\omega^{*}\right)^{k}\right)=j_{1 *} \gamma_{*}\left(\left(\operatorname{pr}_{2}^{*}\left(\omega^{*}\right)^{k}\right)_{\mid W_{Z}}\right) .
$$

Now $\gamma_{*}\left(\left(\operatorname{pr}_{2}^{*}\left(\omega^{*}\right)^{k}\right)_{\mid W_{Z}}\right)$ is of bidegree $k-(q-1)$, therefore $k \geq q-1$ thus $k=q-1$. We conclude that

$$
\left(\begin{array}{c}
N-1 \\
q-1
\end{array}\right)^{-1} \operatorname{deg} W_{Z}=\int_{W_{Z}} \operatorname{pr}_{1}^{*} \omega^{N-q} \wedge \operatorname{pr}_{2}^{*}\left(\omega^{*}\right)^{q-1}=\int_{Z} \omega^{N-q} \gamma_{*}\left(\left(\operatorname{pr}_{2}^{*}\left(\omega^{*}\right)^{q-1}\right)_{\mid W_{Z}}\right) .
$$

Since the fibers of $\gamma$ are projective subspaces of dimension $q-1$, we have

$$
\gamma_{*}\left(\left(\operatorname{pr}_{2}^{*}\left(\omega^{*}\right)^{q-1}\right)_{\mid W_{Z}}\right)=1
$$

thus the equalities $M \operatorname{deg} Z^{*}=\left(\begin{array}{c}N-1 \\ q-1\end{array}\right)^{-1} \operatorname{deg} W_{Z}=\operatorname{deg} Z$.

When $T=\lim _{\nu} Z_{\nu}$, then $W_{Z_{\nu}}$ has bounded degree by Proposition 9. Therefore we can assume that $W_{Z_{\nu}}$ converges to a closed positive current on $P(V) \times P\left(V^{*}\right)$, with support in the incidence manifold

$$
\left\{([x],[\lambda]) \in P(V) \times P\left(V^{*}\right) \text { such that } \lambda(x)=0\right\} .
$$

This current is a direct image of a closed positive current $W_{T}$ defined on this manifold.

Let us now prove that $W_{T}$ does not depend on the sequence $Z_{\nu}$. In effect, the map $W_{Z} \rightarrow Z^{*}$ is injective, since

$$
Z^{*}=0 \Rightarrow Z=0 \Rightarrow W_{Z}=0 .
$$

Thus there is a continuous linear operator $\mathcal{S}$ such that $W_{Z}=\mathcal{S}\left(Z^{*}\right)$, inverting the formula (ii) of Proposition 9. Since $Z_{\nu}^{*} \rightarrow T^{*}$ by Proposition 3, then $W_{Z_{\nu}}$ converges to $\mathcal{S}\left(T^{*}\right)$. So $W_{T}=\mathcal{S}\left(T^{*}\right)$ is independent of $Z_{\nu}$. 
Theorem 4. For all closed positive $(q, q)$-currents $T$ on $P(V)$, the conormal current $\operatorname{con}(T)$ is a closed positive current on $T^{*} P(V)$ defined by

$$
\operatorname{con}(T)=\alpha^{*}\left(W_{T}\right)
$$

and satisfying the inversion formula for the Chow transformation

$$
\operatorname{con}(T)=\Phi_{\star} \Psi^{*} \Psi_{\star} \Phi^{*}(\operatorname{con}(T)) .
$$

\subsection{Expression of $\mathcal{C} * \mathcal{C}$ from the Crofton formula}

For $u$ a smooth differential $(q, q)$-form on $P(V)$, we now calculate $\mathcal{C}^{*} \mathcal{C}(u)$ from formula (12) by expressing $\Omega^{d+q-1-j}$ thanks to a Crofton formula.

Recall that $d=(q-1)(N+1-q)=d_{G}-N-1+q$ with $d_{G}=\operatorname{dim} G(q, V)$, therefore $d+q-1-j=$ $d_{G}-(N+2+j-2 q)$. Since $j=\max (0,2 q-N-1)$, the bidimension of $\Omega^{d+q-1-j}$ is

$$
\begin{array}{ll}
(1,1) & \text { if } 2 q \geq N+1 \\
(N+2-2 q, N+2-2 q) & \text { if } 2 q \leq N+1
\end{array}
$$

and when $j \neq 0$ the cohomology class $\{\Omega\}^{d+q-1-j}=\{\Omega\}^{d_{G}-1}$ on $G(q, V)$ contains a multiple of a Schubert cycle $\sigma$ (see [12, 30]).

In effect, if $2 q \geq N+1$, let $V_{q-1} \subset V_{q+1}$ be vectorial subspaces of $V=\mathbb{C}^{N+1}$ of respective dimensions $q-1$ and $q+1$. Then we can take

$$
\sigma=\sigma_{V_{q-1}, V_{q+1}}=\left\{s \in G(q, V), V_{q-1} \subset s \subset V_{q+1}\right\}=P\left(V_{q+1} / V_{q-1}\right) \simeq \mathbb{P}_{1}
$$

because $V_{q-1} \subset s \subset V_{q+1}$ is equivalent to a system of homogeneous equations of degree 1 .

In this case the Crofton formula can be stated as

$$
\Omega^{d_{G}-1}=K \int_{V_{q-1} \subset V_{q+1}}[\sigma] \mathrm{d} \nu=K \int_{V_{q-1} \subset V_{q+1}}\left[\sigma_{V_{q-1}, V_{q+1}}\right] \mathrm{d} \nu\left(V_{q-1}, V_{q+1}\right)
$$

where $K$ is a constant factor and where $\nu$ is the measure on

$$
\left\{\left(V_{q-1}, V_{q+1}\right) \in G(q-1, V) \times G(q+1, V) \text { such that } V_{q-1} \subset V_{q+1}\right\}
$$

associated to the Hermitian metric induced by that of $G(q-1, V) \times G(q+1, V)$. For the proof, we note that

$$
\int_{V_{q-1} \subset V_{q+1}}[\sigma] \mathrm{d} \nu
$$

is invariant by the unitary group $U(N+1)=U(V)$, thus is harmonic. Since it is cohomologous on $G(q, V)$ to a multiple of $\Omega^{d_{G}-1}$, it is necessarily equal to a multiple of $\Omega^{d_{G}-1}$.

With $i: \sigma \rightarrow G(q, V)$ the canonical injection, we have the expected expression

$$
K^{-1} \mathcal{C}^{*} \mathcal{C}(u)=\int_{V_{q-1} \subset V_{q+1}} \varphi_{*} \psi^{*}\left([\sigma] \wedge \psi_{*} \varphi^{*} u\right) \mathrm{d} \nu=\int_{V_{q-1} \subset V_{q+1}} \varphi_{*} \psi^{*} i_{*} i^{*} \psi_{*} \varphi^{*} u \mathrm{~d} \nu
$$

where $\varphi_{*} \psi^{*} i_{*}$ is the transposed map of $i^{*} \psi_{*} \varphi^{*}$.

Now we replace $u$ by the integration current $[Z]$ associated to an effective algebraic cycle $Z$ of $P(V)$ of codimension $q$ and of degree $\operatorname{deg} Z$. First

$$
\mathcal{C}(Z)=\{s \in G(q, V), P(s) \cap Z \neq \varnothing\} \Rightarrow i^{*} \mathcal{C}(Z)=\left\{s \in G(q, V), V_{q-1} \subset s \subset V_{q+1} \text { and } P(s) \cap Z \neq \varnothing\right\} .
$$

We can assume that $P\left(V_{q+1}\right) \cap Z$ is a finite set of $\operatorname{deg} Z$ points $\left[x_{1}\right],\left[x_{2}\right], \ldots$ and so

$$
i^{*} \mathcal{C}(Z)=\left\{V_{q-1} \oplus \mathbb{C} x_{1}, V_{q-1} \oplus \mathbb{C} x_{2}, \ldots\right\} .
$$


As a consequence we obtain the formula

$$
\varphi_{\star} \psi^{*} i_{*} i^{*} \mathcal{C}(Z)=\sum_{1 \leq l \leq \operatorname{deg} Z}\left[P\left(V_{q-1} \oplus \mathbb{C} x_{l}\right)\right]
$$

which allows to reprove directly the injectivity of the map $Z \rightarrow \mathcal{C}^{*} \mathcal{C}([Z])$ obtained by the following result.

Proposition 10. For $Z$ an effective algebraic cycle of $P(V)$ of codimension $q$, the singular support of the current $\mathcal{C}^{*} \mathcal{C}([Z])$ is

$$
\operatorname{sing} \operatorname{supp}\left(\mathcal{C}^{*} \mathcal{C}([Z])\right)=\operatorname{supp} Z
$$

Proof. With $\Sigma=\mathcal{C}(Z)$ the Chow divisor of $Z$ and $\psi^{-1} \Sigma$ the inverse image divisor in $\Gamma$, we have

$$
\mathcal{C}^{*} \mathcal{C}([Z])=\varphi_{*} \psi^{*}\left(\Omega^{d+q-1-j} \wedge[\Sigma]\right)=\left(\varphi_{\mid \psi^{-1} \Sigma}\right)_{*}\left(\left(\psi^{*} \Omega^{d+q-1-j}\right)_{\mid \psi^{-1} \Sigma}\right) .
$$

For $[x] \in P(V)$, let us evaluate the dimension of the fiber $\left(\varphi_{\mid \psi^{-1} \Sigma}\right)^{-1}([x])=\varphi^{-1}([x]) \cap \psi^{-1} \Sigma$.

If $[x] \in Z$, then (see [25]) we have

$$
\varphi^{-1}([x]) \subset \psi^{-1} \Sigma .
$$

If $[x] \notin Z$, then $\varphi^{-1}([x]) \notin \psi^{-1} \Sigma$ and therefore $\varphi^{-1}([x]) \cap \psi^{-1} \Sigma$ is a divisor in $\varphi^{-1}([x])$ and is of constant dimension. As a consequence, $\mathcal{C}^{*} \mathcal{C}([Z])$ is smooth in $P(V) \backslash Z$.

On the other hand, by Theorem 2 we have $\mathcal{P} \mathcal{C}^{*} \mathcal{C}([Z])=[Z]$, thus $\mathcal{C}^{*} \mathcal{C}([Z])$ is not smooth near $[x]$ if $[x] \in Z$.

So $\mathcal{C}^{*} \mathcal{C}([Z])=\mathcal{C}^{*} \mathcal{C}\left(\left[Z^{\prime}\right]\right) \Rightarrow Z=Z^{\prime}$ when $Z$ and $Z^{\prime}$ are irreducible. Further there is a continuous linear operator $\mathcal{P}$ such that $[Z]=\left(\mathcal{P} \mathcal{C}^{*} \mathcal{C}\right)([Z])$ when $Z$ is irreducible. By linearity, this equality can be extended to each algebraic cycle $Z$.

In other words, with

$$
P\left(V_{q+1}\right) \cap Z^{\prime}=\left\{\left[x_{1}^{\prime}\right],\left[x_{2}^{\prime}\right], \ldots\right\},
$$

we have

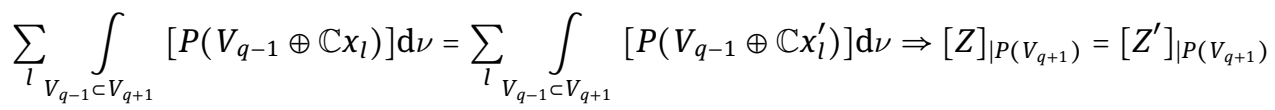

for all $V_{q+1} \in G(q+1, V)$ i.e. $[Z]=\left[Z^{\prime}\right]$.

If $2 q \leq N+1$, let $V_{q-1} \subset V_{N+2-q}$ be vectorial subspaces of $V=\mathbb{C}^{N+1}$ of respective dimensions $q-1$ and $N+2-q$. Then we can take the Schubert cycle

$$
\sigma=\sigma_{V_{q-1}, V_{N+2-q}}=\left\{s \in G(q, V), V_{q-1} \subset s \subset V_{N+2-q}\right\}=P\left(V_{N+2-q} / V_{q-1}\right) \simeq \mathbb{P}_{N+2-2 q} .
$$

In that case the Crofton formula can be stated as

$$
\mathcal{B}=\int_{V_{q-1} \subset V_{N+2-q}}[\sigma] \mathrm{d} \nu=\int_{V_{q-1} \subset V_{N+2-q}}\left[\sigma_{V_{q-1}, V_{N+2-q}}\right] \mathrm{d} \nu\left(V_{q-1}, V_{N+2-q}\right)
$$

where $\mathcal{B}$ is a harmonic differential form of bidegree $\left(d_{G}-N-2+2 q, d_{G}-N-2+2 q\right)$ and where $\nu$ is the measure on

$$
\left\{\left(V_{q-1}, V_{N+2-q}\right) \in G(q-1, V) \times G(N+2-q, V) \text { such that } V_{q-1} \subset V_{N+2-q}\right\}
$$

associated to the Hermitian metric induced by that of $G(q-1, V) \times G(N+2-q, V)$.

With $i: \sigma \rightarrow G(q, V)$ the canonical injection, when we replace $u$ by $[Z]$ in the transform

$$
\int_{V_{q-1} \subset V_{N+2-q}} \varphi_{*} \psi^{*} i_{*} i^{*} \psi_{*} \varphi^{*} u \mathrm{~d} \nu
$$

of $u$, we use that

$$
i^{*} \mathcal{C}(Z)=\left\{s \in G(q, V), V_{q-1} \subset s \subset V_{N+2-q} \text { and } P(s) \cap Z \neq \varnothing\right\}
$$


and we still denote by $\pi: V \backslash\{0\} \rightarrow P(V)$ the canonical map. We extend $\pi^{-1}(Z)$ as an analytic subset of $V$. Then for $s \in G(q, V)$, we have $s \in \operatorname{supp} i^{*} \mathcal{C}(Z)$ if and only if $s=V_{q-1} \oplus \mathbb{C} x$ with

$$
x \in\left(V_{q-1}+\pi^{-1}(Z)\right) \cap\left(V_{N+2-q} \backslash V_{q-1}\right) .
$$

As a consequence, we obtain that $\varphi_{*} \psi^{*} i_{*} i^{*} \mathcal{C}(Z)$ is the image by $\pi$ of

$$
\left(V_{q-1}+\left(\left(V_{q-1}+\pi^{-1}(Z)\right) \cap V_{N+2-q}\right)\right) \backslash\{0\} .
$$

The injectivity of the previous transform means that if

$$
\left(V_{q-1}+\pi^{-1}(Z)\right) \cap V_{N+2-q}=\left(V_{q-1}+\pi^{-1}\left(Z^{\prime}\right)\right) \cap V_{N+2-q}
$$

for all $V_{q-1} \subset V_{N+2-q}$, then $Z=Z^{\prime}$.

\subsection{Skoda potential of a closed positive current}

For $T$ a closed positive $(q, q)$-current on $\mathbb{P}_{N}=P(V)$, we now express the Skoda potential $U$ associated to $T$ (see $[5,28,29]$ ) by means of the Chow transform $\mathcal{C}(T)$. This function is quasi plurisubharmonic on $\mathbb{P}_{N}$ and

$$
(\operatorname{deg} T) \omega+\operatorname{dd}^{c} U
$$

is obtained from $\mathcal{C}(T)$ with an integral transform. Precisely we set

$$
T_{1}=\varphi_{\star} \psi^{*}(\mathcal{A} \wedge \mathcal{C}(T))=\varphi_{*} \psi^{*}\left(\mathcal{A} \wedge \psi_{*} \varphi^{*} T\right)
$$

where $\mathcal{A}$ is a positive harmonic differential $(d, d)$-form on $G(q, V)$. Then $T_{1}$ is a closed positive $(1,1)$-current on $\mathbb{P}_{N}$ and

$$
T_{1}=\operatorname{pr}_{1 *}\left(K \wedge \operatorname{pr}_{2}^{*} T\right)
$$

where the integral kernel $K$ on $\mathbb{P}_{N} \times \mathbb{P}_{N}$ is given as in Proposition 5 by

$$
K=q_{*}\left(\left(\psi \circ p_{1}\right)^{*} \mathcal{A}\right) .
$$

We express $\mathcal{A}$ thanks to a Crofton formula. First for $V_{q-1} \in G(q-1, V)$ we consider the Schubert cycle $\sigma$ defined by

$$
\sigma=\sigma_{V_{q-1}}=\left\{s \in G(q, V), V_{q-1} \subset s\right\}=P\left(V / V_{q-1}\right) \simeq \mathbb{P}_{p+1} .
$$

The condition $V_{q-1} \subset s$ is equivalent to a system of homogeneous equations of degree 1 . The Crofton formula gives that

$$
\mathcal{A}=\int_{V_{q-1} \in G(q-1, V)}[\sigma] \mathrm{d} \nu=\int_{V_{q-1} \in G(q-1, V)}\left[\sigma_{V_{q-1}}\right] \mathrm{d} \nu\left(V_{q-1}\right)
$$

is harmonic, where $\nu$ is the positive measure on $G(q-1, V)$ which is invariant by $U(N+1)$ and of total mass equal to 1 . As a consequence

$$
K=\int_{V_{q-1} \in G(q-1, V)} q_{*}\left(\left(\psi \circ p_{1}\right)^{*}[\sigma]\right) \mathrm{d} \nu
$$

with

$$
\begin{aligned}
q_{*}\left(\left(\psi \circ p_{1}\right)^{*} \sigma\right) & =q\left(\left\{\left([x],\left[x^{\prime}\right], s\right) \text { such that } x \in s, x^{\prime} \in s, V_{q-1} \subset s\right\}\right) \\
& =\left\{\left([x],\left[x^{\prime}\right]\right) \text { such that } \mathbb{C} x \oplus V_{q-1}=\mathbb{C} x^{\prime} \oplus V_{q-1}\right\} .
\end{aligned}
$$

Let $\pi_{V_{q-1}}: P(V) \backslash P\left(V_{q-1}\right) \rightarrow P\left(V_{q-1}^{\perp}\right)$ be the projective map associated to the orthogonal projection $V \rightarrow V_{q-1}^{\perp}$. Then

$$
q_{*}\left(\left(\psi \circ p_{1}\right)^{*} \sigma\right)=\left(\pi_{V_{q-1}} \times \pi_{V_{q-1}}\right)^{*} D_{P\left(V_{q-1}^{\perp}\right)}
$$


where $D_{P\left(V_{q-1}^{\perp}\right)}$ is the diagonal of $P\left(V_{q-1}^{\perp}\right) \times P\left(V_{q-1}^{\perp}\right)$. In other words the integral kernel is

$$
K=\int_{V_{q-1} \in G(q-1, V)}\left(\pi_{V_{q-1}} \times \pi_{V_{q-1}}\right)^{*}\left[D_{P\left(V_{q-1}^{\perp}\right)}\right] \mathrm{d} \nu
$$

and we know it is enough to prove the following result.

Theorem 5. The closed positive $(1,1)$-current $T_{1}$ on $\mathbb{P}_{N}$ satisfies $T_{1}=(\operatorname{deg} T) \omega+\operatorname{dd}^{\mathrm{c}} U$ with $U$ the Skoda potential associated to $T$. Thus we have the equalities:

(i) $\operatorname{deg} T_{1}=\operatorname{deg} T$ for the degrees with respect to $\omega$,

(ii) $\nu\left(T_{1},[x]\right)=\nu(T,[x])$ for the Lelong numbers at each point $[x] \in \mathbb{P}_{N}$.

\section{Chow transform defined on the space of cycles}

Let $X$ be a complex projective manifold of dimension $d_{X}$ and let $T$ be a smooth differential form on $X$ of bidimension $(p, p)$ with $p=d_{X}-q$. The Chow transform $\hat{\mathcal{C}}(T)$ of $T$ is a current of bidegree $(1,1)$ on the space $C_{q-1}(X)$ of effective algebraic cycles in $X$ of dimension $q-1$, obtained in the following way.

Let $\hat{\Gamma}$ be the incidence variety i.e. the subvariety in $X \times C_{q-1}(X)$ of $(x, c)$ satisfying $c \ni x$. With $\hat{\varphi}: \hat{\Gamma} \rightarrow X$ and $\hat{\psi}: \hat{\Gamma} \rightarrow C_{q-1}(X)$ the restrictions to $\hat{\Gamma}$ of the canonical projections, we set

$$
\hat{\mathcal{C}}(T)=\hat{\psi}_{*} \hat{\varphi}^{*} T .
$$

The differential form $\hat{\mathcal{C}}(T)$ can be calculated by integration along the fibers of $\hat{\psi}$. For $c \in C_{q-1}(X)$ and $\sigma \in$ $T_{c} C_{q-1}(X)$ a tangent vector, we have

$$
\hat{\mathcal{C}}(T)_{c}(\sigma, \bar{\sigma})=\int_{x \in c} T_{x}\left(\xi_{x}, \bar{\xi}_{x}, \ldots\right)
$$

where $\xi_{x} \in T_{x} X$ is the projection of a lifting of $\sigma$ by $\mathrm{d} \hat{\psi}_{(x, c)}$ i.e. $\xi_{x}$ satisfies $\left(\xi_{x}, \sigma\right) \in T_{(x, c)} \hat{\Gamma}$. We denote by $N_{X} c$ the normal bundle to $c$ in $X$ and we use the linear map (see [4, 10, 23])

$$
T_{c} C_{q-1}(X) \rightarrow H^{0}\left(c, N_{X} c\right)
$$

which associates to $\sigma$ the infinitesimal moving $\tilde{\sigma}$. Then the condition for $\xi_{x}$ is

$$
\tilde{\sigma}(x)=-\xi_{x} \bmod T_{x} c \in T_{x} X / T_{x} c
$$

for all $x \in c$.

\subsection{A commutative diagram}

We consider $j: X \rightarrow P(V)$ an embedding of $X$ into $\mathbb{P}_{N}=P(V)$ and the induced meromorphic map

$$
\rho: G\left(p+1, V^{*}\right) \rightarrow C_{q-1}(X)
$$

which associates $X \cap P\left(\operatorname{ker} \lambda_{0}\right) \cap \ldots \cap P\left(\operatorname{ker} \lambda_{p}\right)$ to vect $\left(\lambda_{0}, \ldots, \lambda_{p}\right) \in G\left(p+1, V^{*}\right)$.

Lemma 8. For $T$ a smooth differential form of bidimension $(p, p)$ on $X$, we have the equality $\rho^{*} \hat{\mathcal{C}}(T)=\mathcal{C}\left(j_{*} T\right)$ between $(1,1)$-currents on $G\left(p+1, V^{*}\right)=G(N-p, V)$.

Proof. This is equivalent to

$$
\left\langle\hat{\mathcal{C}}(T), \rho_{\star} v\right\rangle=\left\langle\mathcal{C}\left(j_{\star} T\right), v\right\rangle \Leftrightarrow\left\langle T, \hat{\varphi}_{*} \hat{\psi}^{*} \rho_{\star} v\right\rangle=\left\langle T,\left(\varphi_{*} \psi^{*} v\right)_{\mid X}\right\rangle
$$


for each smooth differential form $v$ of bidimension $(1,1)$ on $G\left(p+1, V^{*}\right)$. With $r(x, s)=(x, X \cap P(s))$, the diagram

$$
\begin{aligned}
\Gamma \cap(X \times G(N-p, V)) & \stackrel{r}{\rightarrow} \hat{\Gamma} \\
\psi \downarrow & \\
G(N-p, V) & \stackrel{\rho}{\rightarrow} C_{q-1}(X)
\end{aligned}
$$

is commutative. Therefore

$$
\hat{\varphi}_{*} \hat{\psi}^{*} \rho_{*} v=\hat{\varphi}_{*} r_{*}\left(\left(\psi^{*} v\right)_{\mid \varphi^{-1}(X)}\right)=\left(\varphi_{\mid \varphi^{-1}(X)}\right)_{*}\left(\left(\psi^{*} v\right)_{\mid \varphi^{-1}(X)}\right)=\left(\varphi_{*} \psi^{*} v\right)_{\mid X} .
$$

Proposition 11. The transformation of integration along the fibers

$$
\hat{\mathcal{C}}:\{\text { smooth differential }(q, q) \text {-forms on } X\} \rightarrow\left\{(1,1) \text {-currents on } C_{q-1}(X)\right\}
$$

is injective.

Proof. This a consequence of Lemma 8 and of the injectivity of $\mathcal{C}$.

Remark 2. The transformation $\hat{\mathcal{C}}$ can be used to solve the problem of approximating closed $(q, q)$-currents by algebraic cycles.

In effect, if $T$ is closed, $\hat{\mathcal{C}}(T)$ is a closed $(1,1)$-current on $C_{q-1}(X)$ of order 0 and if moreover $\{T\}$ is rational, $\hat{\mathcal{C}}(T)$ is a weak limit of divisors with complex coefficients. Then we can write

$$
\hat{\mathcal{C}}(T)=\int_{D \in \operatorname{Div}} \lambda(D)[D]
$$

where Div $C_{q-1}(X)$ is the space of divisors of $C_{q-1}(X)$ and $\lambda$ is a measure on Div $C_{q-1}(X)$. The LebesgueNikodym decomposition of $\lambda$ is

$$
\lambda=\lambda_{0}+\lambda_{1}
$$

with $\lambda_{0}$ a $L_{\text {loc }}^{1}$ function and $\lambda_{1}$ a measure such that supp $\lambda_{1}$ is negligible. We now determine supp $\lambda_{1}$ by using the injection $C_{p}(X) \hookrightarrow \operatorname{Div} C_{q-1}(X)$ which associates to $Z \in C_{p}(X)$ the incidence divisor

$$
D_{Z}=\left\{c \in C_{q-1}(X), Z \cap c \neq \varnothing\right\}
$$

of $Z$. We fix $D_{0} \in \operatorname{Div} C_{q-1}(X)$ and we consider $w$ a current of bidimension $(1,1)$ on $C_{q-1}(X)$ such that $D \rightarrow$ $\int_{D} w$ is the Dirac measure at $D_{0}$. Thus

$$
\left\langle T, \hat{\varphi}_{*} \hat{\psi}^{*} w\right\rangle=\int_{D \in \operatorname{Div}} \lambda(D) \int_{D} w
$$

is a value of $\int_{\operatorname{Div} C_{q-1}(X)} \lambda \delta_{D_{0}}$ in a generalized sense. We set

$$
Z_{0}=\left\{x \in X, \hat{\varphi}^{-1}(x) \subset \hat{\psi}^{-1}\left(D_{0}\right)\right\}=\left\{x \in X, c \ni x \Rightarrow c \in D_{0}\right\} \subset X
$$

which is of dimension $\leq p$ and satisfies $\operatorname{dim} Z_{0}=p$ when $D_{0}$ is an incidence divisor.

Assume $D_{0} \notin C_{p}(X)$ i.e. $\operatorname{dim} Z_{0}<p$, then the singularities of the current $\hat{\varphi}_{*} \hat{\psi}^{*} w$ are of a lower order and $\int_{\text {Div } C_{q-1}(X)} \lambda \delta_{D_{0}}$ is the effective value of $\lambda$ at $D_{0}$. In other words $D_{0} \notin \operatorname{supp} \lambda_{1}$.

Conclusion : supp $\lambda_{1} \subset C_{p}(X)$, which is enough to prove that $T$ is a weak limit of algebraic cycles with complex coefficients.

\subsection{A kinematic formula}

Here we retrieve the equations (8) and (9), which characterize the Chow transform of a smooth differential $(q-1, q-1)$-form $u$ on $P(V)=\mathbb{P}_{N}$, by using the following kinematic formula (see [15])

$$
\frac{\mathrm{d}}{\mathrm{d} t}\left(\int_{c_{t}} u\right)_{\mid t=0}=\frac{\mathrm{d}}{\mathrm{d} t}\left(\left(\hat{\psi}_{*} \hat{\varphi}^{*} u\right)\left(c_{t}\right)\right)_{\mid t=0}=\left(\hat{\psi}_{*} \hat{\varphi}^{*} \mathrm{~d} u\right)_{c}\left(\frac{\mathrm{d} c_{t}}{\mathrm{~d} t}{ }_{\mid t=0}\right)
$$


where $c=c_{0}$ with $c_{t} \in C_{q-1}\left(\mathbb{P}_{N}\right)$ an one-parameter family of effective algebraic cycles of $\mathbb{P}_{N}$ of dimension $q-1$, which is differentiable at $t=0$.

In particular, we consider the family of projective subspaces of $\mathbb{P}_{N}=P(V)$ of dimension $q-1$, which is parametrized by $G(q, V)=\mathbb{G}_{q-1, N}$. We use homogeneous coordinates on $G(q, V)$ i.e. we write $s \in G(q, V)$ as being

$$
s=\tau(z)=\operatorname{vect}\left(z^{0}, \ldots, z^{q-1}\right)
$$

for $z=\left(z^{0}, \ldots, z^{q-1}\right) \in V^{q}$. The associated Plücker coordinates are defined by

$$
z^{0} \wedge \ldots \wedge z^{q-1}=\sum_{|I|=q} z_{I} e_{I} \in \bigwedge^{q} V
$$

where $e_{I}=e_{i_{1}} \wedge \ldots \wedge e_{i_{q}}$ for $I=\left(i_{1}, \ldots, i_{q}\right)$ with $0 \leq i_{1}<\ldots<i_{q} \leq N$, denoting by $e_{0}, \ldots, e_{N}$ an orthonormal basis of $V$.

We define the differential operator in the homogeneous coordinates

$$
\partial_{I}=\operatorname{det}\left(\frac{\partial}{\partial z_{j}^{k}}\right)_{\substack{0 \leq k \leq q-1 \\ j \in I}}=\frac{\partial}{\partial z_{I}}
$$

and for $|I|=|J|=q$, we evaluate $\partial_{I} \bar{\partial}_{J} U$ where

$$
U(z)=\int_{P(\tau(z))} u
$$

by means of formula (29). In effect, first we have

$$
\left(\partial_{I} \bar{\partial}_{J} U\right)(z)=\left(\psi_{*} \varphi^{*}(\partial \bar{\partial} u)\right)_{s}\left(\frac{\partial s}{\partial z_{I}}, \frac{\overline{\partial s}}{\partial z_{J}}\right) .
$$

We introduce $i: G(q, V) \rightarrow P\left(\wedge^{q} V\right)$ the Plücker embedding, which allows to calculate

$$
\operatorname{di} i_{s}\left(\frac{\partial s}{\partial z_{I}}\right)=\frac{\partial}{\partial z_{I}}(i(s))=\left(z^{0} \wedge \ldots \wedge z^{q-1}\right)^{-1} \otimes\left(e_{I} \bmod \mathbb{C}\left(z^{0} \wedge \ldots \wedge z^{q-1}\right)\right) \in T_{\left[z^{0} \wedge \ldots \wedge z^{q-1}\right]} P(\stackrel{q}{\wedge} V)
$$

by means of (30). We set $v=\partial \bar{\partial} u$ and we use the previous formula for the Chow transform

$$
\tau^{*} \mathcal{C}(v)=\sum_{|I|=|J|=q} \tilde{v}_{I J}(z) \mathrm{d} z_{I} \wedge \mathrm{d} \bar{z}_{J}
$$

where $\tilde{v}_{I J}(z)$ is given by (14). Since the inverse image in $\wedge^{q} V$ of $\operatorname{di} i_{s}\left(\frac{\partial s}{\partial z_{I}}\right)$ is constant equal to $e_{I}$, the function $\partial_{I} \bar{\partial}_{J} U$ decompose on the $\tilde{v}_{I J}$ with constant coefficients. Therefore $\partial_{I} \bar{\partial}_{J} U$ is itself of the form $\tilde{v}$. But we know that such functions satisfy the John differential equations (see the proof of Theorem 3 for example)

$$
0=\left(\frac{\partial^{2}}{\partial z_{m}^{l} \partial z_{m^{\prime}}^{l^{\prime}}}-\frac{\partial^{2}}{\partial z_{m^{\prime}}^{l} \partial z_{m}^{l^{\prime}}}\right) \tilde{v} \text { and } 0=\left(\frac{\partial^{2}}{\partial \bar{z}_{m}^{l} \partial \bar{z}_{m^{\prime}}^{l^{\prime}}}-\frac{\partial^{2}}{\partial \bar{z}_{m^{\prime}}^{l} \partial \bar{z}_{m}^{l^{\prime}}}\right) \tilde{v}
$$

for all $0 \leq l, l^{\prime} \leq q-1$ and all $0 \leq m, m^{\prime} \leq N$.

As a conclusion, we find again that $U$ satisfies the differential equations

$$
0=\left(\frac{\partial^{2}}{\partial z_{m}^{l} \partial z_{m^{\prime}}^{l^{\prime}}}-\frac{\partial^{2}}{\partial z_{m^{\prime}}^{l} \partial z_{m}^{l^{\prime}}}\right) \partial_{I} \bar{\partial}_{J} U \text { and } 0=\left(\frac{\partial^{2}}{\partial \bar{z}_{m}^{l} \partial \bar{z}_{m^{\prime}}^{l^{\prime}}}-\frac{\partial^{2}}{\partial \bar{z}_{m^{\prime}}^{l} \partial \bar{z}_{m}^{l^{\prime}}}\right) \partial_{I} \bar{\partial}_{J} U
$$

for all $|I|=|J|=q$, all $0 \leq l, l^{\prime} \leq q-1$ and all $0 \leq m, m^{\prime} \leq N$. 


\section{References}

[1] Ben Messaoud H., El Mir H., Opérateur de Monge-Ampère et tranchage des courants positifs fermés, J. Geom. Anal. 10 (2000), no. 1, 139-168.

[2] Blel M., Sur le cône tangent à un courant positiffermé, J. Math. Pures Appl. (9) 72 (1993) 517-536.

[3] Blel M., Demailly J.-P., Mouzali M., Sur l'existence du cône tangent à un courant positif fermé, Ark. Mat. 28 (1990), no. 2, 231-248.

[4] Campana F., Peternell T., Cycle spaces, Encyclopaedia Math. Sci. 74, Springer, 1994, 319-349.

[5] Demailly J.-P., Monge-Ampère operators, Lelong numbers and intersection theory, Complex analysis and geometry, Univ. Ser. Math., Plenum Press, 1993, 115-193.

[6] Demailly J.-P., Analytic methods in algebraic geometry, Surveys of Modern Mathematics 1, International Press, 2012.

[7] Federer H., Geometric measure theory, Grundlehren der mathematischen Wissenschaften 153, Springer Verlag, 1969.

[8] Gelfand I.M., Gindikin S.G., Graev M.I., Integral geometry in affine and projective spaces, J. Soviet Math. 18 (1980) 39-167.

[9] Gelfand I.M., Graev M.I., Shapiro Z.Y., Differential forms and integral geometry, Functional Anal. Appl. 3 (1969) 101-114.

[10] Gelfand I.M., Kapranov M.M., Zelevinsky A.V., Discriminants,resultants and multidimensional determinants, Mathematics : Theory and Applications, Birkhäuser, 1994.

[11] Gindikin S.G., Michor P., 75 years of Radon transform, Conference Proceedings and Lecture Notes in Mathematical Physics IV, International Press, 1994.

[12] Griffiths P., Harris J., Principles of algebraic geometry, John Wiley \& Sons, 1978.

[13] Griffiths P., Schmid W., Locally homogeneous complex manifolds, Acta Math. 123 (1969) 253-302.

[14] Grinberg E.L., On images of Radon transforms, Duke Math. J. 52 (1985) 939-972.

[15] Grinberg E.L., Quinto E.T., Integral geometry and tomography, Contemp. Math. 113, 1990.

[16] Grothendieck A., Eléments de géométrie algébrique ch. IV, partie III Etude locale des schémas et des morphismes de schémas, Inst. Hautes Etudes Sci. Publ. Math. 28, 1966.

[17] Guillemin V., Perspectives in integral geometry, Integral geometry, Contemp. Math. 63, 1987, 135-150.

[18] Guillemin V., Sternberg S., Some problems in integral geometry and some related problems in microlocal analysis, Amer. J. Math. 101 (1979) 915-955.

[19] Helgason S., Differential geometry and symmetric spaces, Pure and Applied Mathematics, vol. XII, Academic Press, 1962.

[20] Helgason S., The Radon transform on Euclidean spaces, compact two-point homogeneous spaces and Grassmann manifolds, Acta Math. 113 (1965) 153-180.

[21] Helgason S., The Radon transform, Progress in Mathematics 5, Birkhäuser, 1980.

[22] Kiselman C.O., Tangents of plurisubharmonic functions, International Symposium in Memory of Hua Loo Keng vol. II, Springer, 1991, 157-167.

[23] Kodaira K., Complex manifolds and deformation of complex structures, Grundlehren der mathematischen Wissenschaften 283, Springer Verlag, 1986.

[24] Méo M., Transformation de Chow des courants définis dans une variété projective, C. R. Acad. Sci. Paris Série I 315 (1992) 1041-1044.

[25] Méo M., Caractérisation des courants associés aux cycles algébriques par leur transformé de Chow, J. Math. Pures Appl. 79 (2000) 21-56.

[26] Poly J.-B., Sur l'homologie des courants à support dans un ensemble semi-analytique, Bull. Soc. Math. France Suppl. Mém. 38 (1974) 35-43.

[27] Raby G., Tranchage des courants positifs fermés et équation de Lelong-Poincaré, J. Math. Pures Appl. (9) 75 (1996), no. 3 , 189-209.

[28] Skoda H., Sous-ensembles analytiques d'ordre fini ou infini dans $\mathbb{C}^{n}$, Bull. Soc. Math. France 100 (1972) 353-408.

[29] Skoda H., Nouvelle méthode pour l'étude des potentiels associés aux ensembles analytiques, Séminaire Lelong 1972-1973, Lecture Notes in Math. 410, 1974, 117-141.

[30] Stoll W., Invariant forms on Grassmann manifolds, Annals of Mathematics Studies 89, 1977. 